\title{
Compatibilidade de
}

Bxtensóes de Matroides

\section{Denise Salai}

DISSERTACÃO APRESENTA DA $\triangle O$

INSTITUTO DE MATEMATICA E ESTATISTICA D A

UNIVERSIDADE DE SÃO PAULO

PARA OBTENCÃO DO GRAU DE MESTRE BM

MATEM $\mathbb{T}$ TICA APLICADA

Área de Concentração: Ciência da Computação Orientador: Prof. Dr. Arnaldo Mandel

-SÃO PAULO, Junho de 1987- 

A minho fomelia 



\section{Agradecimentos}

A gente quer ser original, mas acaba tendo que agradecer à mesmas pessoas pelas mesmas coisas de sempre:

A minha familia, já agradeci.

Gostaria de fager um agradecimento especial a cada amigo, mas acho que o espaço não vai dar... Mesmo assim, obrigada a todos pelo apoio.

E para terminar, nem preciso diser que esse trabalho não teria sido realisado sem a ajuda do meu orientador. Arnaldo, obrigada por tudo! 



\section{Abstract}

It is generally dificult to characterime wich families of extensions of a matroid are compatible. Even when we restrict the problem to single-element extensions, no complete satisfactory answer is known. The object of this work is to study the problem of compatibility of single-element extensions of a matroid in two particular cases which are well understood.

In the first case, a natural sufficient condition, due to Cordovil [Co2], for a fnite family of single-element extensions to be compatible is given. By making use of the one-to-one correspondence between single-element extensions and modular filters of a matroid, first established by Crapo in $[\mathrm{Cr}$, Cordovil introduced the concept of ultra-compatibility of a family of modular flters that implies the compatibility of the single-element extensions associated with them. We give a further simple characterisation for the ultra-compatibility.

In the second case, we examine the problem of compatibility of a pair of singleelement extensions of a matroid. Good solutions for this problem are already Inown. We present several characterisations for compatible pairs of single-element extensions, whose authors developed different theories and techniques to solve this problem:

- Las Vergnas in [LV1] compared the filters associated with the two extensions and worked onf conditions in terns of their relative positions.

- Cordovil in [Co2] used a generalisation of the concept of modular filter (gerbe) introduced by Las Verguas in [LV2].

- Cheung in $[\mathrm{Ch}]$ obtained three distinct solutions based, respectively, on the notion of closure of a modular iner, on linear subclasses and on the notion of quotient buadle. 



\section{Introdução}

A Teoria dos Matróides teve sua origem na tentativa de van der Waerden [vW] de axiomatisar e generalizar os conceitos de dependência linear e algébrica no ińcio da década de 1930. A essa época, Whitney [Wh] percebeu que é possivel associar às árvores de um grafo um conceito de dependência semelhante. Como resultado dessas observaçōes, surgiu em 1935 um artigo que lançou os fundamentos da teoria em uma forma que pouco mudou até hoje. Com a descoberta gradual de novas classes de matróides, verificon-se que essa teoria consegue unificar também vários conceitos da Teoria dos Grafos, Reticulados, Trangversais e Estruturas de Incidência. É justamente nessa troca de idéias com diferentes ramos da Combinatória que reside um dos principais e um dos mais úteis aspectos da Teoria dos Matróides.

Devido ao seu caráter geométrico, os espaços projetivos e afins são ferramentas de fundamental importância no estudo da Combinatória. Muitos dos problemas relacionados com matróides também tiveram origem dentro desse contexto. Tentaremos através desses espaços, introdusir alguns conceitos básicos da Teoria dos Matróides, ben como apresentar algumas questões ligadas com a Compatibilidade de Extensōes de Matróides, principal objeto de investigação deste trabalho.

No que se segue, é dada uma configuração $C$ de pontos num espaço (projetivo ou afim) de dimensão finita, isto $\varepsilon$, am conjunto finito $E$ de pontos nesse espaço. Nos exemplos, vamos explicitar apenas as linhas com mais de três pontos 
e, em certos casos, explicitaremos também alguns planos para uma melhor visualisação. Abaixo, apresentamos dois exemplos bem conhecidos de configurações de pontos nos espaços projetivos de dimensão 2 e 3 respectivamente:

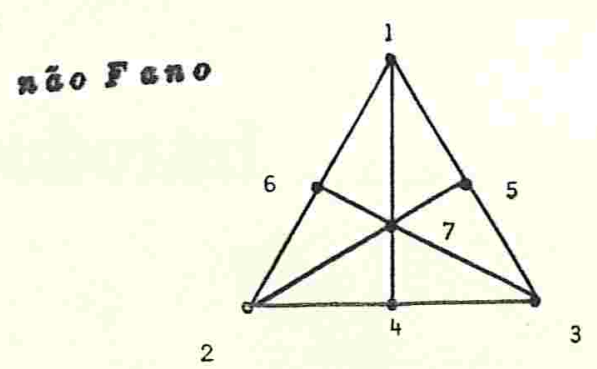

fig.1.

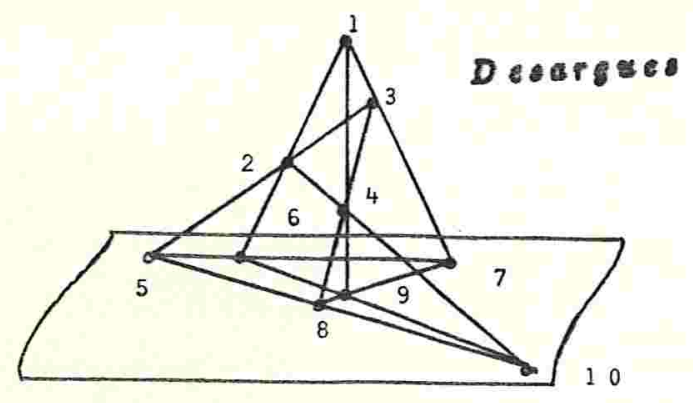

fig.1.b

A intersecção de um subespaço com a configuração $C$ aerá chamada de fechado de $C$. Observe que com essa definição, $E$ é um fechado e a interseç̧ão de dois fechados também é um fechado. Assim, a famllia dos fechados da configuração $C$ quando ordenados por inclusão forma um reticulado. Abaixo temos por exemplo, o reticulado dos fechados da contiguração da fig.1.a.

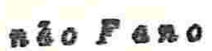

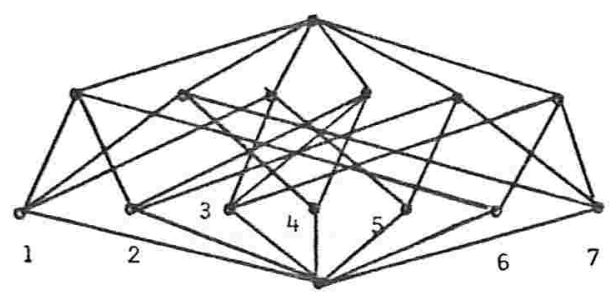

fig.2

Assocjamos à cada subconjunto $X$ de $E$ o natural $\operatorname{dim}(X)+1$, onde $\operatorname{dim}(X)$ é a dimensão do subespaço gerado por $X$. Esse natural será chamado de posto de $X$ em $C$, denotado por $\rho \mathcal{C}(\boldsymbol{X})$. A função $\rho C$ tem duas propriedades óbvias:

i. $0 \leq \rho c(X) \leq|X|$ para todo $X \subseteq E$

ii. Se $X \subseteq Y \subseteq E$ então $\rho c(X) \leq \rho c(Y)$

Voltemos a fixar nossa atenção no reticulado dos fechados de C. A função $\rho c$ quando restrita a esses fechados é na realidade uma função altura para o reticulado. Um resultado bem conhecido sobre espaços projetivos dis que

$$
\operatorname{dim}(\boldsymbol{X})+\operatorname{dim}(\boldsymbol{Y})=\operatorname{dim}(\boldsymbol{X} \cup \boldsymbol{Y})+\operatorname{dim}(\boldsymbol{X} \cap \boldsymbol{Y})
$$

para quaisquer aubespaços $X$ e $Y$. Não se espera que a igualdade acima permaneçs válida quando $X$ e $Y$ são fechados de $\mathcal{C}, j a ́$ que nesse caso $X$ e $Y$ az̃o apenas 
intersecções de subespaços com a conf́guração. Entretanto, ainda assim, vale uma condição mais fraca, a submodularidade de $\rho$ c sobre os fechados, isto é,

$$
\rho c(X)+\rho_{c}(Y) \geq \rho c(X \cup Y)+\rho c(X \cap Y)
$$

para quaisquer fechados $X$ e $Y$. Um reticulado com funçz̃o altura finita e submodular onde todo elemento pode ser expresso como supremo de átomos es chamado reticalado geométrico.

Toda essa discussão envolvendo fechados, posto reticulado geométrico vale para matróides em geral. Aliás, a partir de cada um desses conceitos, podemos obter uma definição axiomática de matróides. Em particular, um matróide $M$ pode ser definido como sendo um conjunto finito $E$ juntamente com uma famflia de subconjuntos de $E$, chamados fechados do matroide, com propriedades semelhantes àquelas dos fechados da configuração C. Definiçōes precisas encontram "se entre as Preliminares mas não são necessárias para acompanhar a discussão introdutória.

O raciocínio feito sobre a conf́guração $C$ continua valendo para qualquer subconjunto de pontos de $\boldsymbol{E}$. O correspondente a essa idéia para matróides em geral é dado pela noção de submatróide. Um submatroide de um matróide $M$ sobre um conjunto $E$ é um matróide sobre um subconjunto $A$ de $E$, chamado de restriçōo de $M$ a $A$ e denotado por $M(A)$, cujos fechados são as interseç̧öes entre os fechados de $M$ e o conjunto $A$. Chegamos finalmente no ponto de definir um dos principais conceitos presentes nesse trabalho. Uma estensäo $N$ do matróide $M$ é um matróide que tem $M$ como submatróide. Como ilustração, vamos examinar as extensões da configuração de pontos $C$ obtidas simplesmente pelo acréscimo de novos pontos. Note que todas essas extensões podem ser obtidas acrescentando-se um novo ponto por vez e por essa rasão vamos nos restringir apenas às extensões pontuais da configuração. Para fixar idéias, suponha que $C^{\prime}$ seja a configuração de 4 pontos no plano, $\{a, b, c, d\}$, na qual não existem 3 pontos colineares. A presentamos a seguir, $s$ extensōes pontuais de $C^{\prime}$ por um novo ponto $p$.

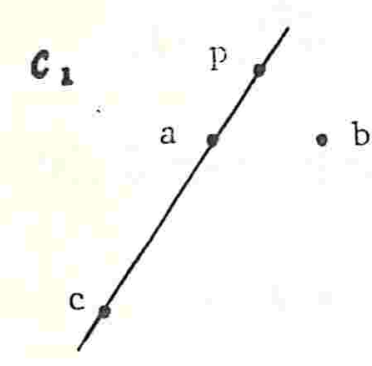

fig.3.6

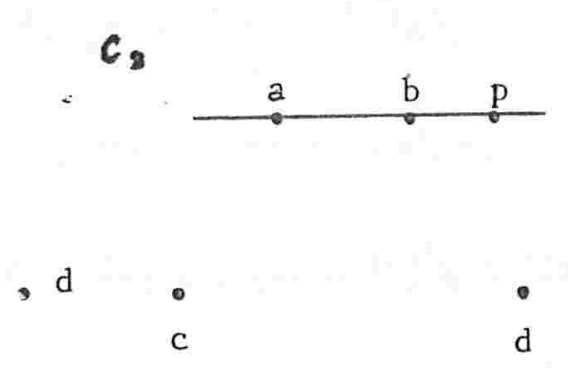

fig.3.b

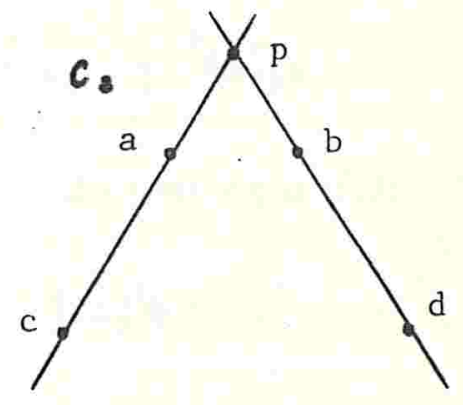

fig.3.e

Observe que diferentes extensões de $C^{\prime}$ são determinadas por diferentes posições do ponto $p$ em relação a 0 fechados de $C^{\prime}$. Antes de analisarmos a estrutura 
dos fechados das extensões desse exemplo com mais detalhe, introdngiremos outro conceito importante.

O fecho de $X \subseteq E$ da configuração de pontos $C$, denotado por $\bar{X}^{C}$, \& o menor fechado de $C$ que contém $X$, ou equivalentemente, é a intersecção de todos os fechados de $C$ que têm $X$ como aubconjunto. Não é difĺcil verificar que:

i. Para todo $X \subseteq E$ temos $X \subseteq \bar{X}^{C}$ e a igualdade ocorre se somente se $X$ é fechado de $\mathcal{C}$

ii. Para todo $X \subseteq E$ temos, $\bar{X}^{c}={\overline{X^{c}}}^{c}$

iii. Se $X \subseteq Y \subseteq E$ então $\bar{X}^{c} \subseteq \bar{Y}^{c}$

Considere agora a extensão $C_{1}$ de $C^{\prime}$ da fig.3.a. A linha ac é um fechado de $C^{\prime}$ cujo fecho em $C_{1}$ contém o novo ponto $p$. É fácil ver que o fecho em $C_{1}$ de qualquer fechado de $C^{\prime}$ contendo ac também conterá $p$, bastando observar que a função fecho preserva inclusões. Por exemplo, $p \in \overline{a b c d}^{C_{2}}$. Esse fato pode ser generalizado da seguinte forma. Dada uma extensão pontual $N$ de $C$ pelo ponto $p$, o conjunto $\xi(N)$ dos fechados $X$ de $C$ tais que $p \in \bar{X}^{N}$ tem a seguinte propriedade:

(F1) Se $X \in \xi(N)$ e $Y$ um fechado de $C$ tal que $X \subseteq Y$ então $Y \in Z(N)$

Bm particular, nos exemplos das fig.3.a, 3.b, 3.c temos $f\left(C_{1}\right)=\{a c, a b c d\}$, $\xi\left(C_{2}\right)=\{a b, a b c d\}, F\left(C_{3}\right)=\{a c, b d, a b c d\}$.

0 conjunto $\xi(N)$ tem também uma ontra propriedade importante. Tentemos identifica-la dentro do próximo exemplo. Suponha que queremos uma extensão pontual $C_{4}$ de $C^{\prime}$ tal que $p \in \overline{a b}^{c_{d}}$ e $p \in \overline{c b}^{C^{4}}$. A propriedade (F1) garante que $p \in \overline{a b \cup c b}^{C}$. Denotemos o posto de $C^{\prime}$ por $\rho^{\prime}$ o posto de $C_{4}$ por $p_{4}$. Pela submodularidade de $\rho_{1}$ temos,

$$
\rho_{4}\left(\overline{a b}^{c_{6}}\right)+\rho_{4}\left(\overline{c b}^{c_{6}}\right) \geq \rho_{4}\left(\overline{a b}^{c_{4}} \cup \overline{c b}^{c_{4}}\right)+\rho_{4}\left(\overline{a b}^{c_{6}} \cap \overline{c b}^{c_{4}}\right)
$$

Por outro lado, os fechados $a b$ e cb de $C^{\prime}$ formam um par modular em $C^{\prime}$, isto é,

$$
\rho^{\prime}(a b)+\rho^{\prime}(c b)=\rho^{\prime}(a b \cup c b)+\rho^{\prime}(a b \cap c b)
$$

Mas, $_{1}\left(\overline{a b}^{c_{4}}\right)=p^{\prime}(a b), p_{4}\left(\overline{c b}^{c_{4}}\right)=p^{\prime}(c b)+p_{4}\left(\overline{a b}^{c_{0}} \cup \overline{c b}^{c_{c}}\right)=\rho^{\prime}(a b \cup c b)$, portanto (I) e $(I I)$ implicam que $p \in \bar{b}^{C_{6}}=\overline{a b \cap c b}^{C_{6}}$, ou de maneira equivalente, $p_{4}\left(\bar{b}_{p}{ }^{C_{6}}\right)=$ 1. Essa sitnação não tem representação numa configuração de pontos de um espaço projetivo. Apesar disso, damos na fig.4 uma representação alternativa para uma 


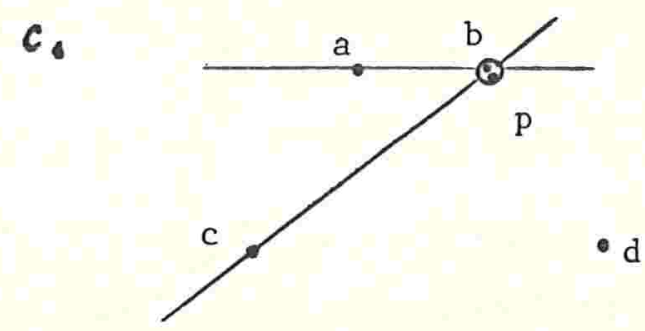

fig.4

possivel extensão $C_{4}$ onde os pontos $b$ e $p$ são colocados no interior de uma mesma circunferência, representando na realidade um único ponto. $F(N)$ :

Raciocínio análogo pode ser feito para concluir a segunda propriedade de

(F2) Se $X, Y \in \xi(N)$ formam um pas modular de $C$ então $X \cap Y \in \mathcal{F}(N)$

Um subconjunto do conjunto de fechados de um matroide que satifar (F1) ( (F2) chamado de filtro modslar. Em 1965 uma nova direça nos estudos sobre matróides foi dada por Crapo em seu trabalho sobre extensões pontuais $[\mathrm{Cr}]$. Nele, Crapo mostra que cada altro modular de um matrbide determina uma única extensão pontual. Para entender melhor a relação existente entre os filtros modulares e as extensões pontuais, é conveniente olharmos para os reticulados geométricos associados a matróide e às suas extensões pontuais. Examinamos Da fig.5 os reticulados dos fechados de $C^{\prime}$ e $C_{2}$ da fig.3.b.

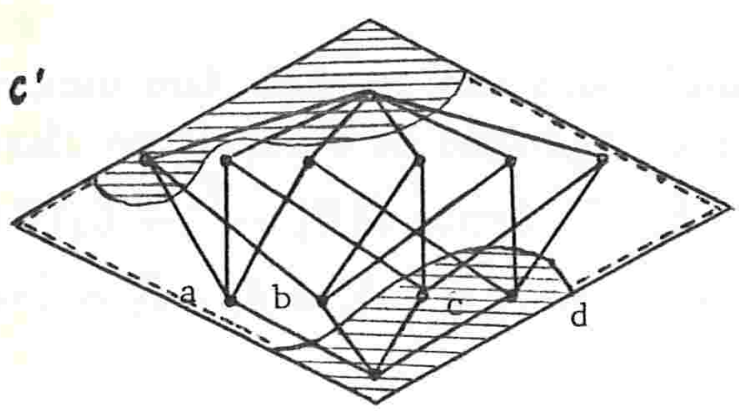

fig.8.6

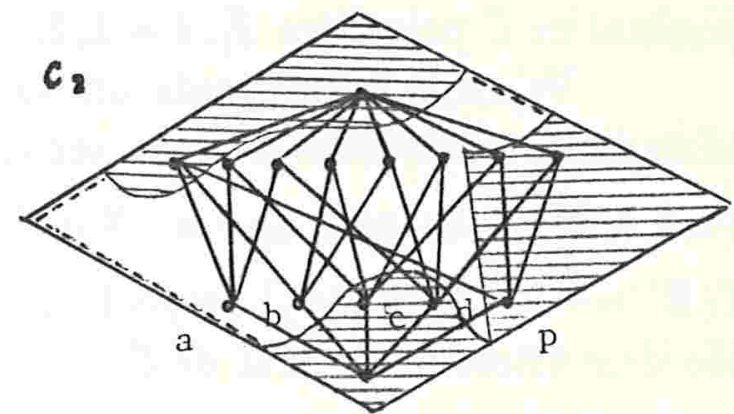

fig.b.b

$\mathrm{Na}$ fg.5.a, distingüimos 3 regiões. A regiz̃o hachurada superior representa o filtro modular $\xi\left(C_{2}\right)$ e a região não hachurada contém os fechados de $C^{\prime}$ cobertos por algum elemento de $\xi\left(C_{2}\right)$. Na fig.5.b, a regiäo hachurada contém os fechados de $\xi\left(C_{3}\right)$ acrescidos do ponto $D$ com posto inalterado. $O_{B}$ fechados de $C^{\prime}$ fora do tiltro continuam sendo fech -dos de $C_{2}$ e também não têm alteração de posto. Finalmente, quando acrescentamos $p$ a um fechado de $C^{\prime}$ não coberto por elemento do filtro obtemos um fechado de $C_{3}$ com posto uma unidade major. $\mathrm{Na}$ realidade, - que ocorre nesse exemplo também ocorre no caso geral; a partir de um filtro modular $\xi$ de um matróide $M$ sobre um conjunto finito $E$ dado por seu reticulado 


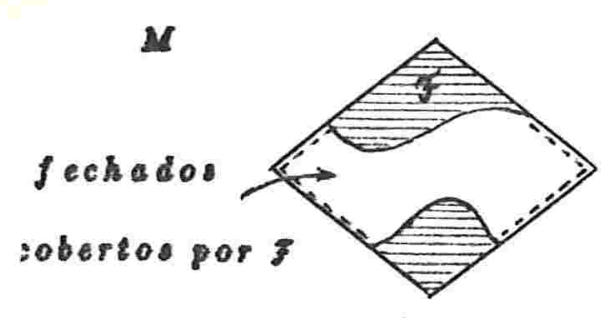

fig.6.

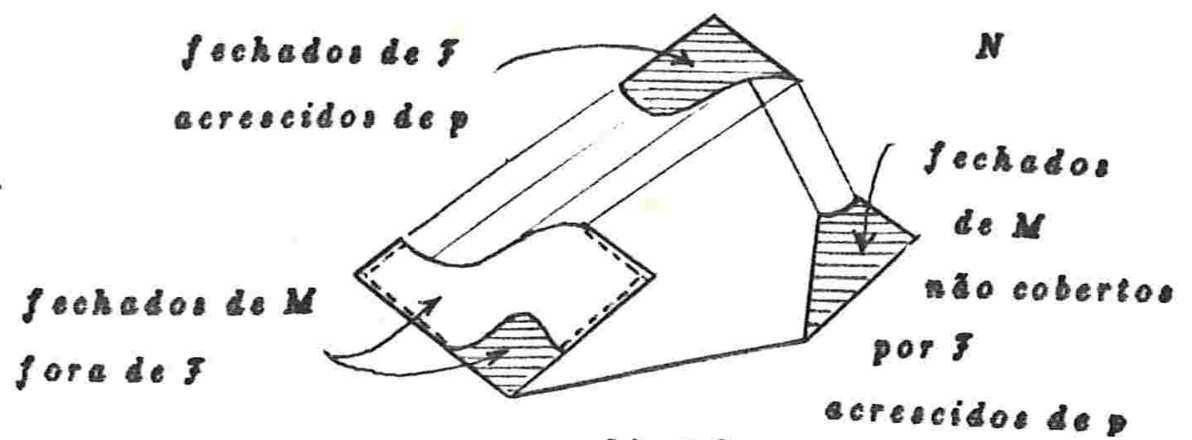

fig.6.6

de fechados, podemos obter o reticulado de fechados de uma extensão pontual $N$ tal que $\bar{\xi}=\bar{\xi}(N)$ da forma descrita na fig. 6 .

Usaremos a notação, $M(E) \stackrel{\xi}{\longrightarrow} N(E \cup p)$ para dizer que $N$ é a extensão pontual de $M$ sobre $E$ U p determinada pelo fltro modular $\xi$ de $M$.

Vale observar que com essa construção, a extensão pontual obtida a partir de um filtro modular de nma configuração de pontos, apesar de ser um matróide, pode não ser representável como uma configuração de pontos num espaço projetivo. A extensão $M_{4}$ da fig.4 é um exemplo dessa situação. Várias classes de matroides apresentam essa "anomalia", isto $\varepsilon$, não são fechadas por extensões pontuais.

Analisemos agora o seguinte problema: dados dois filtros modulares $I_{1}$ e $I_{2}$ da configuração $C$ perguntamos se é possível colocar um novo ponto $p_{1}$ exatamente nos fechados de $\gamma_{1}$ e ao mesmo tempo um novo ponto $p_{2}$, distinto de $p_{1}$, exatamente nos fechados de $\bar{\gamma}_{2}$. Uma outra formulaçã para essa pergunta é se existe uma extensão $C^{\prime}$ de $C$ sobre $E \cup p_{1} \cup p_{2}$ tal que $C^{\prime}\left(E \cup p_{i}\right)$ é exatamente a extensão pontual de $C$ pelo iltro $s_{i} i=1,2$.

Vejamos inicialmente um exemplo onde essa pergunta tem uma resposta afirmativa. Considere $\mathcal{C}$ como sendo a configuração de 4 pontos no plano, $E=$ $\{a, b, c, d\}$, dada pela fig.7.a. Nas fig.7.b e 7.c temos $C(E) \stackrel{z_{1}}{\longrightarrow} C_{1}\left(\bar{E} \cup p_{1}\right)$ e $C(\bar{E}) \stackrel{F_{2}}{\longrightarrow} C_{2}\left(E \cup p_{3}\right)$ respectivamente, onde $F_{1}=\{a b, a b c d\}$ e $F_{2}=\{a d, a b c d\}$ são dois filtros modulares de $C$.

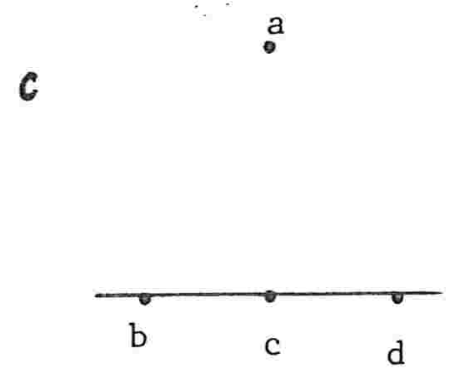

fig.7.6

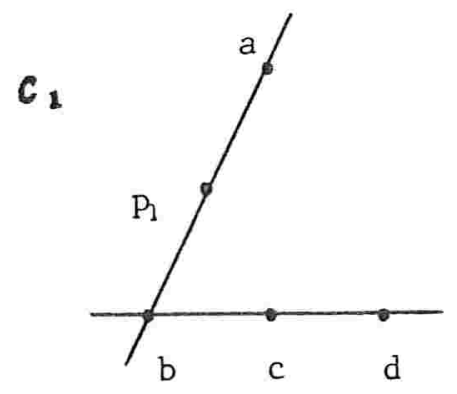

fig.7.6

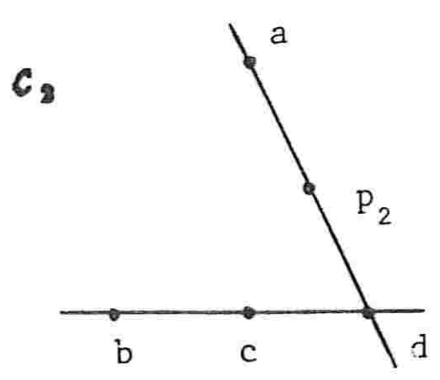

fig.7.6

Duas possiveis extensões pontuais comuns a $C_{1}$ e $C_{3}$ são dadas pelas fig.8.a e 8.b. 


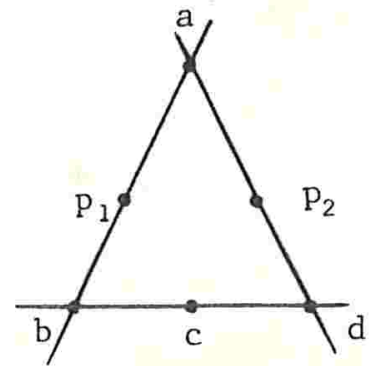

fig.8.

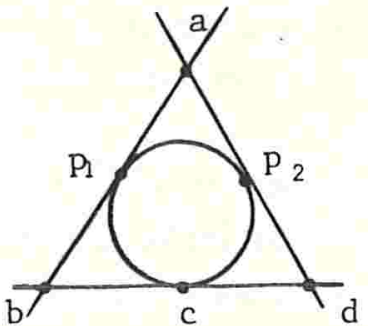

fig.8.8

Considere agora $C$ como sendo a configuração de 6 pontos no plano, $E=$ $\{a, b, c, d, e, f\}$, na qual näo existem 3 pontos colineares e $\xi_{1}=\{a b, c d, E\}, \xi_{2}=$ $\{a b, c d, e f, E\}$ dois 6litros modulares. As fig.9.a e 9.b mostram as respectivas extensōes pontuais $C_{1}, C_{2}$ por esses dois filtros.

$c_{1}$

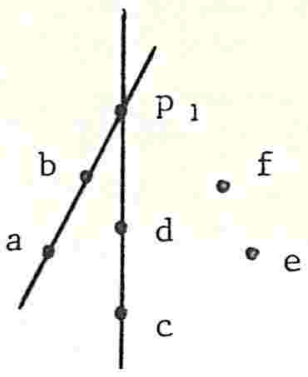

fis.9.9.

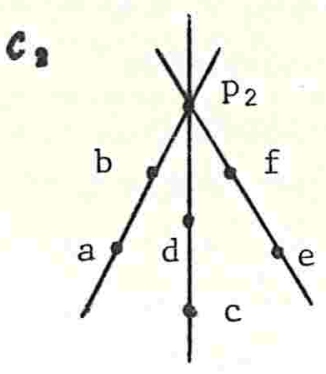

fig.9.6

Se em $C_{1}$ tentamos colocar o ponto $p_{2}$ nos fechados de $\xi_{2}, p_{1}$ é forçado a pertencer à linha $e f$, uma contradição. Por outro lado, em $C_{3}$ não é possivel colocar $p_{1}$ somente nos fechados de $\mathcal{F}_{1}$. Assim, $C_{1}$ e $C_{2}$ são extensōes incompativeis de $C$.

0 principal objetivo dessa dissertação ef estudar uma versão geral desse allimo problema: dados um matróide $M$ e as extensóes $N_{1}, N_{3}, \ldots, N_{n}$ de $M$, será que existe uma extensão comum a todas elas? Em caso afirmativo diremos que $N_{1}, N_{2}, \ldots, N_{n}$ são eatensōes compat́veis de $M$. Essa pergunta permanece até boje sem uma boa resposta, isto é, ఐão existe uma caracterisação completa para a com. patibilidade de extensões. Mesmo quando restringimos o problema ds extensões pontuais de $M$ esse quadro permanece praticamente inalterado. A maioria dos trabalhos sobre compatibilidade de extensões está restrita a essa versão simplificada do problema. Cordovil em [Co2] apresentou uma condição suficjente interessante para que as extensões pontuais $N_{1}, N_{3}, \ldots, N_{n}$ de um matróide sejam compatíveis, fornecendo uma caracterisação de uma extensão especial $N$ comum a $N_{1}, N_{2}, \ldots, N_{n}$. No caso de pares de extensóes poutuais de um matróide, o problema da compatibilidade está completamente resolvido. Vários autores desenvolveram diferentes teorias e técnicas para obter uma solução para esse problema particular:

- Las Vergnas em [LV1] trabalhou com relações de inclusão entre os fechados dos fltros modulare associados ao par de extensōes pontuais. 
- Cordovil em [Co2] uson uma generalização do conceito de filtro modular introdugido por Las Vergnas em [LV?].

- Cheung em $[\mathrm{Ch}]$ obteve 3 soluções distintas baseadas, respectivamente, na naça de fecho de um filtro modvlar, nas famflias dos hiperplanos de filros modulares e nas operaçōes de restrição e contração de matróides.

A seguir, prefendemos transmitir de maneira informal as principais idéiss mencionadas no último parágrafo, ainda dentro da classe dos matróides aobre configuraçōes de pontos de um espaço projetivo.

Diremos que os filtros modulares de um matroide são compatíveis se e 80mente se as extensões pontuais do matróide determinadas por esses filtros também - forem. Assim, ao invés de tratarmos do problema da compatibilidade de extensoes pontuais, vamos tratar do problema da compatibilidade de filtros modulares, que é equivalente.

Observando com mais cuidado os exemplos das fig.7.a, 7.b e 7.c, notamos que quando estendemos a configuração $C$ pelo filtro $\Psi_{1}$ obtendo a configuração $C_{1}$, os fechos dos elementos de $f_{3}$ em $C_{1}$ formam um filtro modular de $C_{1}$. Podemos então estender $C_{1}$ por esse filtro obtendo a extengão da fig.8.a, comum a $C_{1} e$ $C_{2}$. O mesmo raciocínio é aplicável trocando $\xi_{1}, C_{1}$ por $F_{3}, C_{3}$, reapectivamente. 0 interessante nesse último caso, é que continuamos a obter a mesma extensão pontual da îg.8.a. Isto não é coincidềncia... No entanto, no exemplo da f́g.9, 20 estendermos $C$ pelo filtro modular $\xi_{1}$ obtendo $C_{1}$, os fechos em $C_{1}$ dos elementos de $F_{2}$ a ão formam um filsro modular pois $\overline{a b}^{C_{2}}, \overline{c d}^{C_{2}}$ formam um par modular de $C_{1}$ e a intersecção $\overline{a b}^{C_{2}} \cap \overline{c d}^{C_{1}}=p_{1}$ não é fecho em $C_{1}$ de nenhum elemento de $\mathcal{F}_{2}$.

Mas o que está por trás dessea fatos? 真 rasodvel perguntar, messes dois exemplos, se essa "preservação" da estrutura de $\xi_{2}$ na extensão pontual $C_{1}$ de $C$ pelo flltro $F_{1}$ tem alguma relaçäo com a compatibilidade dos filtros $F_{1}, F_{2}$ que, como já observado antes, é verdadeira no exemplo da fig.7 e falsa no exemplo da fig.9. Quando falamos em "preservaç̃o" de $\xi_{2}$ em $C_{1}$ estamos querendo diser que os elementos minimais de $F_{3}$ em $C$ quando fechados em $C_{1}$ são exatamente os elementos minimais de um filtro modular de $C_{1}$. Isto porque, visto a condição (FI), o flitro é determinado pelos seus membros minimais. Se isso ocorrer, diremos que $\xi_{1}, \xi_{3}$ são filtros modulares ultra-compativeis. Perceba que os filtros $\xi_{1}, \xi_{2}$ do exemplo da fig. 9 não são ultra-compatíveis porque $a b, c d \in \xi_{1} \cap \xi_{2}, \emptyset=$ abกcd $\notin \xi_{1} \cup$ $\delta_{2}$ e $\rho_{c}(a b)+\rho_{c}(c d)-\rho_{c}(a b \cup c d)-\rho c(a b \cap c d)=10$ que fag com que o par $\overline{a b} c_{2}, \frac{c d}{c} c_{2}$ torne-se modular em $C_{1}$, forçando $p_{1}=\overline{a b}^{c_{1}} \cap \overline{c d}^{C_{1}}$ a pertencer a todo filtro modular que contenha $\overline{a b}^{C_{1}}$ e $\overline{c d}^{C_{1}}$. Esse fato caracteriga a ultra-compatibilidade de um par $\xi_{3}, \xi_{2}$ de filtros modulares de um matróide $M: \xi_{1}, \xi_{2}$ são ultra-compatíveis se e somente se não existem $X, Y \in \xi_{1} \cap \xi_{2}$ tais que $X \cap Y \notin f_{1} \cup \xi_{2}$ e de $f_{M}(X, Y)=1$, 
onde de $f_{M}(X, Y)=\rho_{M}(X)+\rho_{M}(Y)-\rho_{M}(X \cup Y)-\rho_{M}(X \cap Y)$ chamado defeito do par de fechados $X, Y$ em $M$. Informalmente, podemos pensar no defeito como uma medida do quão "longe" um par de fechados está de ser modular. Esasa definição de ultra-compatibilidade mostra que a ordem dos filtros $\xi_{1}, \xi_{2}$ que parecia importante na def̂nição inicial, é irrelevante. Aliss, a obtenção da mesma extensão da fig.8.a por dois caminhos distintos discutidos acima é consequência dessa observação.

O Capitulo I desse trabalho é dedicado principalmente ao estudo da ultracompatibilidade no caso geral, onde todas as idéias apresentadas acima são generalisadas para uma sequência $f_{1}, \tau_{2}, \ldots, \tau_{n}$ de filtros modulares de um matróide $M$ sobre $E$.

Dado um conjunto inito $P$ arbitrário disjunto de $E$, seria muito bom se congeguíssemos uma bijeção entre as extensões $N$ de $M$ sobre $E \cup P$ e alguma estrutura de fechados de $M$, a exemplo do que ocorre com os filtros modulares e as extensōes pontuais de $M$. Uma primeira extensão da idéia de filtro modular, proposta por Las Vergnas em [LV1], \& rasoavelmente simples mas o preço pago é a perda da bijeção. Tomemos por exemplo a configuração $C$ sobre 4 pontos no espaço, $E=\{a, b, c, d\}$, na qual não existem 3 pontos colineares e cada um dos pontos não pertence ao plano determinado pelos 3 outros. Coloque um novo ponto $p_{1}$ na linha ab um novo ponto $p_{2}$ na linha bc obtendo a extensão $C_{1}$ de $C$ da fig. 10.b.

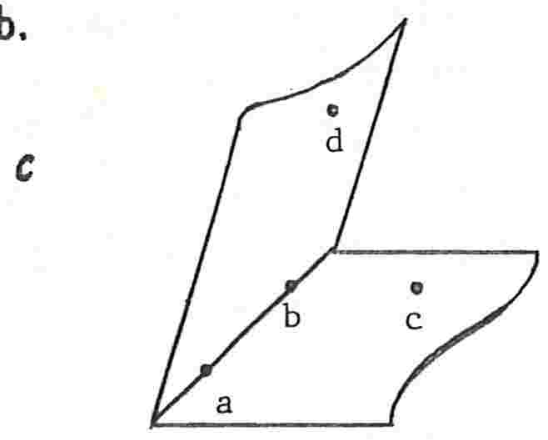

fig.10.e

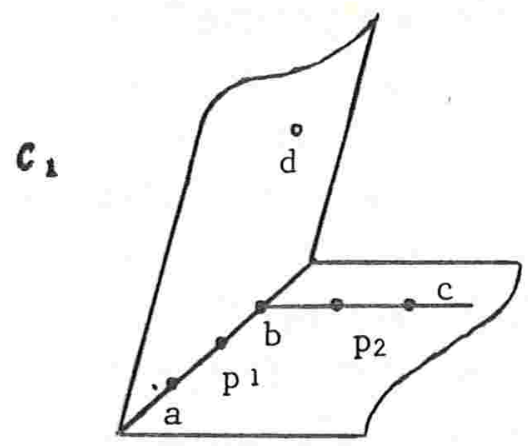

fig.10.b

Os conjuntos $\mathcal{E}^{(i)}=\left\{X\right.$ fechado de $\left.C: P C_{1}\left(X \cup p_{1} \cup p_{2}\right)-p c(X)=i\right\}$, $i=0,1,2$, formam uma partição dos fechados de $C$ chamado empilhamento da ezstensõo $C_{1}$ de $C$ pelo acréscimo da linha $p_{1} p_{3}$. As seguintes propriedades são verificadas:

(E1) Se $X, Y$ são fechados de $\mathcal{C}, Y \in \mathcal{C}^{(i)}$ e $X$ cobre $Y$ (isto $\hat{e}, Y \subseteq X$ e não existe $Z$ fechado de $C$ tal que $Y \subset Z \subset X)$ então $X \in \mathcal{E}^{(i)} \cup \mathcal{E}^{(\bar{i}-1)}$

(E2) Se $X, Y$ são fechados de $C, X, Y$ cobrem $X \cap Y$ $X, Y, \overline{X \cup Y}^{c} \in \mathcal{E}^{(i)}$ entäo $X \cap Y \in \mathbb{R}^{(i)}$

Olhando para o reticulado dos fechados de $C$ temos eqquematicamente: 


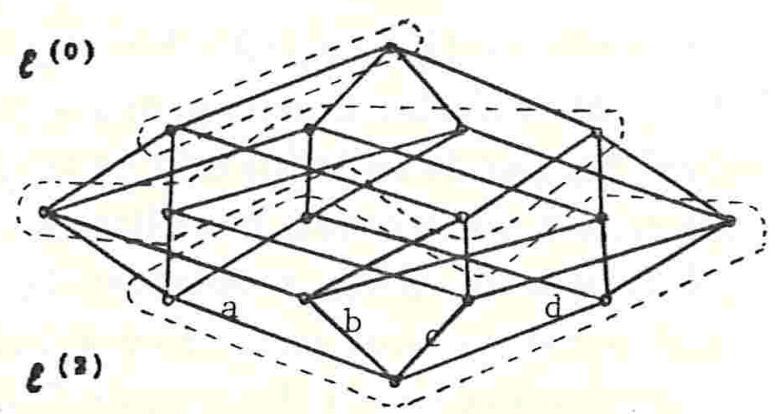

fig.11

Em particular as propriedades (E1) e (E2) mostram que $\varepsilon^{(0)}$ é um filtro modular. Infelizmente, existem várias extensões de $C$ obtidas pelo acréscimo da linha $p_{1} p_{2}$ com esse mesmo empilhamento (veja ig.12.a, 12.b e 12.c).

C.

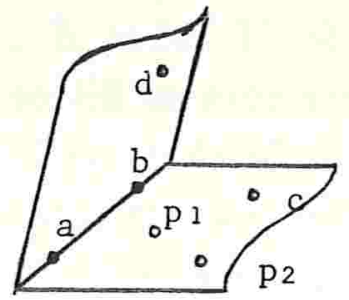

fig.12.6
$C_{8}$

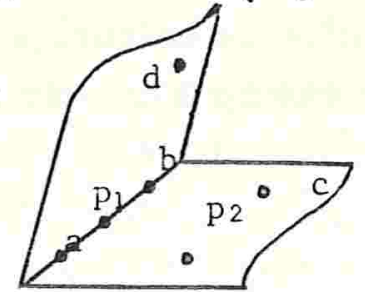

$f$ ig.12.6

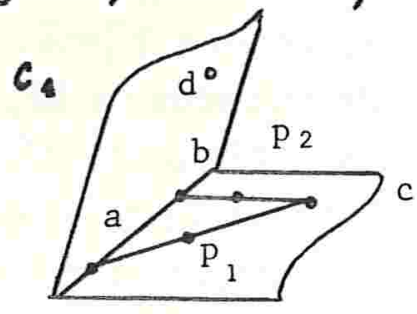

fig.12.e

Entretanto, conseguimos dentre todas essas extensōes, uma extensão especial "o mais livre possivel", isto é, uma extensão obtida de $C$ colocando-se a linha $p_{1} p_{2}$ interferindo o menos possivel com os fechados de $C_{1}$ tomando o cuidado de manter o empilhamento. Essa extensão é exatamente $C_{2}$ da fig.11.a. Note que essa idéa de "o mais livre posstvel" é equivalente a: se $C^{\prime}$ é uma extensão de $C$ pelo acréscimo de $p_{1} p_{2}$ com mesmo empilhamento de $C_{3}$ então $p C_{8}(X) \geq p C^{\prime}(X)$ para todo $X \subseteq E \cup p_{1} \cup p_{3}$. Chamaremos $C_{3}$ de estensão normal de $C$ determinada pelo empilhamento $\mathcal{E}^{(0)}, \mathcal{E}^{(1)}, \mathcal{E}^{(2)}$ e pela linha $p_{1} p_{3}$.

As extenso normais e os empilbamentos serão ferramentas importantes nas caracterizaçōes de pares de filtros modulares compatíveis, devidas a Las Vergnas e Cordovil apresentadas no Capítulo II.

Como já dissemos, o objetivo dessa introdução trangmitir ao leitor as principais idéias envolvidas neste trabalho, de una maneira o mais natural possivel, sem entrar em déalhes técnicos desnecessários e não pressupondo nenhum conhecimento profundo da Teoria dos Matróides. Acreditamos nesse ponto que esse objetivo já foi atingido. Vamos nos permitir então ser um pouco mais breves nas descrições dos dois últimos capítulos da disserbação.

Nos Caṕtulos III IV abordamos indiretamente o problema da compatibilidade de filtros modulares através de duas estruturas que estão em correspondência bijetora com os filtros modulares.

A primeira delas ja 6 bastante conhecida e foi introdusida por Crapo em [Cr]. Assim como um filtro modular completamente determinado por seus ele- 
mentos minimais, os hiperplanos presentes no filtro, isto é, os fechados do filtro com posto uma unidade menor que o posto do matróide, também dão uma descrição completa do filtro. Essa famflia de hiperplanos do filtro é chamada subclasse linear. Dessa forma, a compatibilidade de filtros modulares indus naturalmente a noção de compatibilidade de subclasses lineares. No Capitulo III, detetamos exatamente a situação que bloqueia a compatibilidade de duas subclasses lineares comparáveis por inclusão.

Passemos a descrever a segunda estrutura. Dado um matroide $M$ sobre um conjunto $E$, trabalharemos no Capitulo IV com matróides sobre o mesmo $E$ cujas famllias de fechados são subfamilias da família dos fechados de $M$. Tais matróides são denominados quocientes de $M$. E possivel caracterigar uma extensão qualquer de $M$ a partir de feizes de quocientes de $M$ que nada mais são que famflias de quocientes de $M$ com determinadas propriedades. Não vamos aqui listar essas propriedades mas podemos adiantar que elas, bem como toda teoria de quocientes, estão relacionadas com uma operação muito importante sobre matróides, a contraçäo. A contração de matróides pode ser vista como uma abstração da operação de contração de arestas num grafo. Do ponto de vista de configurações de pontos, uma contração corresponde à projeção da configuração na direção de um de seus fechados. Se a diferença entre o posto do matróide $M$ e o posto de um quociente é 1,0 quociente é dito elementar. No Capítulo IV estabelecemos uma bijeção entre os quocientes elementares e os filtros modulares de um matróide, tratando em seguida do problema da representabilidade de quocientes elementares que quando ollhado em termos dos filtros modulares associados corresponde ao problema da compatibilidade de filtros. 



\section{Indice}

Agradecimentos

Abstract

vii

Introdução

xi

Preliminares

1. Notação Básica

2. Matróides

3. Extensöes

4. Morfismos Fortes 10

Capítulo I: Ultra-Compatibilidade 13

1. A Ultra-Compatibilidade implicando Compatibilidade 14

2. Extensöes Lisas 22 
Capítulo II: Extensões Normais e Empilhamentos 31

1. Normalidade 32

2. Empilhamentos 34

3. O Semi-reticulado das 2-Extensões 46

4. 2-Compatibilidade 55

Capitulo III: Compatibilidade de Subclasses Lineares 69

1. A Relação entre Subclasses Lineares e Filtros Modulares 70

2. Compatibilidade de Pares de Subclasses Lineares 72

Capitulo IV: Representabilidade de Quocientes 69

1. A Relação entre Quocientes e Filtros Modulares 70

2. Feixes de Quocientes 72

3. Sequências de Rebaixamentos e Levantamentos 87

4. Representaçóes de Quocientes 93

Referências Bibliográficas $\quad 99$

$\begin{array}{ll}\text { fndice Remissivo } & 101\end{array}$ 


\section{Preliminares}

O propósito dessas Preliminares é familiariaar o leitor com conceitos básicos e alguns resultados da Teoria dos Matróides a serem utilizados no restante do texto. Tentamos, na medida do possível, nos ater à terminologia utilizada por Welsh em [We]. Grande parte das demonstraçōes dos resultados aqui apresentados podem ser encontradas em [We] e [Ai].

Inevitavelmente, essas Preliminares contêm um número muito grande de definições e resultados que provavelmente não serão absorvidos numa primeira leitura. Aconselhamos o leitor a passar rapidamente por eles e retornar sempre que necessário.

$N_{2}$ Seção 2, após uma breve revisão da notação básica feita na Seção 1 , apresentamos várias definições axiomáticas equivalentes de matrróides, bem como algumas operaçōes sobre eles. Na Seção 3 , introdusimos o conceito de extensões de matróides, objeto principal de investigação deste texto. A Seção 4 é dedicada a um tipo especial de função entre matróides, o morfismo forte, que será muito útil numa construção feita no Capfłulo IV.

Assumimos alguma familiaridade com conceitos básicos da Teoria de Conjuntos, Ordens, Álgebra Linear, Reticulados, Teoria dos Grafos e Geometria Projetiva. 


\section{NOTACAOO BASICA}

Adotaremos as convenções nsuais da teoria de conjuntos. Os símbolos e $\Delta$ serão usados, respectivamente para a diferença e diferença simétrica entre conjuntos.

Os conjuntos (resp., elementos) serão denotados por letras maíusculas (resp., minúsculas) em it́lílco. Usualmente $\left\{x_{1}, x_{2}, \ldots, x_{n}\right\}$ denota o conjunto com elementos $x_{1}, x_{3}, \ldots, x_{n}$ mas quando estiver claro no contexto que estamos nos referindo ao conjunto e não aos elementos, abreviaremoso conjunto $\left\{x_{1}, x_{3}, \ldots, x_{n}\right\}$ por $x_{1} x_{2} \ldots x_{n}$. Por exemplo, $X \cup x$ denota $X \cup\{x\}$.

\section{MATRÓIDES}

Um matroide pode ser definido de várias maneiras diferentes porém equivalentes. Comecemos com a definição que nos parece mais natural.

Um motróide $M$ é um par $(E, I)$ onde $E$ é um conjunto finito e $I$ é uma famflia de subconjuntos de $E$, denominados independentes, tal que para todos $X$, $Y \subseteq E$

(II) $\emptyset \in I$

(I2) $X \subseteq Y$ $X \in I \Rightarrow X \in I$

(I3) Se $X, Y \in I$ e $|X|>|Y|$ então existe $x \in X-Y$ bal que $Y \cup x \in I$

Ao invés de mencionarmos o par $(E, /)$ vamos apenas referir-nos a matróide $M$ sobre $E$, ou ainda, $M(E)$.

Exemplos de Matroides:

1. Matrobides Lineares

$E \subseteq V$ espaço vetorial sobre um corpo ou anel de divisâo $F$

$I=\{I \subseteq E: I$ é linearmente independente em $V\}$

2. Matroides Uniformes

$B$ conjunto de elementos

$I=\{I \subseteq E:|I| \leq k\} \quad 0 \leq k \leq n, k$ fixo $\mathcal{L}_{k}(\bar{E})=(E, \check{I})$

Obs:: Se $E=n$ então $\mathcal{L}_{k}(E)$ é chamado matróide liere de posto $n$, denotado apenas por $\mathcal{L}(E)$ 
3. Matroides Gróficos

$E=E G$ conjunto das arestas de um grafo $G$

$I=\{I \subseteq E: I$ não contém circuitos $\}$

$M(G)=(\mathbb{E}, \zeta)$

4. Matroides Thanstersais

$E=V_{1} G$ conjunto dos vértices de uma classe de bipartição de um grafo bipartido $G$

$I=\{I \subseteq E: I$ é emparelhável $\}$

5. Matroides Afins

$B \subseteq V$ expaco vetorial sobre um corpo $F$

$I=\{I \subseteq E: I$ é independente afim $\}$

Obs.: $\left\{v_{1}, v_{2}, \ldots, v_{k}\right\}$ é independente afim se não existem $\lambda_{1}, \lambda_{2}, \ldots, \lambda_{k} \in F$ não todos nulos tais que

$$
\sum_{i=1}^{k} \lambda_{i} v_{i}=0 \quad \text { e } \quad \sum_{i=1}^{k} \lambda_{i}=0
$$

Por todo o restante do texto, $M$ será um matróide sobre o conjunto finito $E$.

Por analogia com espaços vetoriais, fazemos as seguintes definições:

(a) $X \subseteq E$ é base de $M$, se $X$ é independente maximal

(b) O posto de $X \subseteq E$ em $M$, denotado por $\rho_{M}(X)$, é a cardinalidade de uma base de $X .0$ posto do matróide $M$, denotado por $\rho M$, c o natural $\rho_{M}(E)$.

(c) 0 fecho de $X \subseteq E$ em $M$, denotado por $\bar{X}^{M}$, é o conjunto $\{z \in E$ : $\left.\rho_{M}(X \cup x)=\rho_{M}(X)\right\}$

(d) $X \subseteq E$ é dependerte em $M$, se $X$ não é independente

(e) $X \subseteq E$ é circurito de $M$, se $X$ é dependente minimal. Os circuitos de cardinalidade 1 (resp., 2) são chamados de laços (resp., elementos paralelos)

(1) $X \subseteq E$ é jechado de $M$, se $\bar{X}^{M}=X$. Os fechados de posto $1,2,3, \rho M-1$, $p M-2$ e $p M-3$ são chamados, respectivamente, de pontos, birtur, plenon, copontos (ou hiperplanos), colinhas e coplanos.

O conhecimento das bases, ou posto, ou fecho, on circuitos ou hiperplanos é suficiente para determinar o matróide de modo único: 
Axlomas de Bases Uma famflia $B$ de subconjuntos de $E$ é a famflia das bases de um matróide sobre $E$ se e somente se

(B1) $B \neq 0$

(B2) Existe $n$ tal que $|B|=n$ para todo $B \in B$

(B3) $P$ ara bodos $B_{1}, B_{2} \in B$ e $x_{1} \in B_{1}-B_{2}$ exisie $x_{2} \in B_{2}-B_{1}$ tal que $\left(B_{1} \cup \Sigma_{2}\right)-\Sigma_{1} \in B$

Axiomas de Posto Uma função $\rho$ do conjunto das partes de $\boldsymbol{E}$ no conjunto dos inteiros é a função posto de um matróide sobre $E$ se e romente se para todos $X \subseteq E, y, z \in E$

(RI) $\rho(0)=0$

(R2) $\rho(X) \leq \rho(X \cup y) \leq \rho(X)+1$

(R3) $\operatorname{Se} \rho(X \cup y)=\rho(X \cup z)=\rho(X)$ eกติ๊o $\rho(X \cup y \cup z)=\rho(X)$

Os axiomas de posto acima são os efetivamente nsados no decorrer do texto. Entretanto, algumas propriedades da função posto ficam mais claras quando olhamos para o seguinte sistema de axiomas equivalentes a (R1), (R2) e (R3):

para todos $X, Y \subseteq E$,

$\left(R 1^{\prime}\right) 0 \leq \rho(X) \leq|X|$

$\left(\mathrm{R} 3^{\prime}\right) X \subseteq Y \Rightarrow \rho(X) \leq \rho(Y)$

(R3) $\rho(X)+\rho(Y) \geq \rho(X \cup Y)+\rho(X \cap Y)$

Axiomas de Fecho Uma função ${ }^{-}$do conjunto das partes de $E$ no conjunto das partes de $E$ é a função fecho de um matróide sobre $E$ se e somente se para todos $X, Y \subseteq E$ e $x, y \in E$

(Fel) $X \subseteq \bar{X}$

(Fe2) $Y \subseteq X \Rightarrow \bar{Y} \subseteq \bar{X}$

(Fe3) $\bar{X}=\overline{\bar{X}}$ 
(Fe4) Se $y \notin \bar{X}$ e $y \in \overline{X \cup x}$ então $x \in \overline{X \cup y}$

Axlomas de Circuitos Uma famflia $C$ de aubconjuntos de $E$ é a família dos circuitos de um matróide sobre $E$ se e somente se

(C1) $₫ C$

(C2) $C_{1}, C_{2} \in C$ e $C_{1} \subseteq C_{2} \Rightarrow C_{1}=C_{2}$

(C3) Se $C_{1}, C_{2} \in C$ distintos e $x \in C_{1} \cap C_{2}$ então existe $C_{3} \in C$ tal que $C_{3} \subseteq$ $\left(C_{1} \cup C_{3}\right)-x$

Axiomas de Hiperplenos Uma famflia $K$ de subconjuntos de $E$ é a famflia dos hiperplanos de um matróide sobre $E$ se e somente se para lodos $\mathbb{H}_{1}, H_{2} \in$ X distinsos

(H1) $E \notin$

(H2) $H_{1} \& H_{2}$

(H3) Se $x \in E-\left(H_{1} \cup H_{2}\right)$ então existe $H \in X$ tal que $\left(H_{1} \cap H_{2}\right) \cup x \subseteq H$

A familia dos independentes, das bases, dos circuitos, dos fechados e dos hiperplanos do matróide $M$ serão denotadas por $I_{M}, B_{M}, C_{M}, \bar{F}_{M}$ e $X_{M}$, respectivamente.

Podemos associar ao matróide $M$ o conjunto $\mathcal{L}(M)$ ordenado por inclusão cujos elementos são os fechados de $M$. Na realidade, $\mathcal{L}(M)$ é um reticulado onde para todos $X, Y \in \mathcal{I}_{M}$,

$$
0=\bar{\emptyset}^{M} \quad 1=E \quad X \wedge Y=X \cap Y \quad X \vee Y=\overline{X \cup Y}^{M}
$$

$\mathcal{L}(M)$ será chamado de reticulado do matróide $M$. Lembremos alguns conceitos sobre reticulados. Um reticulado $P$ é graduado se admite uma funçäo altwra, isto $e ́$, uma função $h$ de $P$ nos inteiros que satisfaz, para todos $z, y \in P$,

$$
\text { i. } x<y \Rightarrow h(x)<h(y)
$$


ii. y cobre $x$, igto é, $x<y$ e não existe $z \in P$ tal que $x<z<y \Rightarrow h(y)=$ $h(x)+1$

Um reticulado graduado é dito semimodelar se para todos $x, y \in P$,

$$
h(x)+h(y) \geq h(x \vee y)+h(x \wedge y)
$$

Note que $\mathcal{C}(M)$ é semimodular com função altura $p_{M}$. E natural perguntar se existe o caminho inverso, ou melhor, se a partir de um reticulado é possivel obter um matróide. Para responder essa pergunta uma definição se fan necessária. Um reticulado geométrico se ele for semimodular, siver altura finita e se todo elemento

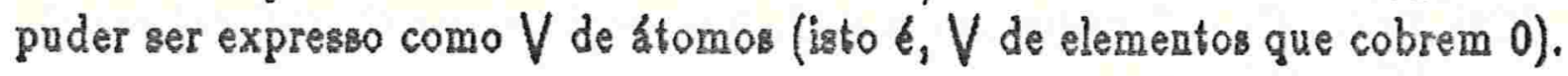

Teorema (Reticulados Geométricos) Um reticulado fnito fo geométrico se e somente se ele for jomorfo a reticulado de um matroide.

A seguir apresentaremos três operaçốes sobre matroides muito úteis nas provas por indução. Todas elas têm por objetivo obter matróides "menores" que preservam de algum modo a estrutura do matróide original.

Teorema (Truncamento) Seja $1 \leq k \leq \rho M$. Então

$$
I_{M_{k}}=\left\{X \in I_{M}:|X| \leq k\right\}
$$

é a famflia dos independentes de um matróide sobre $E$, denotado por $M_{k}$, truncamento de $M$ ao nível k. Nesse caso,

$$
\begin{aligned}
& C_{M_{k}}=\left\{C \in C_{M}:|C| \leq k+1\right\} \cup\left\{I \in I_{M}:|I|=k+1\right\} \\
& B_{M_{k}}=\left\{I \in I_{M}:|I|=k\right\} \\
& {\rho \mathbb{N}_{k}}_{k}(X)=\min \left(\rho_{M}(X), k\right), \quad X \subseteq E \\
& \bar{X}^{M_{k}}= \begin{cases}\bar{X}^{M}, & \text { se } \rho_{M}(X)<k \\
\bar{E}, & \text { caso contrário, } \quad X \subseteq E\end{cases} \\
& \mathcal{F}_{M_{h}}=\left\{X \in \xi_{M}: \rho_{M}(X)<k\right\} \cup\{E\}
\end{aligned}
$$


Teorema (Re⿶trição) Seja $A \subseteq E$. Então

$$
I_{M \backslash A}=\left\{X-A: X \in I_{M}\right\}=\left\{X \in I_{M}: X \cap A=\emptyset\right\}
$$

é a famflia dos independentes de um matróide sobre $E-A$, denotado por $M \backslash A$ ou $M(E-A)$, restrição de $M$ a $E \cdot A$. Nesse caso,

$$
C_{M \backslash A}=\left\{C \in C_{M}: C \cap A=\emptyset\right\}
$$

$B_{M \backslash A}=\{B$ base de $E-A$ em $M\}=\left\{B-A: B \in B_{M},|B \cap A|\right.$ minimo $\}$

$$
\begin{array}{ll}
\rho_{M \backslash A}(X)=\rho_{M}(X), & X \subseteq E-\Lambda \\
\bar{X}^{M}=\bar{X}^{M}-\Lambda, & X \subseteq E-\Lambda
\end{array}
$$

$\mathcal{L}(M \backslash A)$ é isomorfo ao subreticulado $\left[0, \overline{E-A^{M}}\right]$ de $\mathcal{L}(M)$.

Teorem (Contração) Seja $A \subseteq E$. Então

$$
I_{M / A}=\left\{X \subseteq E-A: p_{M}(A \cup X)=\rho_{M}(A)+|X|\right\}
$$

é a familia dos independentes de um matróide sobre $E-A$, denotado por $M / A$, contração de M por A. Nesse caso,

$$
C_{M / A}=\operatorname{minimais} \operatorname{de}\left\{C-\Lambda: C \in C_{M}, C \nsubseteq \Lambda\right\}
$$

$B_{M / A}=\{B-A: B \cap A$ base de $A$ em $M\}=\left\{B-A: B \in B_{M},|B \cap A|\right.$ máximo $\}$

$$
\begin{gathered}
\rho_{M / A}(X)=\rho_{M}(\Lambda \cup X)-\rho_{M}(A), \quad X \subseteq E-A \\
\bar{X}^{M / A}=\overline{X \cup A}^{M}-\Lambda, \quad X \subseteq E-\Lambda
\end{gathered}
$$

$\mathcal{L}(M / A)$ é isomorfo ao subreticulado $\left[\bar{\Lambda}^{M}, 1\right]$ de $\mathcal{L}(M)$.

Podemos associar uma certa ordem parcial sobre a familia de todos os matróides sobre um conjunto finito fixo. Denote por $M(E)$ a famflia de todos os matróides sobre $E$. Sejam $M_{1}, M_{2} \in M(E)$. Dizemos que $M_{1}$ é masis livre (ou mais fraco) que $M_{3}$ se qualquer um dos itens equivalentes abaixo for satisfeito: 
j. Todo independente de $M_{2}$ é independente em $M_{1}$

ii. $\rho_{M_{2}}(X) \geq \rho_{M_{3}}(X)$ para todo $X \subseteq E$

iii. $\rho_{M_{2}}(X) \geq \rho_{M_{2}}(X)$ para todo $X \in \xi_{M_{2}}$

Notação: $M_{1} \preceq M_{3}$.

A relação $\leq$ é uma relação de ordem parcial sobre $M(E)$ q̨ue chamaremos de ordem fraca. O exemplo abaixo mostra que infelismente o conjunto ordenado $(M(E), \preceq)$ não \& um reticulado:

Easenplo 1.1. Considere os aeguintes matróides sobre o conjunto $E=\{a, b, c, d\}$
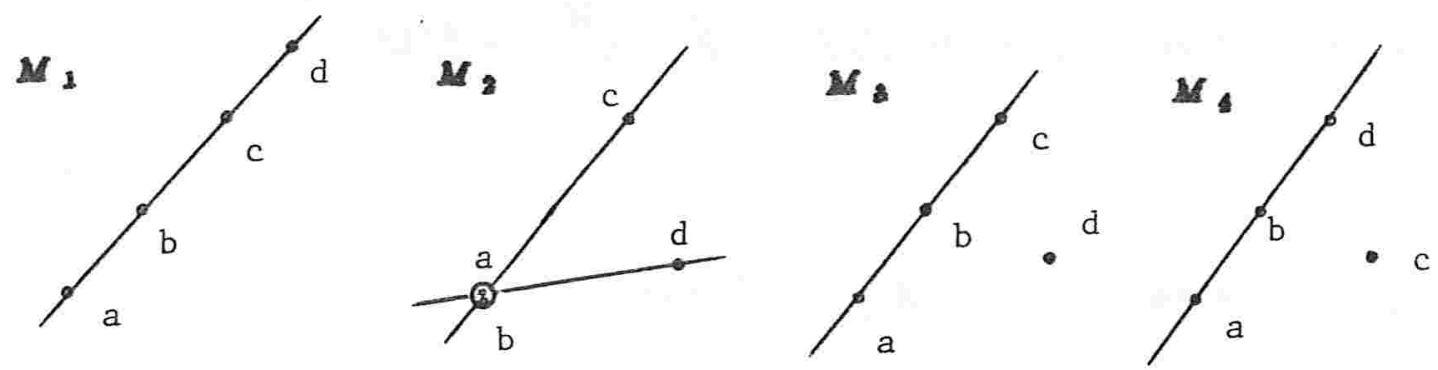

Mostra-se facilmente que $M_{1}, M_{3}$ cobrem $M_{3}, M_{4}$ e portanto $M_{3} \vee M_{4}$ e $M_{1} \wedge M_{2}$ nč̃o existem.

\section{EXTENSÕES}

Durante o restante do texto, salvo menção explicita em contrário, $P$ é um conjunto finito disjunto de $E$.

Uma extensão do matróide $M(E)$ sobre $E \cup P$ é um matróde $N(E \cup P)$ tal que $N \backslash P=N(\bar{E})=M(\bar{E})$. Notação: $M(E) \hookrightarrow N(E \cup P)$.

Se $P=\{x\}$, a extensão $N$ é dita pontual. Uma maneira bbvia de obter uma extensão pontual de $M$ é definir um matroide $N$ sobre $E \cup x$ cujas bases são da forma $B \cup x$ onde $B$ é base de $M$. 0 matróide $N$ assim definido será chamado de soma de um ponto a $M$. Note que nesse caso, $\rho N=\rho M+1$, e além disso, $N$ é a única extensão de $M$ com essa propriedade. Vamos então considerar apenas extensões pontuais $N$ de $M$ tais que $\rho N=\rho M$. Nesse caso, temos uma 
caracterização bastante interessante para as extensões pontuais. Dizemos que $\subseteq$ $F_{M}$ é un futro modular de $M$ se as seguintes condições estão satisfeitas:

(F1) Se $X \in \mathcal{J}, Y \in \mathcal{J}_{M} \in X \subseteq Y$ então $Y \in \mathcal{Z}$

(F2) Se $X, Y \in \mathcal{F}$ formam um par modular em $M$, igto é,

$$
\rho_{M}(X)+\rho_{M}(Y)=\rho_{M}(X \cup Y)+\rho_{M}(X \cap Y)
$$

então $X \cap Y \in \xi$

Não é difficil ver que se $N$ é uma extensão pontual de $M$ sobre $E \cup \mp$ então $F=$ $\left\{X \in \bar{f}_{M}: x \in \bar{X}^{N}\right\}$ é um filtro modular de $M$.

Teorema (Wxtensão Ponteal) Seja om filtro modular de M. Então

$$
\begin{aligned}
& \mathcal{I}_{N}=\{X \cup \tilde{X}: X \in \xi\} \cup\left(\mathcal{F}_{M}-\xi\right) \cup\left\{X \cup \mathscr{I}: X \in \mathcal{F}_{M}-\xi\right. \\
& X \text { Ião é coberto por elemento de } \overline{\}}
\end{aligned}
$$

é o conjunto dos fechados de uma extensão pontual $N$ de $M$ sobre $E \cup x$. Além disso, $J=\left\{X \in \bar{F}_{M}: x \in \bar{X}^{N}\right\}$.

Notação: $M(\bar{E}) \stackrel{g}{\longrightarrow} N(E \cup x)$ ou $N$ é a extersão pontual de $M$ sobre $\bar{E} \cup x$ pelo filtro modular $\$$.

Dada uma familia $\mathcal{G}$ de fechados de $M \circ$ filtro modular de $M$ gerado por $G$, denotado por $[\mathcal{G}]_{M}$ ou apenas $[9]$ se não houver perigo de ambiguidade, é a intersecção de todos os filtros modulares de $M$ que têm $g$ como subfamilia. Se $g$ é unitário, jato $\hat{e}, \mathcal{G}=\{A\}$ para algum $A$ fechado de $M,[G]$ é dito filtro principal gerado por $A$. $E$ fácil ver nesse caso que $[A]=\left\{X \in \mathcal{I}_{\mathbb{M}}: A \subseteq X\right\}$.

Diremas que uma sequência de filtros modulares de $M, f_{1}, f_{2}, \ldots, \xi_{n}$ é compativel, ou que $\xi_{1}, \xi_{2}, \ldots, \tilde{F}_{n}$ são futros modulares compativess se existe uma extensão $N$ de $M$ sobre $E \cup P, P=\left\{p_{1}, p_{2}, \ldots, p_{n}\right\}$, tal que

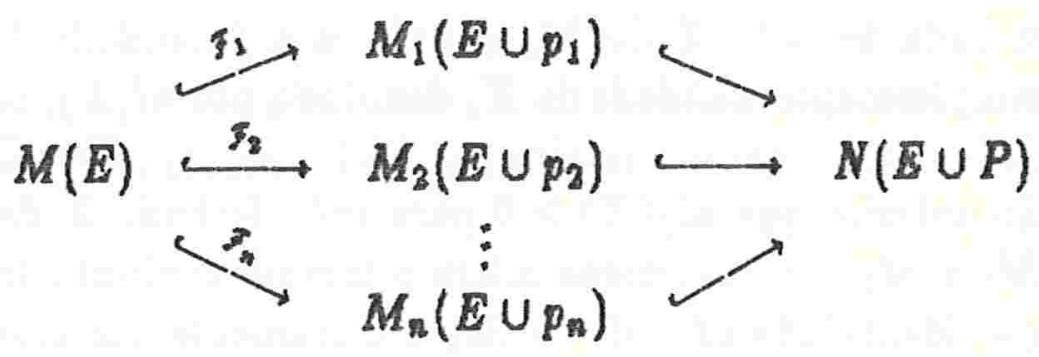

Em particular, se $n=2, N$ é chamada \&-eztensäo de $M$. 
Definimos agora o defeito de um par de fechados $X, Y$ de $M$ como sendo o natural $\rho_{M}(X)+\rho_{M}(Y)-\rho_{M}(X \cup Y)-\rho_{M}(X \cap Y)$ e denotamos esse número por $\operatorname{def}_{M}(X, Y)$.

\section{MORFISMOS FORTES}

Sejam $M_{1}, M_{2}$ matróides sobre os conjuntos $E_{1}, E_{2}$ respectivamente, e um novo elemento $0 \notin E_{1} \cup E_{2}$. Para cada $i=1,2$ considere $M_{i}^{\prime}$ a extensão de $M_{i}$ sobre $E_{i}$ U0 tal que 0 é laço de $M_{i}^{!}$. Um morfismo forte é uma função de $E_{1} \cup 0 \mathrm{em}$ $E_{2} \cup 0_{1}$ denotada por $f: M_{1} \rightarrow M_{2}$ cuja imagem contém o conjunto dos fechados de $M_{2}$, tal que

(MF1) $f(0)=0$

(MF2) Para todo fechado $X$ de $M_{2}^{\prime}, f^{-1}(X)$ é fechado de $M_{1}^{\prime}$

No que se segue, $f: M_{1} \rightarrow M_{2}$ já denotará uma função de $E_{1} \cup 0 \rightarrow E_{2} \cup 0$ tal que $f(0)=0$ e cuja imagem contém o conjunto dos fechados do matróide $M_{2}$.

Proposiçõo (Morifmo Porte 1) Sejam $M_{1}, M_{2}$ matróides sobre os conjuntos $E_{1}, E_{2}$ respectivamente. Dada $f: M_{1} \rightarrow M_{2}$, são equivalentes

i. fé mortismo forte

ii. Para todo $X \subseteq E_{1}$ temos que $f\left(\bar{X}^{M_{1}}\right) \subseteq \overline{f(X)}^{M_{2}}$

iii. Para todos $X, Y \subseteq \mathbb{E}_{1}$ tais que $Y \subseteq X$ temos que

$$
\rho_{M_{2}}(f(X))-\rho_{M_{2}}(f(Y)) \leq \rho_{M_{1}}(X)-\rho_{M_{2}}(Y)
$$

Para cada fechado $X$ de $M_{1}$ definimos a $f$-nulidade de $X$, denotada por $n l_{f}(X)$, ou simplesmente nalidade de $X$, denotada por $n l(X)$, caso nz̃o haja perigo de ambiguidade, como senco o inteiro $\rho_{M_{1}}(X)-\rho_{M_{2}}(f(X))$. Decorre do item iii. da proposição anterior que $n l_{f}(X) \geq 0$ para todo fechado $X$ de $M_{1}$.

Se $M_{1} \in M_{2}$ são matroides sobre o mesmo conjunto tais que $\mathcal{J}_{M_{2}} \subseteq \mathcal{I}_{M_{1}}$ então a função identidade id: $M_{1} \rightarrow M_{3}$ é claramente um morfismo forte. Nesse caso $M_{2}$ é chamado de quociente de $M_{1}$ cujo grae é o inteiro não negativo $\rho M_{1}-$ 
$\rho M_{2}$, denotado por $\operatorname{gr}\left(M_{1} \rightarrow M_{3}\right)$. Se $\operatorname{gr}\left(M_{1} \rightarrow M_{2}\right)=1$ então $M_{2}$ é chamado grociente elementar de $M_{1}$.

Proponição (Morimmo Forte 2) Sejam $M_{1}, M_{2}$ matróides sobre o mesmo conjunto $E$ tais que $M_{2}$ é quociente de $M_{1}$. Então para todo par de fechados de $M_{1}$
i. $n l(X) \leq \operatorname{gr}\left(M_{1} \rightarrow M_{2}\right)$
ii. $\operatorname{def}_{M_{1}}(X, Y) \geq \operatorname{de}_{M_{2}}(X, Y)$
iii. $n l(X)+n l(Y)=n l(X \cup Y)+n l(X \cap Y)+\operatorname{def}_{M_{1}}(X, Y)-\operatorname{def}_{M_{2}}(X, Y)$
iv. $X, Y$ é par modular em $M_{l} \Leftrightarrow n l(X)+n l(Y)=n l(X \cup Y)+n l(X \cap Y)$ 


\section{Capítulo I}

\section{Ultra-Compatibilidade}

Apresentamos aqui uma condição suficiente natural, a ultra-compatibilidade, para que uma sequência finita de filtros modulares de um matróide seja compativel. Além disso, daremos caracterisaçōes simples para a extensão desse matróide determinada por uma sequência de flltros modulares ultra-compativeis. Como exemplo de tais extensões podemos citar as extensões pontuais e as principais.

O conceito de ultra-compatibilidade é introdusido na sua forma mais natural através da noção de sequência ultra-compatível. Na Seção 1, veremos que o conceito aplica-se à familia de filtros e não depende de que particulas sequência seus membros são colocados.

A segunda seção é dedicada ao estudo das extensões lisas de um matróide. Cada extensão desse tipo é unicamente determir ada por uma sequência de filtros modulares ultra-compatfveis. Uma breve análise da relação existente entre um determinado morismo forte e a ultra-compatibilidade também será feita no final dessa seção. 


\section{A ULTRA-COMPATIBILIDADE IMPLICANDO}

COMPATIBILIDADE

Seja $N$ uma extensão de $M$ e $q$ um filtro modular de $M$. Chamaremos de fecho de $\xi$ em $N$ o seguinte filtro modular de $N$,

$$
\left[\eta_{N}=\bigcap\left\{\xi^{\prime} \text { sltro modular de } N:\left\{\bar{X}^{N}: X \in \xi\right\} \subseteq \xi^{\prime}\right\}\right.
$$

Proposição 1.1. Sejam $f_{1}, f_{2}, \ldots, \eta_{n}$ fltros modulares de $M$ e $P=\left\{p_{1}, p_{2}, \ldots, p_{n}\right\}$. Considere também o seguinte diagrama de extensōes pontuais:

$$
M(E) \stackrel{\xi_{1}}{\longrightarrow} M_{1}\left(E \cup p_{1}\right) \stackrel{\left[\tilde{F}_{2}\right] M_{1}}{\longrightarrow} M_{2}\left(E \cup p_{1} \cup p_{2}\right) \stackrel{\left|F_{3}\right| M_{3}}{\longrightarrow} \ldots
$$

Então são equivalentes:

i. Para cada $i=2,3, \ldots, n$ o conjunto dos elementos minimais por inclusão de $\left[\boldsymbol{f}_{i}\right]_{M_{i-1}}$ coincide com o conjunto dos fechos em $M_{i-1}$ dos elementos minimais de $\tilde{f}_{i}$

ii. Para cada $i=2,3, \ldots, n$, se $X \in\left[\tilde{q}_{i}\right]_{M_{i-1}}$ então $X \cap E \in \bar{\xi}_{i}$

Prova: Verifiquemos que $i . \Rightarrow$ ii.. Fixemos $i \in\{2,3, \ldots, n\}$. Seja $X \in\left|\boldsymbol{f}_{i}\right|_{M_{i-2}}$; então existe $Y$ minimal em $\left|\tilde{f}_{i}\right|_{M_{i-3}}$ tal que $Y \subseteq X$. Pelo item $i$., existe $Y_{0} \in \tilde{f}_{i}$ tal que ${\overline{Y_{0}}}^{M_{i-1}}=Y$. Logo $Y_{0} \subseteq Y \cap E \subseteq X \cap E$, donde $X \cap E \in \bar{f}_{i}$.

Mostremos agora que $\ddot{x} \Rightarrow$ i. Fixemos $i \in\{2,3, \ldots, n\}$. Na realidade, podemos substituir ii. pela seguinte condição:

$$
\text { para todo } X \in \bar{I}_{M_{i-1}}, \quad X \in\left[\left.\tilde{F}_{i}\right|_{M_{i-1}} \Leftrightarrow X \cap E \in \tilde{f}_{i}\right.
$$

já que a outra implicação é trivial. Seja $X$ minimal em $\left|\bar{\eta}_{i}\right|_{M_{i-1}}$. Por $i i_{\text {. }} X \cap \mathbb{E} \in \bar{\xi}_{i}$. Tome $Y$ minimal em $f_{i}$ tal que $Y \subseteq X f_{1} E$. Então $\bar{Y}^{M_{i-1}} \subseteq \overline{X \cap E}^{M_{i-1}} \subseteq X$ e pela minimalidade de $X$ resulta que $\bar{Y}^{M_{i-1}}=\overline{X \cap E}^{M_{i-1}}=\bar{X}$ e $X \cap \bar{E}$ é minimal em $\boldsymbol{f}_{i}$. De outro lado, seja $Y$ minimal em $\boldsymbol{\xi}_{i}$. Temos que $\bar{Y}^{M_{i-1}} \in\left[\boldsymbol{f}_{i}\right]_{M_{i-1}}$. Tome $X$ minimal em $\left[\tilde{f}_{i}\right]_{M_{i-1}}$ tal que $X \subseteq \bar{Y}^{M_{i-1}}$. Por is., $X \cap E \in f_{i}$ e como 
$X \cap E \subseteq \bar{Y}^{M_{i-1}} \cap E=Y$, pela minimalidade de $Y, X \cap E=Y$. Logo $\bar{Y}^{M_{i-1}}=$ $\overline{X \cap E^{M_{i-1}}} \subseteq X \subseteq \bar{Y}^{M_{i-1}}$ e portanto $X=\bar{Y}^{M_{i-1}}$

Dada uma sequência $\bar{\xi}_{1}, \xi_{2}, \ldots, \xi_{n}$ de filtros modnlares de $M$, diremos que ela é uma sequéncia ultra-compatínel de $M$ se uma das condições equivalentes da Proposição 1.1. É satisfeita.

Note que ae $n=1$ a sequência \& trivialmente ultra-compativel. Combinando essa observação e o próximo resultado, chegamos à conclusão de que toda sequência de filtros principais de um mastóide é ultra-compativel.

Proposição 1.2. Seja $\xi_{1}, \xi_{2}, \ldots, \xi_{n}$ uma sequência ultra-compatfvel de $M$ e $\xi$ um

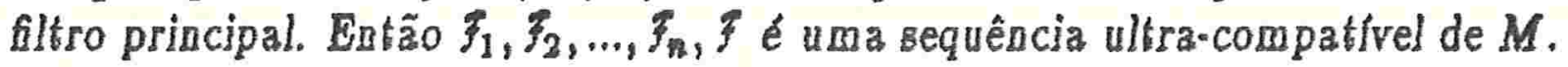

Prova: Considere o seguinte diagrama de extensões pontuais:

$$
\begin{aligned}
M(E) \stackrel{I_{1}}{\longrightarrow} & M_{1}\left(E \cup p_{1}\right) \stackrel{\left[\tilde{F}_{2}\right]_{M_{2}}}{\longrightarrow} \ldots \\
& \stackrel{\left[\xi_{n}\right]_{M_{n-1}}}{\longrightarrow} M_{n}\left(E \cup\left\{p_{1}, p_{2}, \ldots, p_{n}\right\}\right) \stackrel{[]_{B_{n}}}{\longrightarrow} N(E \cup P)
\end{aligned}
$$

onde $P=\left\{p_{1}, p_{2}, \ldots, p_{n}, p\right\}$. Como $\xi_{1}, \xi_{2}, \ldots, \xi_{n}$ é ultra-compatível, para que $\xi_{1}, \Phi_{3}, \ldots, \Phi_{n}, \xi$ também seja, basta mostrarmos que se $X \in|\xi|_{M_{n}}$ então $X \cap E \in \bar{\xi}$. Seja $X \in\left[\left.F\right|_{M_{n}}\right.$. Da hipótese, $F$ é filtro principal, logo existe $A \subseteq E$ tal que $f=\left\{Y \in f_{M}: A \subseteq Y\right\}$. Portanto $A \subseteq X$, donde $A \subseteq X \cap E \in f_{M}, 0$ que mostra que $X \cap E \in \mathcal{F}$.

A Proposição 1.4. a seguir nos fornece uma caracterização mais natural para a ultra-compatibilidade. Antes de enunció-la, um pequeno Lema se faz necessário.

Lema 1.8. Sejam $X, Y$ fechados de $M$ e $N$ a extensão pontual de $M$ sobre $E \cup p$ pelo filtro modular F. Então,

$$
\operatorname{def}_{N}\left(\bar{X}^{N}, \bar{Y}^{N}\right)= \begin{cases}\operatorname{def}_{M}(X, Y)-1, & \text { se } X, Y \in \mathcal{Y} \text { e } X \cap Y \notin \bar{Y} \\ \operatorname{def}_{M}(X, Y), & \text { caso contrário }\end{cases}
$$


Prova: Considere inicialmente o caso em que $X, Y \in \mathcal{F}$ e $X \cap Y \notin \xi$. Assim, $X \cup Y \in \bar{z}$

$$
\begin{aligned}
\rho_{N}\left(\overline{\boldsymbol{X}}^{N}\right) & =\rho_{M}(\boldsymbol{X}) \\
\rho_{N}\left(\overline{\boldsymbol{Y}}^{N}\right) & =\rho_{M}(\boldsymbol{Y}) \\
\rho_{N}\left(\overline{\boldsymbol{X}}^{N} \cup \overline{\boldsymbol{Y}}^{N}\right) & =\rho_{N}\left(\overline{\boldsymbol{X} \cup \boldsymbol{Y}^{N}}\right)=\rho_{M}(\boldsymbol{X} \cup Y) \\
\rho_{N}\left(\overline{\boldsymbol{X}}^{N} \cap \overline{\boldsymbol{Y}}^{N}\right) & =\rho_{N}((\boldsymbol{X} \cup p) \cap(\boldsymbol{Y} \cup \varphi))=\rho_{N}((\boldsymbol{X} \cap \boldsymbol{Y}) \cup p)=\rho_{M}(\boldsymbol{X} \cap \boldsymbol{Y})+\mathbb{I}
\end{aligned}
$$

Logo, $\operatorname{de} f_{N}\left(\bar{X}^{N}, \bar{Y}^{N}\right)=\operatorname{def} f_{M}(X, Y)-1$.

De outro lado, no caso complementar, é possivel provar de maneira aemelhante que de $f_{N}\left(\bar{X}^{N}, \bar{Y}^{N}\right)=\operatorname{def}(X, Y)$.

Proposição 1.1. Sejam $\xi_{1}, \xi_{2}, \ldots, \xi_{n}$ filtros modulares de $M$. São equivalentes:

i. $\Phi_{1}, \Phi_{2}, \ldots, \xi_{n}$ é sequéncia ultra-compativel

ii. $\xi_{1}, \xi_{2} \ldots, \xi_{n-1}$ sequência ultra-compatfvel e para cada $I \subseteq\{1,2, \ldots$, $n-1\}$, se $X, Y \in\left(\bigcap_{i \in I} \tilde{f}_{i}\right) \cap \bar{\gamma}_{m} \operatorname{com} \operatorname{def}_{M}(X, Y)=|I|$ então existe $k \in I \cup n$ tal que $X \cap Y \in F_{k}$

Prova: Considere o seguinte diagrama de extensões pontuais:

$$
\begin{aligned}
M(E)=M_{0}(E) \stackrel{\xi_{1}}{\longrightarrow} M_{1}\left(E \cup p_{1}\right) & \stackrel{\left[\xi_{2}\right]_{M_{1}}}{\longrightarrow} M_{2}\left(E \cup p_{1} \cup p_{2}\right) \\
& \stackrel{\left[\xi_{3}\right]_{N_{3}}}{\longrightarrow} \ldots \stackrel{\left[\xi_{n}\right]_{N_{n-1}}}{\longrightarrow} M_{n}(E \cup P)
\end{aligned}
$$

onde $P=\left\{p_{1}, p_{2}, \ldots, p_{n}\right\}$.

Verifiquemos que $i .7$ is.. Seja $I \subseteq\{1,2, \ldots, n-1\}$ tal que existem $X$, $Y \in\left(\bigcap_{i \in I} f_{i}\right) \cap F_{n}$ com def $f_{M}(X, Y)=|I|$. Se existe $k \in I$ tal que $X \cap Y \in F_{k}$, não temos nada a provar. Suponha então que para todo $k \in I, X \cap Y \notin f_{k}$. Como $\xi_{1}, \xi_{3}, \ldots, \xi_{n-1}$ são filtros modulares ultra-compatíveis, temos que $\overline{X \cap Y}{ }^{M_{k-1}} \notin$ $\left[f_{k}\right]_{M_{k-1}}$ para $k \in I$. Usando o Lema anterior $|I|$ veges obtemos $\operatorname{de} f_{M}(X, Y) \geq$ $\operatorname{def}_{M_{n-1}}\left(\bar{X}^{M_{n-1}}, \bar{Y}^{M_{n-1}}\right)+|I|$. Logo def $M_{n-1}\left(\bar{X}^{M_{n-1}}, \bar{Y}^{M_{n-1}}\right)=0,0$ que significa que $\bar{X}^{M_{n-1}}, \bar{Y}^{M_{n-1}}$ é um pur modular em $M_{n-1}$. Como $\bar{X}^{M_{n-1}}, \bar{Y}^{M_{n-1}} \in$ $\left[\left.\tilde{f}_{n}\right|_{M_{n-1}}\right.$, temos que $\bar{X}^{M_{n-1}} \cap \bar{Y}^{M_{n-1}} \in\left[\tilde{F}_{n}\right]_{M_{n-1}}$, e, usando a hipótese de ultracompatibilidade temos que $X \cap Y \in \bar{I}_{n}$.

Verifiquemos agora que $\ddot{u} . \Rightarrow i_{\text {. }}$ Suponha por absurdo que $\xi_{1}, \xi_{2}, \ldots, \xi_{n}$ não é ultra-compatível. Como $\xi_{1}, \xi_{2}, \ldots, \xi_{n-1}$ é ultra-compativel por is., essa suposição 
implica na existência de $X, Y \in \mathcal{I}_{n} \operatorname{com} X \cap Y \notin F_{n}$, def $f_{M}(X, Y)=d$ e $\bar{X}^{M_{n-1}}$, $\bar{Y}^{M_{n-1}}$ par modular em $M_{n-1}$, isto é, de $f_{M_{n-1}}\left(\bar{X}^{M_{n-1}}, \bar{Y}^{M_{n-1}}\right)=0$. Usando o Lema 1.3., conclui-se que existe $I \subseteq\{1,2, \ldots, n-1\}$ tal que $|I|=d, X, Y \in \bigcap_{i \in I} \boldsymbol{\gamma}_{i}$ e $X \cap Y \notin \xi_{k}$ para $k \in I$ o que contradis $i i_{.}$. Logo, $\eta_{1}, \eta_{3}, \ldots, I_{n}$ é uma sequência ultra-compativel de $M$.

Note que ii. é equivalente a:

ii'. Para cada $I \subseteq\{1,2, \ldots, n-1\}$, se $X, Y \in \bigcap_{i \in I} \xi_{i}$ e def $M(X, Y)=|I|-1$ então existe $k \in I$ tal que $X \cap Y \in \mathcal{F}_{k}$

que não depende da indexação dos filtros $\xi_{1}, \xi_{2}, \ldots, \xi_{n}$.

Corolário 1.5. Seja W uma familia de filtros modulares de $M$. São equivalentes:

i. Existe uma indexação $\xi_{1}, \xi_{2}, \ldots, \xi_{n}$ de $W$ tal que essa sequência é ultracompativel

ii. Para toda indexação $F_{1}, F_{2}, \ldots, I_{n}$ de $W$ essa requência é ultra-compatível

Podemos então diser que os filtros modulares $\xi_{1}, \xi_{2}, \ldots, \eta_{n}$ são ultra-compatíveis se a sequência $\xi_{1}, \xi_{3}, \ldots, \xi_{n}$ é ultra-compativel. 0 corolário anterior nos garante que esse conceito é bem definido. Note bem que o mesmo flltro pode ocorrer repetidas vezes na famillia. A Proposição anterior permite obter, de uma maneira mais natural, o seguinte resultado de Cordovil [Co2]:

Corolário 1.6. Sejam $f_{1}, \xi_{2}, \ldots, \xi_{n}$ conjuntos de fechados de $M$ e $P=\left\{p_{1}, p_{2}, \ldots\right.$, $\left.p_{n}\right\}$. São equivalentes:

i. $\xi_{1}, \xi_{3}, \ldots, \xi_{n}$ são filtros modulares ultra-compativeis de $M$

ii. Considerando a segujnte função $\partial$ sobre ${ }_{M}$ no conjunto das partes de $P$ definida por

$$
\partial(X)=\left\{p_{i}: X \in \bar{\xi}_{i}\right\}
$$

para todos $X, Y \in \mathcal{J}_{M}$ temos

(a) Se $X \subseteq Y$ então $\partial(X) \subseteq \partial(Y)$ 


$$
\text { (b) } \operatorname{def}_{M}(X, Y) \geq|(\partial(X) \cap \partial(Y))-\partial(X \cap Y)|
$$

Prova: Mostremos que i. $\Rightarrow$ ii.. 0 item (a) é trivial já que $\bar{\eta}_{1}, \xi_{2}, \ldots, \xi_{n}$ são filtros modulares. Suponhamos que existem $X, Y \in I_{M}$ tais que de $f_{M}(X, Y)=d<$ $|(\partial(\boldsymbol{X}) \cap \partial(\boldsymbol{Y}))-\partial(\boldsymbol{X} \cap \boldsymbol{Y})|$. Entäo existe $I \subseteq\{1,2, \ldots, n\}$ tais que $X, Y \in \bigcap_{i \in I} \boldsymbol{f}_{i}$ e $X \cap Y \notin \bigcup_{i \in I} \bar{z}_{i}$ o que contraria a ultra-compatibilidade de i.. Portanto temos (b).

Verifiquemos que is $\Rightarrow i$. Inicialmente, $\xi_{1}, \xi_{2}, \ldots, \xi_{n}$ são filtros modulares de $M$ pois $\ddot{3}$.(a) implica que para todos $X, Y \in \mathcal{I}_{M}$, se $X \subseteq Y$ e $X \in \mathcal{J}_{i}$ então $Y \in \mathcal{F}_{i}$, e $X \cap Y \in \mathcal{F}_{i}$. Sejam $X, Y \in \mathcal{F}_{M}$ e $I \subseteq\{1,2, \ldots, n\}$ tais que $\operatorname{def}_{M}(X, Y)=|I|-1$ e $X, Y \in \bigcap_{i \in I} \mathcal{F}_{i}$. Então de $\ddot{i}$ (b) concluimos que $X \cap Y \in \mathcal{P}_{k}$ para algum $k \in I$. Logo, $\mathcal{f}_{1}, \mathcal{F}_{3}, \ldots, \mathcal{F}_{n}$ são filtros modulares ultra-compativeis de $M$.

Corolario 1.7. Seja $\bar{l}$ um fltro modular de $M$. Uma sequência $\xi_{1}, \xi_{2}, \ldots, \xi_{n}$ de $n$ cópias de $\xi$ é ultra-compativel se e somente se $\xi$ é principal ou $n \leq$ $\min \left\{\operatorname{def} f_{M}(X, Y): X, Y \in \mathcal{F}, X \cap Y \notin \mathcal{F}\right\}$.

O restante da seção é dedicado a dełerminar a relaçäo existente entre a ultra-compatibilidade e a compatibilidade de filtros modulares. 0 próximo Teorema, em particular, servirá de inspiração para a definição de extensão lisa, tópico da próxima seçãa.

Teorema 1.8. Seja $N$ uma extensão de $M$ sobre $E \cup P$ tal que $|P|=$ n. São equivalentes:

i. Existe uma indexação $P=\left\{p_{1}, p_{2}, \ldots, p_{n}\right\}$ tal que para $i=2,3, \ldots, n$ temos para todo $X \subseteq E \cup\left\{p_{1}, p_{2}, \ldots, p_{i-1}\right\}$ :

$$
p_{i} \in \bar{X}^{N} \Leftrightarrow p_{i} \in{\overline{\bar{X}^{N}} \cap \mathbb{E}}^{N}
$$

ii. Para todo $X \subseteq E \cup P$ temos $\bar{X}^{N}=\overline{\bar{X}}^{N} \cap \bar{E} \cup X$ 
Prova: A implicação $\ddot{i} . \Rightarrow$ i. é trivial. Mostremos que $i . \Rightarrow$ ii. por indução sobre $n$. $A$ base da indução é dada por $n=0$. Nesse caso $N=M$ e ii. é imediato. Suponha que $n>0$ e sejam $p=p_{n}, N^{\prime}=N \backslash p$ e $X \subseteq E \cup P$. Basta então mostrarmos que $\bar{X}^{N} \subseteq \bar{X}^{N} \cap \bar{E} \cup X$ para verificarmos o item î., já que a inclusão contrária é clara. Temos dois casos a considerar:

Caso 1: $p \notin X$

Por hipótese de indução temos que $\bar{X}^{N^{\prime}}={\overline{\bar{X}^{N \prime}}}^{N^{\prime}}{ }^{N^{\prime}} \cup X$. Se $p \notin \bar{X}^{N}$ então

$$
\bar{X}^{N}=\bar{X}^{N^{\prime}}={\overline{\bar{X}^{N^{\prime}} \cap E}}^{N^{\prime}} \cup X \subseteq{\overline{\bar{X}^{N}} \cap \bar{E}}^{N} \cup X
$$

Por outro lado, se $p \in \bar{X}^{N}$ então

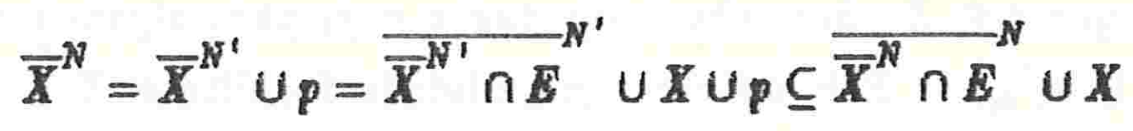

Caso 2: $p \in X$

Por hipótese de indução temos que

$$
\overline{X-p}^{N^{\prime}}=\overline{\overline{X-p}}^{N^{\prime}} \cap E^{N^{\prime}} \cup(X-p) \subseteq{\overline{\bar{X}^{N}}}^{N} \mathrm{E} \cup X
$$

Se $p \in \overline{X-p}^{N}$ então

$$
\bar{X}^{N}=\overline{X-p}^{N} \subseteq \overline{X-p}^{N^{\prime}} \cup p \subseteq{\overline{\bar{X}^{N}} \cap E}^{N} \cup X
$$

Suponha então que $p \notin \overline{X-p}^{N}$. Se $\bar{X}^{N}-\overline{X-p}^{N}=p$ então $\bar{X}^{N} \subseteq$ $\overline{\bar{X}}^{N} \cap \bar{E} \cup X$ pois $g \in X$. Podemos supor então que existe $q \neq p$ tal que $p, q \in \bar{X}^{N}-\bar{X}-p^{N} . \log \rho p \in \overline{(X-p) \cup q}_{N}^{N}$ e $\bar{X}^{N}=\overline{(X-p) \cup q}^{N}$. Por

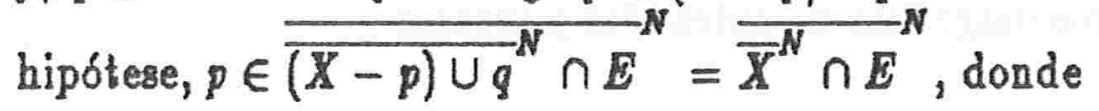

$$
\bar{X}^{N}={\overline{\left(\bar{X}^{N} \cap E\right) \cup(X-p)}}^{N}={\overline{\left(\bar{X}^{N} \cap E\right) \cup(X-p)}}^{N^{\prime}} \cup p
$$

Portanto usandn a hipótese de indução

$$
\begin{aligned}
\bar{X}^{N} & =\overline{\left(\bar{X}^{N} \cap E\right) \cup(X-p)}{ }^{N^{\prime}} \cap N^{\prime} \\
& \subseteq \overline{\bar{X}}^{N} \cap E \cup\left(\bar{X}^{N} \cap E\right) \cup(X-p) \cup p
\end{aligned}
$$


Lema 1.9. Sejam $\xi_{1}, \xi_{2}, \ldots, \xi_{n}$ fltros modulares ultra-compatfveis de $M$ e considere o respectivo diagrama de extensões pontuais:

$$
M(E) \stackrel{\xi_{1}}{\longrightarrow} M_{1}\left(E \cup p_{1}\right) \stackrel{\left[\beta_{3}\right]_{M_{1}}}{\longrightarrow} \ldots \stackrel{\left[g_{n} \mid M_{n-2}\right.}{\longrightarrow} M_{n}(E \cup P)
$$

onde $P=\left\{p_{1}, p_{2}, \ldots, p_{n}\right\}$. Então para todo fectado $X$ de $M$ temos que

$$
\bar{X}^{M_{n}}=X \cup\left\{p_{i} \in P: X \in \bar{F}_{i}\right\}
$$

Prova: Seja $X$ fechado de $M$. A inclusão $\bar{X}^{M_{n}} \supseteq X \cup\left\{p_{i} \in P: X \in f_{i}\right\}$ é facilmente verificada. Mostremos então que vale a inclusão contrária. Sabemos que $\boldsymbol{X}=\bar{X}^{M}=\bar{X}^{M_{n}}-\left\{p_{1}, p_{2}, \ldots, p_{n}\right\}=\bar{X}^{M_{n}} \cap E$. Seja $x \in \bar{X}^{M_{n}}$. Se $z \in E$ é claro que $x \in X$. Suponha então que $x=p_{i}$ para algum $i=1,2, \ldots, n$. Então $\bar{X}^{M_{i-1}} \in\left[F_{i}\right]_{M_{i-1}}$. Pela ultra-compatibilidade dos filtros, $\bar{X}^{M_{i-1}} \cap E \in \mathcal{J}_{i}$. Mas

$$
\bar{X}^{M_{i-1}} \cap E=\left(\bar{X}^{M_{n}} \cap\left(E \cup\left\{p_{1}, p_{2}, \ldots, p_{i-1}\right\}\right)\right) \cap E=\bar{X}^{M_{n}} \cap E=X
$$

Logo $X \in \xi_{i}$, donde $x \in\left\{p_{i} \in P: X \in \bar{\eta}_{i}\right\}$.

Proposição 1.10. Sejam $\eta_{1}, \tilde{F}_{2}, \ldots, \eta_{n}$ filtros modulares ultra-compativeis de $M$ e considere o respectivo diagrama de extensões pontuais

$$
M(E) \stackrel{g_{1}}{\longrightarrow} M_{1}\left(\bar{E} \cup p_{1}\right) \stackrel{\left.\mid \tilde{F}_{2}\right]_{M_{1}}}{\longrightarrow} \ldots \stackrel{\left[F_{n}\right]_{M_{n-1}}}{\longrightarrow} M_{n}(E \cup P)
$$

onde $P=\left\{p_{1}, p_{2}, \ldots, p_{n}\right\}$. Considere uma outra indexaça $\tilde{i}_{i_{1}}, \tilde{q}_{i_{2}}, \ldots, \tilde{f}_{i_{n}}$ dos fltros acima e o respectivo diagrama de extensões pontuais

$$
M(E) \stackrel{F_{i_{3}}}{\longrightarrow} N_{1}\left(E \cup p_{i_{1}}\right) \stackrel{\left|\tilde{f}_{i_{1}}\right| N_{1}}{\longrightarrow} \ldots \stackrel{\left|\tilde{f}_{i_{A}}\right| N_{n-1}}{\longrightarrow} N_{n}(E \cup P)
$$

Então $M_{n}=N_{n}$.

Prova: Usando a ultra-compatibilidade de $\xi_{1}, \xi_{2}, \ldots, \xi_{n}$, mostremos que $M_{n}$ satisfaz a condição $i$. do Teorema 1.7.. Tome a indexação $P=\left\{p_{1}, p_{2}, \ldots, p_{n}\right\}$ e fixemos $i \in\{1,2, \ldots, n\}$. Considere $X \subseteq E \cup\left\{p_{1}, p_{2}, \ldots, p_{i-1}\right\}$. Se $p_{i} \in{\overline{\bar{X}^{M_{n}} \cap E}}^{M_{n}}$ é 
claro que $p_{i} \in \bar{X}^{M_{n}}$ já que ${\overline{X^{M_{n}}} \cap E}^{M_{n}} \subseteq \bar{X}^{M_{n}}$. Suponha por outro lado que $p_{i} \in \bar{X}^{M_{n}}$. Portanto $\bar{X}^{M_{i-1}} \in\left[\bar{f}_{i}\right]_{M_{i-1}}$ e pela ultra-compatibilidade dos filtros, $\bar{X}^{M_{i-1}} \cap E \in \bar{\xi}_{i}$ L Logo

$$
p_{i} \in{\overline{X^{M_{i-1}} \cap E}}^{M_{n}} \subseteq{\overline{\bar{X}^{M_{n}} \cap E}}^{M_{n}}
$$

Com isso acabamos de mostrar o item i. do Teorema anterior e portanto vale o item ii., isto e, para todo $X \subseteq E \cup P$ temos $\bar{X}^{M_{n}}={\overline{X^{M_{n}}} \cap E}^{M_{n}} \cup X$. Analogamente prova-se que para todo $X \subseteq E \cup P$ temos $\bar{X}^{N_{n}}={\overline{X^{N}}}^{N_{n}} \bar{E}^{N_{n}} \cup X$, já que a ultra-compatibilidade independe da indexação dos âltros. Mas pelo Lema anterior concluímos que para todo $X \subseteq E \cup P, \bar{X}^{N_{n}} \cap E{ }^{N_{n}}=\overline{\bar{X}}^{M_{n}} \cap E^{M_{n}}$ e porsanto $\bar{X}^{N_{n}}=\bar{X}^{M_{n}}$, donde $M_{n}=N_{n}$.

Como consequência imediata dessa Proposição temos finalmente que:

Corolário 1.11. Se $\eta_{1}, \pi_{2}, \ldots, \xi_{n}$ são filtros modulares ultra-compat́fvejs de $M$ então $\tilde{I}_{1}, \bar{\tau}_{3}, \ldots, \bar{\eta}_{\mathrm{n}}$ são filtros modulares compatfveis de $M$.

O próximo exemplo mostra que a ultra-compatibilidade não é condição necessária para a compatibilidade.

Exemplo 1.12. Seja $E=\{1,3, \ldots, 9\}$ e $M(E)=\mathcal{L}_{4}(E)$. Considere os seguintes filtros modulares de $M$ :

$$
\begin{aligned}
\xi_{1}=\xi_{2} & =[123,456,789] \\
\xi_{3} & =[123,456]
\end{aligned}
$$

e o seguinte diagrama de extensões pontuais:

$$
M(E) \stackrel{p_{s}}{\longrightarrow} M_{1}\left(E \cup p_{3}\right) \stackrel{\left[\xi_{3}\right]_{M_{1}}}{\longrightarrow} M_{2}\left(E \cup p_{1} \cup p_{3}\right) \stackrel{\left.\mid \xi_{3}\right]_{M_{3}}}{\longrightarrow} M_{3}\left(E \cup p_{1} \cup p_{2} \cup p_{3}\right)
$$




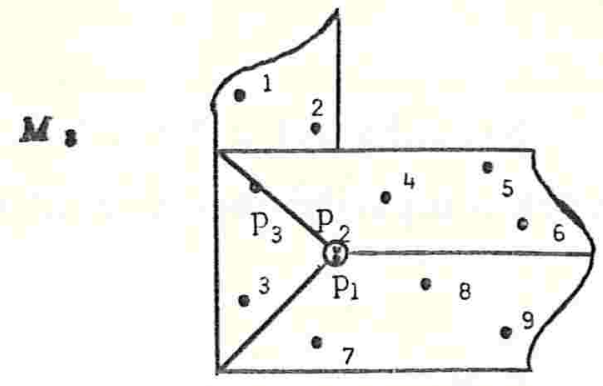

Figura 1

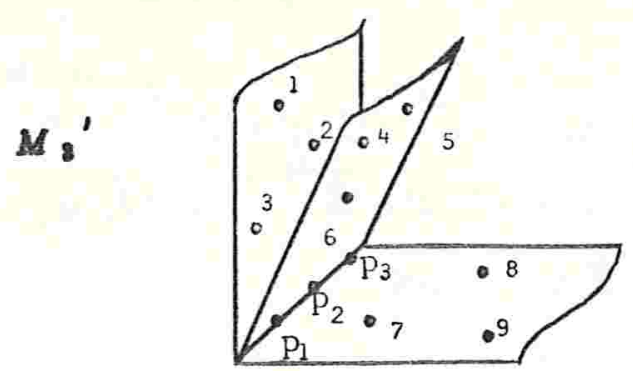

Tigura 2

Veja Figura 1.

É fácil ver que $\tilde{f}_{1}, \tilde{F}_{2}, \bar{J}_{3}$ são compatfveis. Congidere agora o seguinte diagrama de extensōes pontuais:

$M(E) \stackrel{\bar{F}_{1}}{\longrightarrow} M_{1}^{\prime}\left(E \cup p_{1}\right) \stackrel{\left[\left.\tilde{f}_{9}\right|_{M_{1}^{\prime}}\right.}{\longrightarrow} M_{2}^{\prime}\left(E \cup p_{1} \cup p_{2}\right) \stackrel{\left|\boldsymbol{s}_{3}\right|_{M_{3}^{\prime}}}{\longrightarrow} M_{3}^{\prime}\left(E \cup p_{1} \cup p_{2} \cup p_{3}\right)$

Veja Figura 2.

Como $M_{3} \neq M_{3}^{\prime}$ temos que $\tilde{F}_{1}, \mathcal{F}_{2}, \mathcal{F}_{3}$ ถão são ultra-compativeis.

\section{EXTENSÕES LISAS}

Seja $N$ uma extensz̃o de $M$ sobre $\bar{B} \cup P$. Diremos que $N$ é nma eatensão lisa de $M$ se para todo $X \subseteq E \cup P$ temos $\bar{X}^{N}=\overline{\bar{X}}^{N} \cap E \cup \cup$.

Proposiçূ̄o 2.1. Existe uma bijeção entre a famflia das sequércias ultra-compatreis de fltros modulares de $M$ e a famflia das extensóes lisas de $M$.

Prova: Considere a função que leva $\Phi_{1}, \Phi_{2}, \ldots, \Phi_{n}$ fltros modulares ultra-compatíveis de $M$ na extensão $N$ de $M$ sobre $E \cup P, P=\left\{p_{1}, p_{2}, \ldots, p_{n}\right\}$, dada pelo diagrama abaixo:

$$
M(E) \stackrel{\bar{F}_{1}}{\longrightarrow} M_{1}\left(E \cup p_{1}\right) \stackrel{\left[F_{2}\right]_{M_{1}}}{\longrightarrow} \ldots \stackrel{\left[\left.f_{n}\right|_{M_{n-1}}\right.}{\longrightarrow} M_{n}(E \cup P)=N(E \cup P)
$$

A função está bem definida pois $N$ independe da indexação dos filtros e $N$ é realmente uma extensão lisa de $M$. Suponhamos que existam duas sequências, $\xi_{1}, F_{2}, \ldots, \xi_{n} \in g_{1}, \mathfrak{I}_{2}, \ldots, \mathfrak{g}_{n}$, de filtros modulares ultra-compatíveis de $M$ levadas 
pela função acima à mesma extensão lisa $N$ de $M$. Mas então para cada $i=$ $1,2, \ldots, n$

$$
\xi_{i}=\left\{X \in \bar{I}_{M}: p_{i} \in \bar{X}^{N}\right\}=g_{i}
$$

o que mostra que a função é injetora. Sejam $N$ uma extensão lisa de $M$ sobre $E \cup\left\{p_{1}, p_{2}, \ldots, p_{n}\right\}$ e os filtros modulares $f_{i}=\left\{X \in \xi_{M}: p_{i} \in \bar{X}^{N}\right\}, i=1,2, \ldots, n$. Provemos que $\xi_{1}, \xi_{2}, \ldots, \xi_{n}$ são ultra-compativeis. Construa um diagrama como em $(*)$ e seja $X \in\left[\xi_{i}\right]_{M_{i-1}}$ para $i \in\{1,2, \ldots, n\}$ f́xo. Então $p_{i} \in \bar{X}^{N}$ e portanto $p_{i} \in \overline{\bar{X}}^{N} \cap E$, logo, pela definição dos $\xi_{i}^{\prime} s, \bar{X}^{N} \cap E \in \xi_{i}$. Mas $\bar{X}^{N} \cap E=$ $\bar{X}^{M_{i-1}} \cap E$. Mostramos assim que a função definida acima é também sobrejetora, estabelecendo-se a bijeção mencionada.

Passemos a caracteriear as extensões lisas.

Teorema 2.2. Seja $N$ uma extensão de $M$ sobre $E \cup P$. Então são equivalentes:

i. $N$ é uma extensão lisa de $M$

i8. Para todo $X \subseteq E \cup P, P_{N}(X)=\min _{Z \subseteq E}\left\{\rho_{M}(Z)+\left|X-\bar{Z}^{N}\right|\right\}$

ïi. Para todo $X \subseteq E \cup P, \rho_{N}(X)=\min X \cap E \subseteq \& \subseteq E\left\{\rho_{M}(Z)+\left|X-\bar{Z}^{N}\right|\right\}$

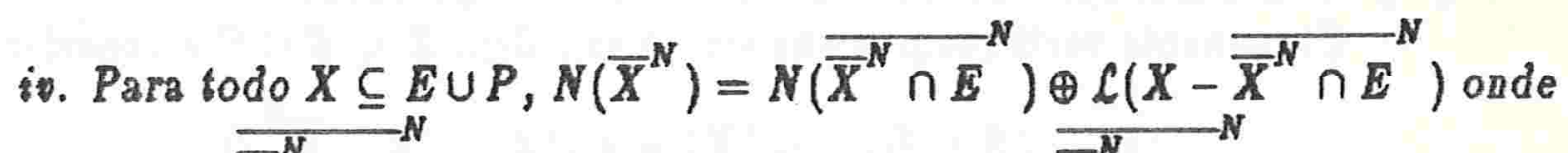
$\mathcal{L}\left(X-\overline{\bar{X}}^{N} \cap \bar{E}^{N}\right)$ é o matróide livre sobre $X-\overline{\bar{X}}^{N} \cap \bar{E}$

Prova: Seguiremos o seguinte roteiro de verificações:

$$
\text { i. } \Leftrightarrow \text { iv. } \Rightarrow \text { ii. } \Rightarrow \text { iii. } \Rightarrow \text { iv. }
$$

A implicação î. $\Rightarrow$ i. é trivial.

Mostremos que i. $\Rightarrow$ iv.. Sejam $X \subseteq E \cup P$ e $Y \subseteq X-\overline{\bar{X}}^{-N} \cap E$. Basta então provarmos que $\overline{\bar{X}}^{N} \cap E \cup Y$ é fechado em $N$. Inicialmente temos que

$$
{\overline{\overline{\bar{X}^{N}}}}^{N} \mathrm{~N} \cup Y \cap E \subseteq \bar{X}^{N} \cap \bar{E} \subseteq{\overline{\bar{X}^{N}} \cap \bar{E}}^{N}
$$


Logo

$$
\begin{aligned}
& {\overline{\overline{\bar{X}}^{N} \cap \bar{E} \cup Y}}^{N}={\overline{\overline{\bar{X}}^{N} \cap E}}^{N} \cup Y \cap \bar{E} \cup\left({\overline{\bar{X}^{N} \cap E}}^{N} \cup Y\right) \\
& \subseteq \overline{\bar{X}}^{N} \cap E N U
\end{aligned}
$$

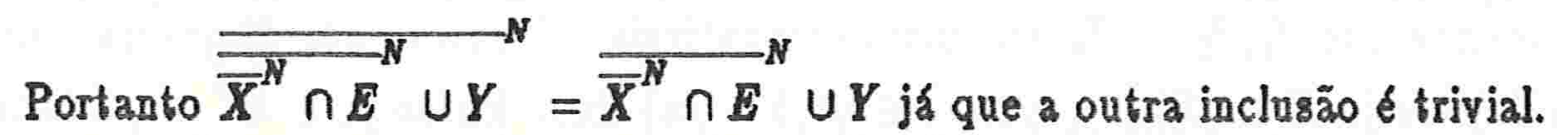

Passemos a verificar que iv. $\Rightarrow$ ii.. Sejam $X \subseteq E \cup P$ e $Z \subseteq E$. Temos então que:

$$
\rho_{N}(X) \leq \rho_{N}\left(\bar{Z}^{N}\right)+\rho_{N}\left(X-\bar{Z}^{N}\right) \leq \rho_{M}(Z)+\left|X-\bar{Z}^{N}\right|
$$

Por iv., lomando $Z=\bar{X}^{N} \cap E$ obtemos

$$
\rho_{N}(X)=\rho_{N}\left(\overline{\boldsymbol{X}}^{N}\right)=\rho_{M}\left(\overline{\boldsymbol{X}}^{N} \cap \bar{E}\right)+\left|X-\overline{\boldsymbol{X}}^{N} \cap E^{N}\right|
$$

Mostremos agora que ii. $\Rightarrow$ iic.. Sejam $X \subseteq E \cup P$ e $Z \subseteq E$ tais que

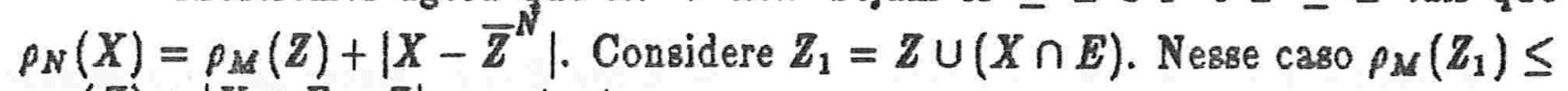
$\rho_{M}(Z)+|X \cap E-Z|$ e portanto

$$
\begin{aligned}
\rho_{M}\left(Z_{1}\right)+\left|X-\bar{Z}_{1}^{N}\right| & \leq \rho_{M}(Z)+\left|X \cap E-\bar{Z}^{N}\right|+\left|X \cap P-\bar{Z}^{N}\right| \\
& =\rho_{M}(\mathbb{Z})+\left|X-\bar{Z}^{N}\right|
\end{aligned}
$$

Logo pela minimalidade de $38 .$, temos na realidade igualdade.

Finalmente verifiquemos que iis. $\Rightarrow i$.. Seja $X \subseteq E \cup P$ e considere

$$
[X]=\left\{Z \in \xi_{M}: \rho_{N}(X)=\rho_{M}(Z)+\left|X-\bar{Z}^{N}\right|\right\}
$$

Com essa definição reescrevemos

$$
\bar{X}^{N}=\left\{x \in E \cup P: \text { existe } Z \in[X] \operatorname{com} x \in \bar{Z}^{N}\right\}
$$

e portanto $\bigcup_{z \in[X]} Z=\bar{X}^{N} \cap E$ eX $-\overline{\bar{X}}^{N} \cap E=\bar{X}^{N}-\bar{X}^{N} \cap E$. Logo

$$
\begin{aligned}
& \rho_{N}(\boldsymbol{X})=\rho_{N}\left(\overline{\boldsymbol{X}}^{N}\right)
\end{aligned}
$$

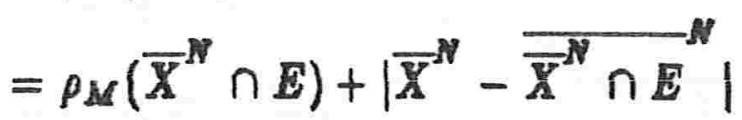

$$
\begin{aligned}
& =\rho_{N}\left({\overline{X^{N}} \cap E}^{N}\right)+\left|\rho_{N}\left(\bar{X}^{N}\right)-{\overline{X^{N}} \cap E}^{N}\right|= \\
& =\rho_{N}\left(\overline{\bar{X}}^{N} \cap E\right)+\left|X-\bar{X}^{N} \cap \mathbb{E}\right|
\end{aligned}
$$


Corolario 2.3. Sejam $A_{1}, A_{2}, \ldots, A_{n}$ subconjuntos de $E$ e $\bar{\beta}_{1}, \xi_{2}, \ldots, \xi_{n}$ os filtros principais associados, isto $e^{\prime} \mathcal{F}_{i}=\left\{X \in \mathcal{F}_{M}: A_{i} \subseteq X\right\}$ para $i=1,2, \ldots, n$. Considere a extensão lisa $N$ de $M$ sobre $E \cup P, P=\left\{p_{1}, p_{2}, \ldots, p_{n}\right\}$, determinada por $\mathcal{F}_{1}, \mathcal{I}_{2}, \ldots, \mathcal{f}_{n}$. Então para todo $X \subseteq E \cup P$

$$
\rho_{N}(X)=\min _{X \cap \bar{E} \subseteq Y \subseteq X}\left\{\rho_{M}\left((Y \cap E) \cup\left\{\bigcup A_{i}: p_{i} \in Y, 1 \leq i \leq n\right\}\right)+|X-Y|\right\}
$$

Prova: Tomemos $X \subseteq E \cup P$. Pelo item iv. do Teorema 2.2. temos

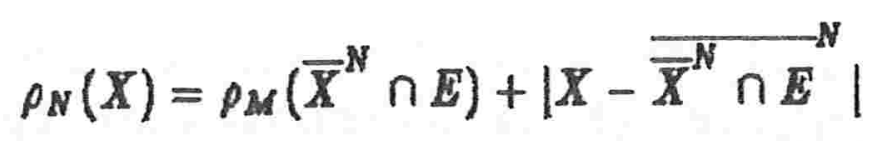

Sejam $Y, Z$ bais que $X \cap E \subseteq Y \subseteq X$ e $Z=(X \cap E) \cup\left\{\cup A_{i}: p_{i} \in Y, 1 \leq i \leq\right.$ $n\}=(Y \cap E) \cup\left\{\cup A_{i}: p_{i} \in Y, 1 \leq i \leq n\right\}$. Temos então que $|X-Y| \geq\left|X-\bar{Z}^{N}\right|$ e do item ii. do Teorema anterior, $\rho_{M}(Z)+\left|X-\bar{Z}^{N}\right| \geq p_{N}(X)$. Combinando esses dois resultados obtemos:

$\rho_{M}\left((Y \cap E) \cup\left\{\bigcup \mathbb{A}_{i}: p_{i} \in Y, 1 \leq i \leq n\right\}\right)+|X-Y|=\rho_{M}(Z)+|X-Y| \geq \rho_{N}(X)$

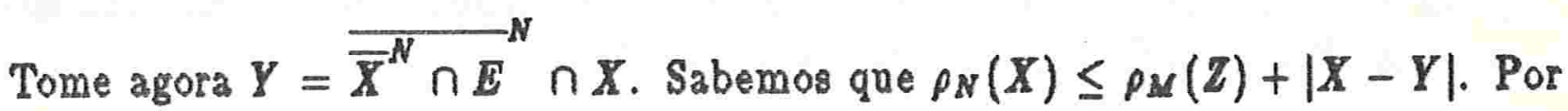
outro lado, de (*)

$$
\rho_{N}(X)=\rho_{M}\left(\bar{X}^{N} \cap E\right)+\left|X-{\overline{\bar{X}^{N}} \cap E}^{N}\right| \geq \rho_{M}(Z)+|X-Y|
$$

pois

(a) $Z \subseteq \bar{X}^{N} \cap E$ :

Seja $x \in \mathbb{Z}$. Se $x \in X \cap \mathbb{E}$ é claro que $x \in \bar{X}^{N} \cap \mathbb{E}$. Tome agora $A_{i} \subseteq Z$. Então $p_{i} \in Y$, donde $p_{i} \in{\overline{\bar{X}^{N} \cap E}}^{N}$ e portanto $A_{i} \subseteq \bar{X}^{N} \cap E$.

(b) $X-\overline{\bar{X}}^{N} \cap \bar{E}=X-Y$ :

A incluaz̃o $\subseteq$ é trivial. Seja $x \in X-Y$. Então $x \in X$ e $x \notin{\overline{\bar{X}^{N}} \cap \bar{E}}^{N} \cap X$,

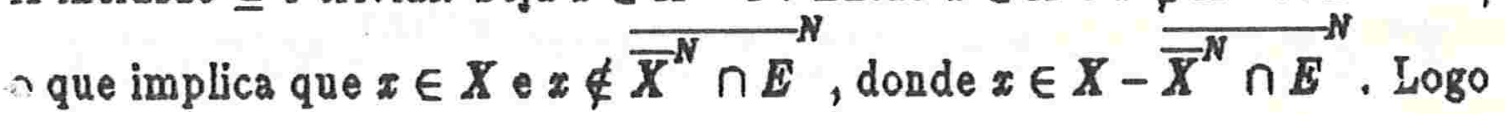
temos a igualdade.

Concluindo, $\rho_{N}(X)=\rho_{M}(Z)+|X-Y|$ para $Y={\overline{\bar{X}^{N} \cap E}}^{N} \cap X$ e $Z=$ $(X \cap E) \cup\left\{\cup \Lambda_{i}: p_{i} \in Y, 1 \leq i \leq n\right\}$. 
Dada uma exteneão $N$ de $M$ e uma sequência ultra-compatível de filtros modulares de $N$, mostraremos uma maneira de recuperar filtros modulares compat́veis de $M$. Estabelecemos também condições suficientes sob as quais esse método fornece filtros modulares ultra-compativeis de $M$.

Proposição 2.4. Seja $N$ uma extensão de $M$ sobre $E \cup P$. Considere a aplicação $\sigma: \bar{F}_{M} \rightarrow \bar{F}_{N}$ tal que $\sigma(X)=\bar{X}^{N}$ para todo $X \in \mathcal{F}_{M}$. Temos então que:

i. $\sigma$ é morísmo forfe de reticulados

ii. Se $\xi_{1}, \xi_{2}, \ldots, \xi_{n}$ são filtros modulares ultra-compativejs de $N$ então $\sigma^{-1}\left(\xi_{1}\right), \sigma^{-1}\left(\xi_{2}\right), \ldots, \sigma^{-1}\left(\xi_{n}\right)$ são filtros modulares compatíveis de $M$ onde

$$
\sigma^{-1}\left(\bar{f}_{i}\right)=\left\{X \in \mathcal{F}_{M}: \bar{X}^{N} \in \bar{f}_{i}\right\} \quad i=1,2, \ldots, n
$$

Prova: O item i. é evidente pois $\overline{X \cup Y}^{N}=\bar{X}^{N} \cup \bar{Y}^{N}$ e $\overline{\bar{X}}^{M^{N}}=\bar{X}^{N}$. Passemos a verificar o item 3 . Mostremos inicialmente que para cada $i=1,2, \ldots, n, \sigma^{-1}\left(\tilde{f}_{i}\right)$ é um filtro modular. Fixemos um valor de $i$. Sejam $X \in \sigma^{-1}\left(\bar{f}_{i}\right)$ e $Y \in \xi_{M}$ tais que $X \subseteq Y$. agora $X, Y \in \sigma^{-1}\left(\bar{\eta}_{i}\right)$ par modular de $M$. Então

$$
\begin{aligned}
\rho_{N}\left(\bar{X}^{N}\right)+\rho_{N}\left(\bar{Y}^{N}\right) & =\rho_{N}(X)+\rho_{N}(Y)=\rho_{M}(X)+\rho_{M}(Y) \\
& =\rho_{M}(X \cup Y)+\rho_{M}(X \cap Y) \\
& \leq \rho_{N}\left(\bar{X}^{N} \cup \bar{Y}^{N}\right)+\rho_{N}\left(\bar{X}^{N} \cap \bar{Y}^{N}\right)
\end{aligned}
$$

donde $\bar{X}^{N}, \bar{Y}^{N}$ par modular em $N$. Logo $\bar{X}^{N} \cap \bar{Y}^{N} \in \bar{f}_{i}$. Mas então $\overline{X \cap Y}^{N}=$ $\bar{X}^{N} \cap \bar{Y}^{N}$ e portanto $X \cap Y \in \sigma^{-1}\left(\boldsymbol{\xi}_{i}\right)$. Falta ainda verificarmos a compatibilidade dos fltros $\sigma^{-1}\left(\tilde{F}_{1}\right), \sigma^{-1}\left(\tilde{F}_{2}\right), \ldots, \sigma^{-1}\left(\tilde{f}_{n}\right)$. Seja $N^{\prime}$ a extensão lisa de $N$ sobre $E \cup P \cup F$, onde $F \cap(E \cup P)=\emptyset$ e $F=\left\{f_{1}, f_{2}, \ldots, f_{n}\right\}$, determinada pelos filtros

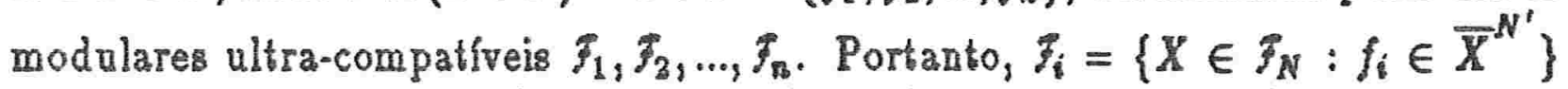
para $i=1,2, \ldots, n$. Seja $M^{\prime}(E \cup F)=N^{\prime}(E \cup F)$ e $M_{i}=M^{\prime}\left(E \cup f_{i}\right), i=1,2, \ldots, n$. E claro que $M^{\prime}(E)=N(E)$ e para cada $i=1,2, \ldots, n$, se $X \in \mathcal{I}_{M}$ e $f_{i} \in \bar{X}^{M_{i}}$ então $\bar{X}^{N} \in \bar{f}_{i}$ e portinto $X \in \sigma^{-1}\left(\bar{f}_{i}\right)$. Logo $M(E) \stackrel{\sigma^{-1}\left(\xi_{i}\right)}{\longrightarrow} M_{i}\left(E \cup f_{i}\right), i=1,2, \ldots, n$.

Na Proposição anterior, $\sigma^{-1}\left(\xi_{1}\right), \sigma^{-1}\left(\xi_{2}\right), \ldots, \sigma^{-1}\left(\xi_{n}\right)$ não são necessariamente ultra-compativeis como mostra o exemplo seguinte: 
Exemplo 2.5. Sejam $E=\{a, b, c, d, e\}, M(E)=\mathcal{L}_{4}(E)$ e $M(E) \stackrel{F}{\longrightarrow} N(E \cup f)$ onde $\xi=\{a b c, c d e, E\}$ fltro modular de $M$. Considere agora $\xi_{1}=\{\text { de, } c f\}_{N}$ e $F_{3}=\{a b, c f\}_{N}$ filtros modulares de $N$. Explicitando,

$$
\begin{aligned}
& \xi_{1}=\{d e, c f, \text { decf, dea, deb, cfab, } E \cup f\} \\
& \xi_{2}=\{a b, c f, a b c f, a b e, a b d, c f d e, E \cup f\}
\end{aligned}
$$

Então $\sigma^{-1}\left(\xi_{1}\right)=[\text { de, } a b c]_{M}$ e $\sigma^{-1}\left(\xi_{2}\right)=\mid a b$, dec $]_{M}$ filtros modulares de $M$, ou

$$
\begin{aligned}
& \sigma^{-1}\left(\tilde{J}_{1}\right)=\{d e, a b c, \text { dec, dea, deb, } E\} \\
& \sigma^{-1}\left(\tilde{J}_{2}\right)=\{a b, \text { dec }, a b c, a b e, a b d, \bar{E}\}
\end{aligned}
$$

$\xi_{1}, \xi_{2}$ s.̃ ultra-compativeis pois $\xi_{1} \cap \xi_{2}=\left\{c f, a b c f_{1}\right.$ decf, $\left.E \cup f\right\}$ e se $X, Y \in \xi_{1} \cap \xi_{2}$ então $X \cap Y \in \xi_{1} \cap \xi_{2}$. Mas $\sigma^{-1}\left(\xi_{1}\right), \sigma^{-1}\left(\xi_{3}\right)$ não são ultra-compativeis pois $a b c$, cde $\in \sigma^{-1}\left(f_{1}\right) \cap \sigma^{-1}\left(f_{2}\right)$, def $(a b c, c d e)=1$ e $a b c \cap c d e=c \notin \sigma^{-1}\left(f_{1}\right) \cap \sigma^{-1}\left(f_{2}\right)$.

A Proposição seguinte nos formece uma condição suficiente para que $\sigma^{-1}\left(\bar{\gamma}_{1}\right), \sigma^{-1}\left(\xi_{2}\right), \ldots, \sigma^{-1}\left(\bar{\gamma}_{n}\right)$ sejam filtros modulares ultra-compatíreis, sob as hjpóteses da Proposição 2.4..

Proposição 2.6. Nas mesmas condições da Proposição 2.4.. Se para cada $i=1,2, \ldots, n, X \in \xi_{i}$ implica que $X \cap E \in \sigma^{-1}\left(\xi_{i}\right)$ então $\sigma^{-1}\left(\xi_{1}\right), \sigma^{-1}\left(\xi_{2}\right), \ldots$, $\sigma^{-1}\left(f_{n}\right)$ são fltros modulares ultra-compativeis. Nesse caso, o seguinte diagrama de extensōes é comutativo;

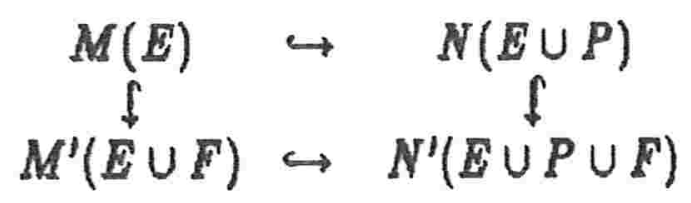

onde $N(E \cup P) \hookrightarrow N^{\prime}(E \cup P \cup F)$ é a extensão lisa determinada por $\xi_{1}, \xi_{2}, \ldots, \xi_{n}$ e $M^{\prime}(E \cup F)=N^{\prime}(E \cup F)$.

Prova: Basta mostrarnos que $M(E) \hookrightarrow M^{\prime}(E \cup F)$ é a extensão lisa de $M$ determinada pelos filtros modulares ultra-compativeis $\sigma^{-1}\left(\xi_{1}\right), \sigma^{-1}\left(\xi_{3}\right), \ldots, \sigma^{-1}\left(\xi_{n}\right)$ o que equivale a mostrar que para todo $X \in E \cup F, \bar{X}^{M^{\prime}} \subseteq{\overline{X^{M^{\prime}}} \cap E}^{M^{\prime}} \cup X$. Seja $X \subseteq E \cup F$ e $x \in \bar{X}^{M^{\prime}}$. Se $x \in \bar{X}^{M^{\prime}} \cap X$ é claro que $x \in \overline{\bar{X}^{M^{\prime}} \cap E} \cup X$. 
Suponha que $x \in \bar{X}^{M^{\prime}}-X$. Então $x \in \bar{X}^{N^{\prime}}-X$. Como $N^{\prime}$ é extensão lisa, $\bar{X}^{N^{\prime}}=\overline{\bar{X}}^{N^{\prime}} \cap(E \cup P) \quad \cup X, \log 0 x \in{\overline{X^{N \prime}}}^{N^{\prime}} \cap(E \cup P)$. Mas $\bar{X}^{N^{\prime}} \cap(E \cup P) \in \bar{\xi}_{i}$ e por hipbtese, $\bar{X}^{N^{\prime}} \cap E \in \sigma^{-1}\left(\mathcal{F}_{i}\right)$. Por outro lado, $\bar{X}^{M^{\prime}} \cap E=\bar{X}^{N^{\prime}} \cap E$ e portanto $x \in \overline{\bar{X}^{M \prime} \cap E} \bar{M}^{\prime}$.

Infeliamente a condição dada na Proposição anterior não é necessária para que os filtros modulares sejam ultra-compatíveis.

Exemplo 2.7. Sejam $E=\{a, b, c\}, M(E)=\mathcal{L}(E)$ e consjdere o seguinte diagrama de extensōes pontuais:

$$
M(E) \stackrel{\delta_{1}}{\longrightarrow} M^{\prime}(E \cup f) \stackrel{\xi_{2}}{\longrightarrow} N(E \cup f \cup p)
$$

onde $\bar{\gamma}_{1}=\{a b, a b c\}$ e $\bar{\gamma}_{2}=\{c f, a b c f\}$. Temos que $\sigma^{-1}\left(\Phi_{2}\right)=\{a b c\}$ é trivialnente um filtro modular ultra-compativel de $M$. Tomando $X=\{c f\} \in \bar{f}_{2}$ temos que $X \cap E=\{c\} \notin \sigma^{-1}\left(\xi_{2}\right)$.
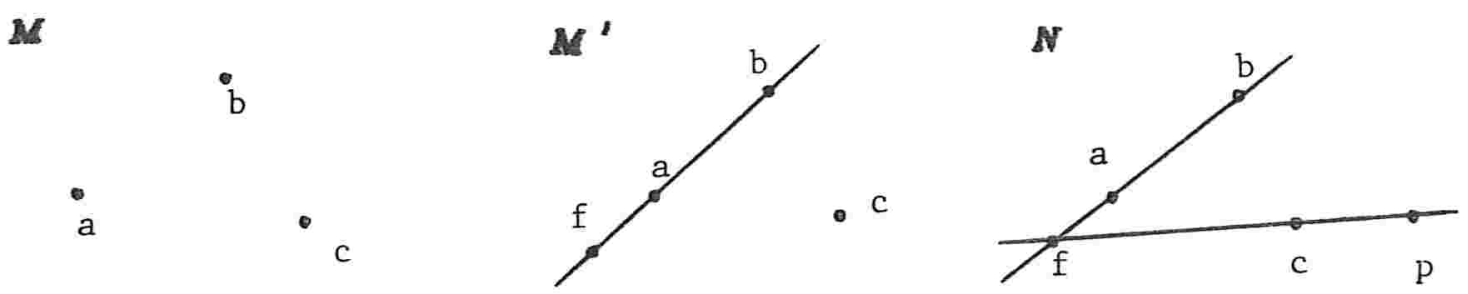

Capitulo II

\section{Extensões Normais e Empilhamentos}

Este é o capítulo mais extenso do srabalho onde examinamos uma classe particular de extensōes de um matróide, as extensones normais, atendo-nos principalmente a suas aplicaçōes na teoria de compatibilidade de extensões.

$\mathrm{Na}$ Seção 1 apresentamos várias caracterizaçōes para a normalidade de conjuntos antes de definirmos extensões normais.

A Seção 2 introdus o conceito de empilhamento de fechados de um matróide. A cada extensão de um matróide podemos associar um empilhamento, mas a cada empilhamento corresponde uma única extensão normal.

Nas Seções 3 e 4 apresentamos três caracterizações dos pares de filtros modulares compatfveis, a primeira devida a Las Vergnas[LV1], a segunda devida a Cheung[Ch] e a última devida a Cordovil[Co2]. 0 principal método aqui abordado consiste em obter um empilhamento de fechados tomando por base um par de filtros modulares e, a partir da extensão sormal associada a esse empilhamento, construir uma 2-extensão relativa a par de filtros mencionado. A caracteriação de Las Vergnas permite mostrar também que o conjunto das 2-extensões de um matroide relativas a um par de filtros modulares fixado formam um semi-reticulado com respeito à ordem fraca. 


\section{NORMALIDADE}

Diremos que $A \subseteq E$ está em posiggẽo normal ou é normal em $M$ se para todo $X \subseteq E-A$ e todo natural $k$ :

$$
\begin{gathered}
\text { se existe } Y_{0} \subseteq A \text { tal que } \rho_{M}\left(Y_{0}\right)=k \text { e } A \subseteq{\overline{X \cup Y_{0}}}^{M} \\
\text { então } A \subseteq \overline{X \cup Y}^{M} \text { para todo } Y \subseteq A \text { tal que } \rho_{M}(Y)=k
\end{gathered}
$$

Observe que todo ponto de $M$ é normal. Infelismente, so investigarmos a existência de exemplos mais interessantes, a definição acima se mostra muito pouco prática. Na tentativa de simplíicá-la apresentamos a seguir, uma caracterisação de normalidade dada pela descrição dos menores do matróide, e uma segunda caracteriação que fornece uma expressão para a função posto.

Proposição 1.1. As seguintes propriedades são equivalentes para $A \subseteq E$ :

i. A é normal em $M$

is. $M(X \cup A) \backslash X=(M(A))_{k}$ para bodo $X \subseteq E-A$ onde $(M(A))_{k}$ indica o truncamento de $M(A)$ ao nivel k (veja pág.6) e

$$
k=\min \left\{j: \text { existe } \mathbb{Z} \subseteq A \text { tal que } p_{M}(\mathbb{Z})=j \text { e } \mathbb{A} \subseteq \overline{X \cup Z}^{\mathbb{Z}}\right\}
$$

63i. $\rho_{M}(X \cup Y)=\min \left(\rho_{M}(X)+\rho_{M}(Y), \rho_{M}(X \cup A)\right)$ para bodo $X \subseteq E-A$ e $Y \subseteq A$

Prova: Seja $X \subseteq E-A$ e

$$
k=\min \left\{j: \text { existe } \mathbb{Z} \subseteq A \operatorname{com} \rho_{M}(Z)=j \text { eA } \subseteq \overline{X \cup Z}^{M}\right\}
$$

Tome $Y_{0} \subseteq A$ tal que $\rho_{M}\left(Y_{0}\right)=k$ e $A \subseteq{\overline{X \cup Y_{0}}}^{M}$.

Mostremos inicialmente que $i \Rightarrow z i$. Podemos supor sem perda de generalidade que $X$ e $Y_{0}$ são independentes em $M$. Seja $Y$ independente em $(M(A))_{k}$. Claramente $Y \subseteq A$ é independente em $M \in|Y| \leq k$. Estendo $Y$ a nma base $Y^{\prime}$ de $(M(\Lambda))_{k}$. Como $\Lambda$ é normal e $\rho_{M}\left(Y^{\prime}\right)=\left|Y^{\prime}\right|=k$ temos que $\Lambda \subseteq \overline{X \cup Y^{\prime}}{ }^{M}$. Suponha que $X \cup Y^{\prime}$ é dependente em $M$. Então existe um circuito $C$ de $M$ tal que $C \subseteq X \cup Y^{\prime}$ e $C-X \neq \emptyset \neq C-Y^{\prime}$. Tome $y \in C-X$. Temos então que

$$
A \subseteq{\overline{X \cup Y^{\prime}}}^{M}={\overline{X \cup\left(Y^{\prime}-y\right)}}^{M}
$$


e

$$
\rho_{M}\left(Y^{\prime}-y\right)=\left|Y^{\prime}-y\right|=k-1
$$

o que contraria a minimalidade de $k$. Portanto $X \cup Y^{\prime}$ é independente em $M$ e como $X \cup Y \subseteq X \cup Y^{\prime}$ temos que $Y$ é independente em $M(X \cup A) / X$, o que mostra que $(M(A))_{k} \subseteq M(X \cup A) / X$. Para verificar a igualdade, mostraremos que $\rho(M(A))_{k}=\rho M(X \cup A) / X$. Seja $Y$ base de $M(X \cup A) / X$. Existe $B$ base de $X \cup A$ tal que $B \cap X$ é base de $X$ e $B \cap A=Y, \log \circ B$ é independente em $X \cup Y$, como $X \cup Y \subseteq X \cup A, B$ é na realidade base de $X \cup Y$. Claramente $\overline{X \cup A}^{M}=\overline{X \cup Y}^{M}$. Temos então que $\mathbb{A} \subseteq \overline{X \cup Y}^{M}$, e pela minimalidade de $k$,

$$
\rho M(X \cup A) / X=|Y| \geq k
$$

Por outro lado, ${\overline{Y_{0}}}^{M / X}=\overline{X \cup Y}^{M}-X=A$ e portanto

$$
k=\left|Y_{0}\right|=\rho_{M} / X\left(Y_{0}\right)=\rho_{M} / X A \geq \rho_{M}(X \cup A) / X A=\rho M(X \cup A) / X
$$

Logo

$$
\rho(M(A))_{k}=k=\rho M(X \cup A) / X
$$

Provemos agora que ii. $\Rightarrow$ iii. Para $Y \subseteq A$ temos que

$$
\rho\left(M \left(A \|_{k}(Y)=\min \left(\rho_{M}(Y), k\right) \text { e } \rho_{M}(X \cup A) / X(Y)=\rho_{M}(X \cup Y)-\rho_{M}(X)\right.\right.
$$

Por

$$
\min \left(\rho_{M}(Y), k\right)=\rho_{M}(X \cup Y)-\rho_{M}(X) \quad(*)
$$

$\operatorname{Mag} k=\rho_{M}(X \cup A) / X(A)=\rho_{M}(X \cup A)-\rho_{M}(X)$. Substituindo em (*) obtemos

$$
\rho_{M}(X \cup Y)=\min \left(\rho_{M}(X)+\rho_{M}(Y), \rho_{M}(X \cup A)\right)
$$

Tinalmente vamos verificar a implicação ioï. $\Rightarrow$ i.. Seja $Y \subseteq A$ tal que $\rho_{M}(Y)=k$. Sabemos que $\rho_{M}(X \cup A)=\rho_{M}\left(X \cup Y_{0}\right)$, logo por ï.

$$
\rho_{M}(X \cup A) \leq \rho_{M}(X)+\rho_{M}\left(Y_{0}\right)=\rho_{M}(X)+\rho_{M}(Y)
$$

donde se conclui que $\rho_{M}(X \cup A)=\rho_{M}(X \cup Y)$. Como $X \cup Y \subseteq X \cup A$ temos que $\overline{X \cup V}^{M}=\overline{X \cup A}^{M}$. Portanto $A \subseteq \overline{X \cup Y}^{M}$, o que nostra que $A$ é normal em M.

Uma extensão $N$ de $M$ sobre $E$ U $P$ é dita normal se $P$ é normal em $N$. 


\section{EMPILHAMENTOS}

Seja $\varepsilon^{(0)}, \varepsilon^{(1)}, \ldots, \mathcal{E}^{(n)}$ uma decomposição do conjunto dos fechados do matroide $M$. Essa decomposição é um empilhamento dos fechados de $M$ se as seguintes propriedades são veríçcadas:

(E1) Para todo par de fechados $X, Y$ de $M$ tais que $X$ cobre $Y$, se $Y \in \mathcal{E}^{(i)}$ então $X \in \mathcal{E}^{(i)} \cup \mathcal{E}^{(i-1)}, \quad i \in\{1,2, \ldots, n\}$

(E2) Para todo par de fechados $X, Y$ de $M$ tais que $X$ e $Y$ cobrem $X \cap Y$, se $X, Y, \overline{X \cup Y}^{M} \in \mathcal{E}^{(i)}$ então $X \cap Y \in \mathcal{E}^{(i)}, \quad i \in\{0,1, \ldots, n\}$

O gras do empilhamento é dado por $\max \left\{i: \varepsilon^{(i)} \neq 0\right\}$. Os filtros modulares podem ser vistos como casos particulares de empilhamentos já que $\varepsilon^{|0|}=\xi$, $\mathcal{E}^{(1)}=\xi_{M}-\mathcal{F}$ é um empilhamento para todo filtro modular $₹$ do matróide $M$. Mais ainda, temos por defnição que $\mathcal{E}^{(0)}$ é um filtro modular qualquer que seja o empilhamento.

Exemplo 2.1. Considere o matróide livre sobre o conjunto $\{a, b, c, d\}$ e o reticulado dos fechados correspondente. Abaixo apresentamos, esquematicamente, vários exemplos de empilhamento:

(1)

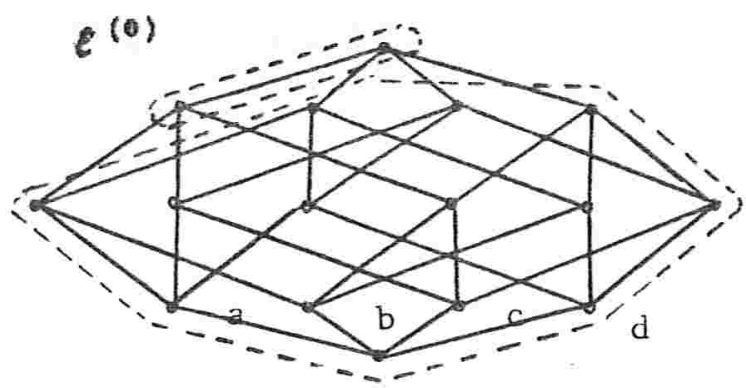

$\varepsilon^{(0)}$
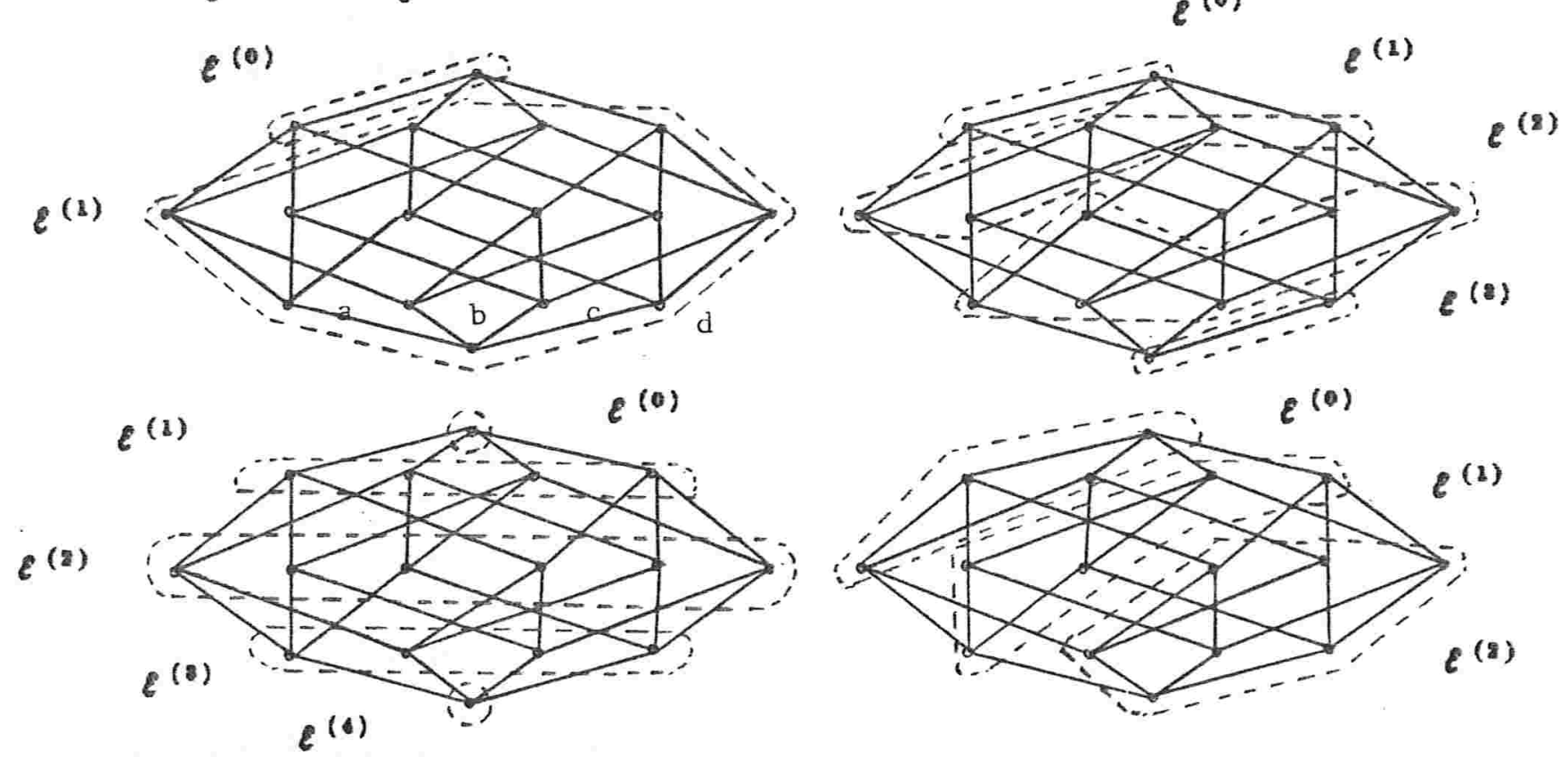

Olhando para o reticulado dos fechados do matróide, observamos que os $\varepsilon^{(3)}$ 's formam faixas dispostas ordenadamente umas sobre as outras. Essa disposição é devida a (E1) e nos sugeriu o termo em pilhamento. Algumas veses vamos nos referir ao empilhamento dos fechados de $M$ simplesmente por empilhamento de $M$. 
Equivalentemente, podemos dizer que uma decomposição do conjunto dos fechados de $M, \varepsilon^{(0)}, \varepsilon^{(1)}, \ldots, \varepsilon^{(n)}$, é um empilhamento se ela tem a propriedade (E1) e a seguinte variante de (E2):

(E2') Para todo par modular $X, Y$ de $M$, se $X, Y, \overline{X \cup Y}^{M} \in \mathcal{E}^{(i)}$ então $X \cap Y \in \mathcal{E}^{(i)}, i \in\{0,1, \ldots, n\}$

Trivialmente (E1) e (E2') implicam (E1) e (E2). Na implicação contrária, (E2') segue de (E1) e (E2) através de uma pequena indução no $p_{M}(X \cup Y)$ $\rho_{M}(X \cap Y)$, observando que:

Proposição 2.2. Seja $L$ um reticulado finito e geométrico, $X, Y \in L$ par modular. Nesse caso existe um sub-reticulado saturado $Q$ de $L$ no intervalo $[X \wedge Y, X \vee Y]$ tal que seus elementos são dois a dois modulares.

Prova: Seja $M$ o matróide associado a $L$ e

$$
\begin{aligned}
& \left\{x_{1}, \ldots, x_{m}\right\} \text { base de } M(X) / X \cap Y \\
& \left\{y_{1}, \ldots, y_{m}\right\} \text { base de } M(Y) / X \cap Y
\end{aligned}
$$

Definimos

$$
W_{i, j}=\overline{(X \cap Y) \cup\left\{x_{1}, \ldots, x_{i}\right\} \cup\left\{y_{1}, \ldots, y_{j}\right\}} i=0,1, \ldots, n \text { e } j=0,1, \ldots, m
$$

Note que $X=W_{s, 0}, Y=W_{0, m}, X \vee Y=\overline{X \cup Y}=W_{n, m}$ e $X \wedge Y=X \cap Y=W_{0.0}$. Esquematicamente:

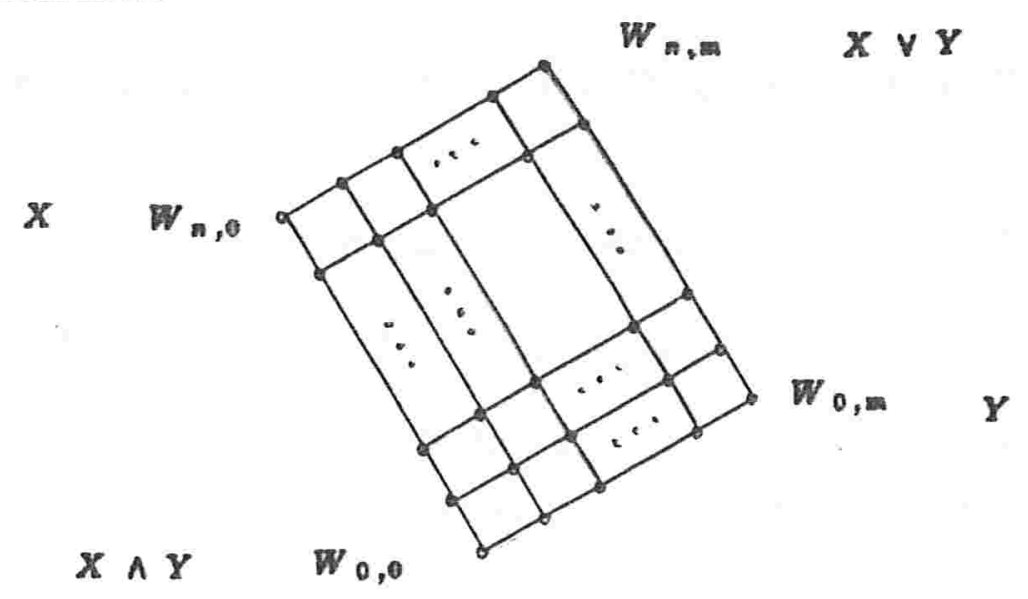

pois temos para todo $i=0,1, \ldots, n$ e $j=0,1, \ldots, m$ que:

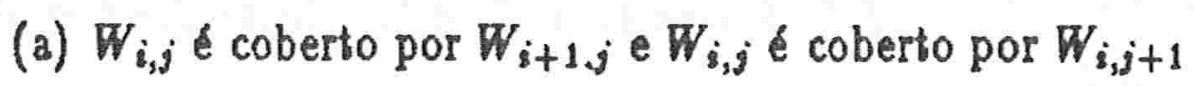

(b) $\rho\left(W_{i, j}\right)=\rho\left(W_{0,0}\right)+i+j$

(c) $W_{i, j} \neq W_{i, l}$ se $i \neq j$ ou $l \neq j$ 
(d) $W_{i, j} \vee W_{k, l}=W_{\max \{i, k\}, \max \{j, l\}} \in W_{i, j} \wedge W_{k, l}=W_{\min \{i, k\}, \min \{j, l\}}$

(e) $W_{i, j}$ e $W_{k . l}$ formam um par modular

Dado um empilhamento $\varepsilon^{(0)}, \varepsilon^{(1)}, \ldots, \varepsilon^{(n)}$ nada impede, a princfpio, a existência de $\mathcal{E}^{(i)}$ 's vasios. A seguir daremos uma condição necessária e suficiente para que $\varepsilon^{(0)}, \varepsilon^{(1)}, \ldots, \varepsilon^{(n)}$ um empilhamento de $M$ de grau n seja uma partição dos fechados de $M$.

Lema 2.8. $\overline{0}^{-M} \in \mathcal{E}^{(n)}$.

Prova: Suponba por absurdo que $\bar{\emptyset}^{M} \in \mathcal{E}^{(k)}$ onde $k<n$. Seja $X \in \mathcal{E}^{(n)}$ e considere a seguinte cadeia de fechados de $M$,

$$
\overline{0}^{\mathbb{M}}=X_{0} \ll X_{1} \ll \ldots \ll X_{l}=X, \quad l \geq 1
$$

onde $X_{i-1} \lessdot X_{i}$ significa que $X_{i}$ cobre $X_{i-1}, i=1,2, \ldots, l$. Pela propriedade (E1) de empilhamento temos que $n \leq k$, uma contradição. Logo $k=n$.

O próximo Lema restringe a disposiçz̃o dos $\mathcal{E}^{(i)}$ 's vasios.

Lema 2.4. Se $\mathcal{E}^{(k)} \neq 0$ para algum $k \in\{0,1, \ldots, n\}$ então $\varepsilon^{(\xi)} \neq \emptyset$ para bodo $i=k, k+1, \ldots, n$.

Prova: Seja $X \in \mathcal{E}^{(k)}$ e considere a seguinte cadeia de fechados de $M$,

$$
\overline{0}^{M}=X_{0} \lessdot X_{1}<\ldots<X_{l}=X
$$

Pela propriedade (E1) de empilhamento e pelo Lema anterior, é fácil ver que para cada $i=k, k+1, \ldots, n$, existe $j_{i} \in\{0,1, \ldots, l\}$ tal que $X_{j_{i}} \in \mathcal{E}^{(i)}$.

Propurição 2.5. $\mathcal{E}^{(0)}, \mathcal{E}^{(1)}, \ldots, \mathcal{E}^{(n)}$ é uma partição do conjunto dos fechados de $M$ se e somente se $E \in \mathcal{E}^{(0)}$.

Prova: Se $E \in \mathcal{E}^{(0)}$ o resultado segue do Lema anterior. Suponha então que $\varepsilon^{(0)}, \varepsilon^{(1)}, \ldots, \varepsilon^{(n)}$ é uma partição do conjunto dos fechados de $M$ e, por absurdo, 
que $E \in \mathcal{E}^{(k)}$ com $k>0$. Analogamente ao que foi feito nos dois últimos Lemas, existe $l$ tal que

$$
\varepsilon^{(0)} \ni X=X_{0} \lessdot X_{1} \lessdot \ldots \lessdot X_{1}=E
$$

e de (E1) conclui-se que $k=0$, uma contradição. Logo $E \in \mathcal{E}^{(0)}$.

Uma das formas de obtermos empilhamentos de um certo matrbide é a partir de suas extensões:

Proposição 2.6. Seja $N$ uma extensão de $M$ sobre $E \cup P$ e $\Lambda \subseteq E \cup P$. Para cada $i=0,1, \ldots, \rho_{N}(A)$ definimos o seguinte conjunto de fechados de $M$

$$
\varepsilon^{(i)}=\left\{X \in F_{M}: p_{N}(X \cup A)-p_{M}(X)=i\right\}
$$

Então $\mathcal{E}^{(0)}, \mathcal{E}^{(1)}, \ldots, \varepsilon^{\left(\operatorname{PN}_{N}(A)\right)}$ é um empilhamento de fechados de grau $\rho_{N}(A)$.

Prova: Verifiquemos que a sequestncia $\varepsilon^{(0)}, \mathcal{E}^{(1)}, \ldots, \varepsilon^{(n)}$, onde $n=p_{N}(\mathbb{A})$, tem as propriedades (E1) e (E2) da definição de empilhamento:

(E1) Sejam $X, Y$ fechados de $M$ tais que $X$ cobre $Y$ e $Y \in \mathcal{E}^{(i)}, i \in\{1,2, \ldots, n\}$. Logo

$$
\rho_{N}(Y \cup A)=\rho_{N}(X \cup A) \text { on } \rho_{N}(X \cup A)-1
$$

e portanto

$$
\begin{aligned}
\rho_{M}(X)=\rho_{M}(Y)+1 & =\rho_{N}(Y \cup A)-i+1 \\
& =\rho_{N}(X \cup A)-i+100 \rho_{N}(X \cup A)-i
\end{aligned}
$$

o que mostra que $X \in \mathcal{E}^{(i)} \cup \mathcal{E}^{(i-1)}$.

(E2) Sejam $X, Y$ fechados de $M$ tais que $X, Y$ cobrem $X \cap Y$ e $X, Y, \overline{X \cup Y}^{M} \in$ $\mathcal{E}^{(i)}, i \in\{0,1, \ldots, n\}$. E claro que $X, Y$ é par modular de $M$, por isso

$$
\begin{array}{r}
p_{N}((X \cap Y) \cup A)-p_{M}(X \cap Y)=p_{M}((X \cap Y) \cup A)-p_{M}(X)- \\
p_{M}(Y)+p_{M}(X \cup Y)
\end{array}
$$

e usando o fato de que $X, Y, \overline{X \cup Y}^{M} \in \mathcal{E}^{(i)}$ temos

$$
\begin{aligned}
& \rho_{N}((X \cap Y) \cup A)-\rho_{M}(X \cap Y)=i+\left(\rho_{N}((X \cup Y) \cup A)+\right. \\
&\left.\rho_{N}((X \cap Y) \cup A)-\rho_{N}(X \cup A)-\rho_{N}(Y \cup A)\right)
\end{aligned}
$$


Para provarmos que $X \cap Y \in \mathcal{E}^{(i)}$ basta mostrarmos que vale

$$
\rho_{N}(X \cup A)+\rho_{N}(Y \cup A)=\rho_{N}((X \cup Y) \cup A)+\rho_{N}((X \cap Y) \cup A)
$$

Da hipotese, existem $x, y \in \bar{B}$ tais que

$$
X=\overline{(X \cap Y) \cup x}^{M}, Y=\overline{(X \cap Y) \cup y}^{M}, \overline{X \cup Y}^{M}=\overline{(X \cap Y) \cup x \cup y}^{M}
$$

E claro que $x, y \notin X \cap Y$ e $y \notin \overline{(X \cap Y) \cup x} \bar{x}^{M}$. Reescrevendo a igualdade a ser provada,

$$
\begin{aligned}
& \rho_{N}((X \cap Y) \cup x \cup A)+\rho_{N}((X \cap Y) \cup y \cup A)= \\
& \rho_{N}((X \cap Y) \cup z \cup y \cup A)+\rho_{N}((X \cap Y) \cup A)
\end{aligned}
$$

Temos três casos para verificar:

Caso 1: $x, y \in{\overline{(X \cap Y) \cup A^{N}}}^{N}$

$A$ igualdade em (*) é facilmente obtida

Caso 2: $x \in{\overline{(X \cap Y) \cup A^{N}}}^{N}$ e $y \notin{\overline{(X \cap Y) \cup A^{N}}}^{N}$ (ou trocando $x$ por $y$ )

(*) decorre do fato de que y $\notin \overline{(X \cap Y) \cup A^{N}}=\overline{(X \cap Y) \cup \pm \cup A}{ }^{N}$

Czso 3: $x, y \notin{\overline{(\mathbb{X} \cap Y) \cup A^{N}}}^{N}$

Suponha por absurdo que $y \in \overline{(X \cap Y) \cup x \cup A}{ }^{N}$. Logo

$$
\begin{aligned}
p_{N}((X \cup Y) \cup A)-\rho_{M}(X \cup Y)= & \rho_{N}((X \cap Y) \cup x \cup y \cup A)- \\
& \rho_{M}(X \cap Y)+z \\
= & \rho_{N}((X \cap Y) \cup x \cup \mathcal{Z})- \\
& \rho_{M}(X \cap Y)+2 \\
= & \rho_{N}(X \cup A)-\rho_{M}(X)+1
\end{aligned}
$$

$O$ que contradiz o fato de $X, \overline{X \cup Y}^{M} \in \mathcal{E}^{(s)}$. Portanto $y$ ${\overline{(X \cap Y) \cup x \cup A^{N}}}^{N}$ donde resulta a igualdade (*).

Resta ainda ierificarmos que $\mathcal{E}^{(0)}, \mathcal{E}^{(1)}, \ldots, \hat{\mathcal{E}}^{(n)}$ é uma decomposição do conjunto dos fechados de $M$. E b́bio que os $\varepsilon^{(i)}$ g são dois a dois disjuntos. Seja $X$ fechado de $M$. Temos então que

$$
\begin{aligned}
\rho_{N}(X \cup A)-\rho_{M}(X) & \leq \rho_{N}(X)+\rho_{N}(A)-\rho_{N}(X \cap A)-\rho_{N}(X) \\
& =\rho_{N}(A)-\rho_{N}(X \cap A) \leq \rho_{N}(A)
\end{aligned}
$$


Logo $X \in \bigcup_{i=0}^{n} \varepsilon^{(i)}$. Falta apenas mostrarmos que $\varepsilon^{(n)} \neq 0$. Mais isso é imediato pois $p_{N}\left(\bar{\phi}^{M} \cup A\right)-p_{M}\left(\bar{\phi}^{M}\right)=n$.

Nas hipoteses da Proposição anterior, o empilhamento

$$
\varepsilon^{(i)}=\left\{X \in \mathcal{J}_{M}: \rho_{N}(X \cup P)-\rho_{M}(X)=i\right\}, \quad i=0,1, \ldots, \rho N
$$

será chamado de empillamento da átenoão $N$ de $M$.

Exemplo 2.T. Seja $M=\mathcal{L}(\{a, b, c\})$ e a seguinte extensz̃o $N$ de $M$ sobre $\{a, b, c, d\}$ :

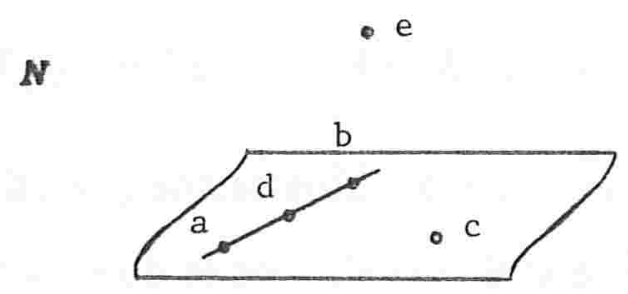

O empilhamento da extensão $N$ de $M$ é dado por:

$$
\varepsilon^{(0)}=\varepsilon^{(1)}=\{a b c, a b\} \quad \varepsilon^{(2)}=\{a c, b c, a, b, c, 0\}
$$

que não é partição de $\xi_{M}$.

A Proposiçz̃o 2.5. nos garante que o empilhamento de uma extensão $N$ de $M$ é uma partição do conjunto dos fechados de $M$ se e somente se $\rho N=\rho M$.

Passemos ao resultado principal desta seção que liga o conceito de empilhamento a conceito de normalidade, fornecendo uma maneira prática de obter extensões normais.

Proposição 2.8. Seja $R$ um matróide sobre $P$ de posto igual a $n$ e $\mathcal{E}^{(0)}, \mathcal{E}^{(1)}, \ldots$, $\mathcal{E}^{(n)}$ um empilhamento de $M$ de grau $n$. Entäo existe uma únjca extensão normal $N$ de $M$ sobre $E \cup P$ tal que $N(P)=R(P)$ e $\varepsilon^{(0)}, \varepsilon^{(1)}, \ldots, \varepsilon^{(n)}$ é o empilhamento da extensão $N$ de $M$. Nesse caso, para todo $X \subseteq E$ e $Y \subseteq P$

$$
\rho_{N}(X \cup Y)=\min \left(\rho_{M}(X)+\rho_{R}(Y), \rho_{M}(X)+i\right)
$$


onde $\bar{X}^{M} \in \mathcal{E}^{(i)}$.

Prova: Verifiquemos que a função $\rho_{N}$ dada pela expressão acima satisfas os seguintes axiomas de posto:

(R1) $\rho_{N}(0)=0$

(R2) Para todo $X \subseteq E, Y \subseteq P$ e $w \in E \cup P$ temos

$$
\rho_{N}(X \cup Y) \leq \rho_{N}(X \cup Y \cup w) \leq \rho_{N}(X \cup Y)+1
$$

(R3) Para todo $X \subseteq E, Y \subseteq P$ e $w, z \in E \cup P$

$$
\begin{gathered}
\text { se } \vartheta_{N}(X \cup Y \cup z)=\emptyset_{N}(X \cup Y \cup w)=\emptyset_{N}(X \cup Y) \\
\text { entaวด } \emptyset_{N}(X \cup Y \cup z \cup w)=\rho_{N}(X \cup Y)
\end{gathered}
$$

A verificação de $(R 1)$ é trivial. Mostremos que (R2) e (R3) valem:

(R1) Seja $X \subseteq E, Y \subseteq P$ e $w \in E \cup P$. Temos dois casos a considerar:

Caso 1: $w \in P$

Usando o lato de que

$$
\rho_{N}(X \cup Y)=\min \left(\rho_{M}(X)+\rho_{R}(Y) \rho_{M}(X)+i\right)
$$

onde $\bar{X}^{M} \in \varepsilon^{(i)} \mathrm{e}$

$$
\rho_{N}(X \cup Y \cup w)=\min \left(\rho_{M}(X)+\rho_{R}(Y \cup w)_{,} \rho_{M}(X)+i\right)
$$

e que $P_{R}$ satigfag (Rz), obtemos

$$
\rho_{N}(X \cup Y) \leq \rho_{N}(X \cup Y \cup w) \leq \rho_{N}(X \cup Y)+1
$$

Caso 2: $w \in E$

Pela definição de $P_{N}$ e pelas propriedades de empillamento

$$
\rho_{N}(X \cup Y)=\operatorname{mix}\left(\rho_{M}(X)+\rho_{R}(Y), \rho_{M}(X)+i\right)
$$

onde $\bar{X}^{M} \in \varepsilon^{(i)} \mathrm{e}$

$$
\rho_{N}(X \cup Y \cup w)=\min \left(\rho_{M}(X \cup w)+\rho_{R}(Y), \rho_{M}(X \cup w)+j\right)
$$


onde $j=i$ ou $i+1$. Se $w \in \bar{X}^{M}$ então $j=i$ e portanto $\rho_{N}(X \cup Y)=\rho_{N}(X \cup Y \cup w)$. Suponha que $w \notin \bar{X}^{M}$. Logo

$$
\rho_{N}(X \cup Y \cup w)=\min \left(\rho_{M}(X)+\rho_{R}(Y)+1, \rho_{M}(X)+j+1\right)
$$

Se $j=i$ então

$$
\rho_{N}(X \cup Y)<\rho_{N}(X \cup Y)+1=\rho_{N}(X \cup Y \cup w)
$$

e se $j=i-1$ entz̃o

$$
\rho_{N}(X \cup Y) \leq \rho_{N}(X \cup Y \cup w) \leq \rho_{N}(X \cup Y)+1
$$

(R3) Seja $X \subseteq E, Y \subseteq P$ e w, $z \in E \cup P$ tais que

$$
\rho_{N}(X \cup Y \cup z)=\rho_{N}(X \cup Y)=\rho_{N}(X \cup Y \cup w)
$$

Da def̂nição,

$$
\rho_{N}(X \cup Y)=\min \left(\rho_{M}(X)+\rho_{R}(Y), \rho_{M}(X)+i\right)
$$

onde $\bar{X}^{M} \in \mathcal{E}^{(i)}$. Se $z=w, z \in \bar{X}^{M} \cup \bar{Y}^{R}$ ou $w \in \bar{X}^{M} \cup \bar{Y}^{R}$ então o axioma é trivialmente verificado. Suponha que $z \neq w$ e $\{z, w\} \cap\left(\bar{X}^{M} \cup \bar{Y}^{R}\right)=\emptyset$. Temos então três casos a considerar:

Caso 1: $z, w \in P$

$$
\begin{aligned}
p_{N}(X \cup Y \cup z)= & \min \left(\rho_{M}(X)+p_{R}(Y)+1, p_{M}(X)+i\right) \\
& =p_{N}(X \cup Y \cup w)
\end{aligned}
$$

Logo

$$
\begin{aligned}
\rho_{N}(X \cup Y) & =\rho_{N}(X \cup Y \cup z)=\rho_{N}(X \cup Y \cup w) \\
& =\rho_{M}(X)+i
\end{aligned}
$$

Mas então

$$
i \leq \rho_{R}(Y)+1 \leq \rho_{R}(Y \cup z \cup w)
$$

e portanto

$$
\begin{aligned}
\rho_{N}(X \cup Y \cup z \cup w) & =\min \left(\rho_{M}(X)+\rho_{R}(Y \cup z \cup w), \rho_{M}(X)+i\right) \\
& =\rho_{M}(X)+i
\end{aligned}
$$


Caso 2: $z \in P$ e $w \in E$

Da detinicăo de $P N$ e de empilhamento ternos:

$$
\begin{gathered}
\rho_{N}(X \cup Y \cup z)=\min \left(\rho_{M}(X)+\rho_{R}(Y)+1, \rho_{M}(X)+i\right) \\
\rho_{N}(X \cup Y \cup w)=\min \left(\rho_{M}(X)+\rho_{R}(Y)+1, \rho_{M}(X)+j+i\right) \\
\text { onde } j=i \text { on } i-1 . \log 0 j=i-1 \mathrm{e} \\
\rho_{N}(X \cup Y \cup z)=\rho_{N}(X \cup Y)=\rho_{N}(X \cup Y \cup w) \\
=\rho_{M}(X)+i
\end{gathered}
$$

Portanto

$$
\begin{aligned}
\rho_{N}(X \cup Y \cup Z \cup w) & =\min \left(\rho_{M}(X)+\rho_{R}(Y)+2, \rho_{M}(X)+j+1\right) \\
& =\min \left(\rho_{M}(X)+\rho_{R}(Y)+\xi, \rho_{M}(X)+i\right) \\
& =\rho_{M}(X)+i
\end{aligned}
$$

Caso 3: $z, w \in E$

Da detiniçä́o de $p_{N}$ e de empilhamento temos:

$$
\begin{aligned}
& \rho_{N}(X \cup Y \cup z)=\min \left(\rho_{M}(X)+\rho_{R}(Y)+1, \rho_{M}(X)+j+1\right) \\
& \text { onde } j=i \text { on } i-1 \text {, e } \\
& \rho_{N}(X \cup Y \cup w)=\min \left(\rho_{M}(X)+\rho_{R}(Y)+1, \rho_{M}(X)+k+1\right) \\
& \text { onde } k=i \text { ou } i-1 . \log 0 j=k=i-1 \mathrm{e} \\
& \begin{aligned}
\rho_{N}(X \cup Y \cup z) & =\rho_{N}(X \cup Y)=\rho_{N}(X \cup Y \cup w) \\
& =\rho_{M}(X)+i
\end{aligned} \\
& \rho_{N}(X \cup Y \cup z \cup w)=\min \left(\rho_{M}(X)+\rho_{R}(Y)+2, \rho_{M}(X)+i\right) \\
& =\rho_{M}(X)+i
\end{aligned}
$$

Logo $\rho_{N}$ é a função posto de um matróide $N$ sobre $E \cup P$. É fácil ver que: 
(a) $N(E)=M(E), N(P)=R(P)$

(b) $\mathcal{E}^{(i)}=\left\{X \in \mathcal{I}_{M}: \rho_{N}(X \cup P)-\rho_{M}(X)=i\right\} \quad i=0,1, \ldots, n$

(c) $P$ é normal em $N$

Portanto, como $N$ está caracteriegdo por $p_{N}, N$ é a única extensão normal de $M$ sobre $E \cup P$ com empilhamento $\varepsilon^{(0)}, \varepsilon^{(1)}, \ldots, \mathcal{E}^{(n)}$ e $N(P)=R(P)$.

Além da existência e unicidade da extensão normal acima, ela é também mínima no seguinte sentido:

Proposição 2.9. Sob as mesmas hipóteses da Proposição anterior. A extensão normal $N$ é minima (em relação à ordem fraca) dentre as extensões $N^{\prime}$ de $M$ sobre $E \cup P$ de empilhamento $\varepsilon^{(0)}, \varepsilon^{(1)}, \ldots, \varepsilon^{(n)}$ e tal que $N^{\prime}(P)=R(P)$.

Prova: Seja $X \subseteq E$ e $Y \subseteq P$. Pela Proposição anterior

$$
\rho_{N}(X \cup Y)=\min \left(\rho_{M}(X)+\rho_{R}(Y), \rho_{M}(X)+i\right)
$$

onde $\bar{X}^{M} \in \mathcal{E}^{(i)}$. Tome $N^{\prime}$ uma extensão de $M$ sobre $E \cup P$ com empilhamento $\varepsilon^{(0)}, \varepsilon^{(1)}, \ldots, \varepsilon^{(n)}$ e tal que $N^{\prime}(P)=R(P)$. Se $\rho_{M}(X)+\rho_{R}(Y) \leq \rho_{M}(X)+i$ então

$$
p_{N}(X \cup Y)=p_{M}(X)+p_{R}(Y)=p_{N^{\prime}}(X)+p_{N \prime}(Y) \geq p_{N}(X \cup Y)
$$

caso contrário

$$
\rho_{N}(X \cup Y)=\rho_{M}(X)+i=\rho_{N^{\prime}}(X \cup P) \geq \rho_{N^{\prime}}(X \cup Y)
$$

Logo $\rho_{N}(X \cup Y) \geq \rho_{N^{\prime}}(X \cup Y)$ para todo $X \subseteq E$ e $Y \subseteq P$, portanto $N \preceq N^{\prime}$.

Como aplicação da teoria desenvolvida neste capítulo daremos uma prova do conhecido resultado devido a Higgs distinta daquela proposta em [Hi].

Proposição 2.10. Sejam $M_{1} \in M_{2}$ matróides sobre $E$ tal que $\xi_{M_{2}} \subseteq \oiint_{M_{1}}$, e seja o conjunto $P=\left\{p_{1}, p_{2}, \ldots, p_{n}\right\}$ tal que $P \cap E=\emptyset$ e $n=\rho M_{1}-\rho M_{2}$. Para cada $i=0,1, \ldots, n$ definimos o seguinte conjunto de fechados de $M_{1}$

$$
\varepsilon^{(i)}=\left\{X \in \mathcal{F}_{M_{1}}: \rho_{M_{1}}(X)-\rho_{M_{2}}(X)=n-i\right\}
$$


Então $\mathcal{E}^{(0)}, \mathcal{E}^{(1)}, \ldots, \mathcal{E}^{(n)}$ é um empilhamento dos fechados de $M_{1}$ de grau n e portanto determina uma única extensão normal $N$ de $M_{1}$ sobre $E \cup P$ tal que $N(P)$ é o matroide livre de grau n. Tem-se ainda que $N \backslash P=M_{1} \in N / P=M_{2}$.

Prova: Verif́quemos que a sequência $\varepsilon^{(0)}, \varepsilon^{(1)}, \ldots, \varepsilon^{(n)}$ tem as propriedades (E1) e (E2) da def̂nição de empilhamento:

(E1) Sejam $X, Y$ fechados de $M_{1}$ tais que $X$ cobre $Y$ e $Y \in \mathcal{E}^{(i)}$. Logo, como $\xi_{M_{2}} \subseteq \xi_{M_{1}}$

$$
\begin{aligned}
P M_{1}(X)-P M_{2}(X) & =P M_{1}(Y)-P M_{3}(Y) O Q P M_{3}(Y)-P M_{2}(Y)+1 \\
& =n-i O n-i+1
\end{aligned}
$$

Portanto $X \in \mathcal{E}^{(i)} \cup \mathcal{E}^{(i-1)}$.

(E2) Sejam $X, Y$ fechados de $M_{1}$ tal que $X$ e $Y$ cobrem $X \cap Y$ e $X, Y, \overline{X \cup Y^{M}} \in$ $\varepsilon^{(i)}$. E claro que $X, Y$ é par modular de $M_{1}$, então

$\rho_{M_{1}}(X \cap Y)-\rho_{M_{2}}(X \cap Y)=\rho_{M_{2}}(X)+\rho_{M_{2}}(Y)-\rho_{M_{1}}(X \cup Y)-\rho_{M_{2}}(X \cap Y)$

e usando o fato de que $X, Y, \overline{X \cup Y}^{M_{2}} \in \xi^{(i)}$ temos

$$
\begin{aligned}
p_{M_{1}}(X \cap Y)-\rho_{M_{2}}(X \cap Y)=n-i & +\rho_{M_{2}}(X)+\rho_{M_{2}}(Y) \\
& -\rho_{M_{2}}(X \cup Y)-\rho_{M_{2}}(X \cap Y)
\end{aligned}
$$

Para provarmos que $X \cap Y \in \mathcal{E}^{(i)}$ basta mostrarmos que vale

$$
\rho_{M_{2}}(X)+\rho_{M_{2}}(Y)=\rho_{M_{3}}(X \cup Y)+\rho_{M_{2}}(X \cap Y)
$$

Da bipótese, existem $x, y \in E$ tais que $X=\overline{(X \cap Y) \cup x}{ }^{M_{3}}, Y=$ $\overline{(X \cap Y) \cup y}{ }^{M_{1}}$ e $\overline{X \cup Y}^{M_{1}}=\overline{(X \cap Y) \cup \approx \cup y}^{M_{1}}$. Reescrevendo a igual. dade a ser provada,

$$
\begin{aligned}
& \rho_{M_{2}}((X \cap Y) \cup x)+\rho_{M_{2}}((X \cap Y) \cup y)= \\
& \rho_{M_{2}}((X \cap Y) \cup x \cup y)+\rho_{M_{3}}(X \cap Y)
\end{aligned}
$$

Temos três casos a verificar:

Caso 1: $x, y \in \overline{X \cap Y}^{M}$,

$A$ igualdade em (*) é facilmente obtida. 
Caso 2: $x \in \overline{X \cap Y}^{M_{3}}$ e y $\notin \overline{X \cap Y}^{M_{2}}$ (ou trocando $x$ por $y$ )

(*) decorre do fato de que $y \notin{\overline{X \cap Y^{M}}}^{M_{2}}=\overline{(X \cap Y) \cup X}^{M_{3}}$.

Caso 3: $x, y \notin{\overline{X \cap Y^{M}}}^{M}$

Suponha por absurdo que $y \in \overline{(X \cap Y) \cup x}^{\mathbb{M}}$. Logo

$$
\begin{aligned}
\rho_{M_{1}}(X \cup Y)-\rho_{M_{3}}(X \cup Y)= & \rho_{M_{1}}((X \cap Y) \cup x \cup y)- \\
& \rho_{M_{2}}((X \cap Y) \cup x \cup y) \\
= & \rho_{M_{1}}(X \cap Y)+2- \\
& \rho_{M_{3}}((X \cap Y) \cup x) \\
= & \rho_{M_{1}}(X)-\rho_{M_{2}}(X)+1
\end{aligned}
$$

0 que contradiz o fato de que $X,{\overline{X \cup Y^{M}}}^{M_{1}} \in \mathcal{E}^{(i)}$. Portanto $y \notin$ ${\overline{(X \cap Y) \cup x^{M}}}^{M_{2}}$ donde resulta a igualdade (*).

Concluf́mos então que $\mathcal{E}^{(0)}, \mathcal{E}^{(1)}, \ldots, \mathcal{E}^{\mid \text {n) }}$ é um empillamento de $M_{1}$ e portanto, pela Proposição 2.8., determina uma única extenzão normal $N$ de $M_{1}$ sobre $E \cup P$ tal que $N(P)$ é o matróide livre de gran n. O posto de $N$ é dado por

$$
\rho_{N}(X \cup Y)=\min \left(\rho_{M_{1}}(X)+|Y|_{,} \rho_{M_{i}}(X)+i\right)
$$

onde $X \subseteq E, Y \subseteq P$ e $\bar{X}^{M_{2}} \in \mathcal{E}^{(i)}$. Temos f́ambém que

$$
\varepsilon^{(i)}=\left\{X \in \mathcal{I}_{M_{1}}: \rho_{N}(X \cup P)-p_{M_{1}}(X)=i\right\} \quad i=0,1, \ldots, n
$$

Logo, se $\bar{X}^{M_{1}} \in \mathcal{E}^{(i)}$ então $\rho_{N}(X \cup P)=\rho_{M_{1}}(X)+i=\rho_{M_{2}}(X)+n$. Reescrevendo a expressão de $\rho_{N}$ obtemos,

$$
\rho_{N}(X \cup Y)=\min \left(\rho_{M_{2}}(X)+|Y|_{\left., \rho_{M_{2}}(X)+n\right)}\right.
$$

onde $X \subseteq E, Y \subseteq P$. Falta verificarmos que $N \backslash P=M_{1}$ e $N / P=M_{2}$. Trivialmente $N \backslash P=M_{1}$ já que $N$ é extensão de $M_{1}$ sobre $\mathbb{E} \cup P$. Para todo $X \subseteq E$ temos

$$
\begin{aligned}
\rho_{N / P}(X) & =\rho_{N}(X \cup P)-\rho_{N}(P)=\rho_{N}(X \cup P)-n \\
& =\left(\rho_{M_{3}}(X)+n\right)-n=\rho_{M_{2}}(X)
\end{aligned}
$$

o que mostra que $N / P=M_{3}$. 
Fechamos esta seção com um pequeno resultado que será útil na última seçẵo deste capítulo.

Proposição 2.11. Seja $N$ uma extensão de $M$ bobre $E \cup P$. Se $N$ é normal então para todo $p \in P$, o fltro modular $\xi_{p}$ que determina a extensão pontual $N(E \cup p)$ de $M$ é dado por

$$
\xi_{p}=\left\{X \in F_{M}: P \subseteq \bar{X}^{N}\right\}
$$

Prova: Seja $p \in P$. Temos que

$$
\xi_{p}=\left\{X \in \xi_{M}: p \in \bar{X}^{N}\right\}
$$

Logo $X \in \bar{\zeta}_{p}$ se e somente se $\rho_{N}(X \cup p)=\rho_{N}(X)=\rho_{M}(X)$. Lembrando o item iii. da Proposição 1.1., temos

$$
\rho_{N}(X \cup p)=\min \left(\rho_{M}(X)+1, \rho_{N}(X \cup P)\right)
$$

e então, $X \in \Phi_{p}$ se e somente se $\rho_{N}(X)=\rho_{N}(X \cup P)$, o que mostra que $X \in \xi_{D}$ se e somente se $P \subseteq \bar{X}^{N}$.

Suspeitamos que no resultado acima a recíproca também vale.

\section{O SDMI.RETICULADO DAS 2.EXTENSÕES}

Apresentaremos aqui o primeiro bloco de uma série de caracterizações dos pares de filtros modulares compatíveis de um matróide. Na primeira caracterigação, devida a Las Vergnas, daremos uma prova levemente modificada, usando o conceito de normalidade, evitando assim uma verificação exaustiva de alguns dos possiveis subcasos r resentes na prova original apresentada em [LV1].

Teorema 3.1. Sejam $\xi_{1}, \xi_{2}$ filtros modulares de $M$ e $\xi_{13}$ um subconjunto de $\xi_{M}-\left(\xi_{1} \cup \xi_{2}\right)$. Então são equivalentes: 
i. Existe $N$ extensão de $M$ sobre $E \cup p_{1} \cup p_{2}$ tal que

$$
\begin{gathered}
\mathcal{J}_{i}=\left\{X \in \bar{F}_{M}: p_{i} \in \bar{X}^{N}\right\} \quad i=1,2 \\
\xi_{13}=\left\{X \in \mathcal{F}_{M}: p_{i} \notin \bar{X}^{N}, i=1_{3}, \quad \text { e } p_{2} \in{\overline{X \cup p_{1}}}^{N}\right\}
\end{gathered}
$$

ii. As seguintes condiçōes são satisfeitas:

P1. Para todo $X \in \mathcal{F}_{M}-\left(\mathcal{F}_{1} \cup \mathcal{F}_{3}\right)$ e $Y \in \mathcal{F}_{1} \cap \mathcal{F}_{2}$, se $Y$ cobre $X$ então $X \in \xi_{12}$

P2. Para todo $X \in \xi_{12}$ e $Y \in \mathcal{f}_{M}$, se $X \subseteq Y$ então $Y \in\left(\xi_{1} \cap \xi_{2}\right) \cup \xi_{12}$

P3. Para todo $X, Y \in \mathcal{J}_{13}$, se $\overline{X \cup Y}^{M} \in \mathcal{I}_{13}$ e $X, Y$ formam um par modular em $M$ entao $X \cap Y \in \Phi_{12}$

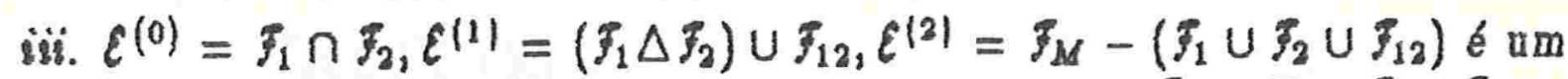
empilhamento dos fechados de $M$ e, para todo $X \in \xi_{12} \in Y \in \xi_{1} \cup \xi_{2}$, se $X \subseteq Y$ então $Y \in \bar{I}_{1} \cap F_{3}$.

Além disso $\bar{\gamma}_{1}, \xi_{2}$ e $\xi_{12}$ verificando P1., P2. e P3. determinam $N$ de modo único. Temos, para todo $X \subseteq E$, que:

$$
\begin{aligned}
\rho_{N}(X) & =\rho_{M}(X) \\
\rho_{N}\left(X \cup p_{i}\right) & = \begin{cases}\rho_{M}(X), & \text { se } \bar{X}^{M} \in \xi_{i} \\
\rho_{M}(X)+1, & \text { caso contrário } i=1,2\end{cases} \\
\rho_{N}\left(X \cup p_{1} \cup p_{2}\right) & = \begin{cases}\rho_{M}(X), & \text { se } \bar{X}^{M} \in \xi_{1} \cap \xi_{2} \\
\rho_{M}(X)+1, & \text { se } \bar{X}^{M} \in\left(\xi_{1} \Delta \xi_{2}\right) \cup \xi_{12} \\
\rho_{M}(X)+z_{1}, & \text { caso contrário }\end{cases}
\end{aligned}
$$

Prova: Mostremos que i. $\Rightarrow$ ï.. Verifiquemos se as condiçōes P1., P2. e P3. estão satisfeitas para $\bar{j}_{1,} \bar{\delta}_{2}$ e $\bar{\xi}_{12}$ dados por $i_{\text {.: }}$

P1. Seja $X \in \xi_{M}-\left(\xi_{1} \cup \xi_{2}\right)$ e $Y \in \xi_{1} \cap \xi_{2}$ tais que $Y$ cobre $X$. Como $X \notin \Phi_{1}$ e $Y \in \bar{f}_{1}$ temos que $\bar{X}^{N} \subset{\overline{X \cup p_{1}}}^{N} \subseteq \bar{Y}^{N}$. Mas $\rho_{N}(Y)=\rho_{N}(X)+1, \log$, $p_{2} \in \bar{Y}^{N}=\overline{X \cup p_{1}}{ }^{N}$ e portanto $X \in \bar{I}_{12}$.

P2. Seja $X \in \mathcal{J}_{13}$ e $Y \in \mathcal{J}_{M}$ tal que $X \subseteq Y$. Então $p_{2} \in{\overline{X \cup p_{1}}}^{N} \subseteq{\overline{Y \cup p_{1}}}^{N}$. Se $p_{1} \in \bar{Y}^{N}$ então $p_{2} \in \bar{Y}^{N}$ e portanto $Y \in F_{1} \cap F_{2}$. Por outro lado, se $p_{1} \notin \bar{Y}^{N}$ então $p_{2} \notin \bar{Y}^{N}$, pois caso conirário $p_{1} \in{\overline{X \cup p_{2}}}^{N} \subseteq \bar{Y}^{N}$ uma contradição. Logo $Y \in \Psi_{12}$. 
P3. Seja $X, Y \in \mathcal{F}_{12}$ par modular de $M$ tal que $\overline{X \cup Y}^{M} \in \xi_{13}$. Afirmo que $X \cap Y \notin \mathcal{J}_{1} \cup \mathcal{J}_{3}$, pois caso contrário $p_{1} \in \overline{X \cap Y}^{N}$ ou $p_{3} \in \overline{X \cap Y}^{N}$, e então $p_{1} \in \bar{X}^{N}$ ou $p_{3} \in \bar{X}^{N}$, donde $X \in \mathcal{F}_{1} \cup \mathcal{F}_{2}$, uma contradição. Do fato de que $\overline{X \cap Y}^{N}=X \cap Y$ e $\rho_{N}\left((X \cap Y) \cup p_{1}\right)=\rho_{M}(X \cap Y)+1$, obtemos

$$
\begin{aligned}
\rho_{N}\left(X \cup p_{1}\right)+\rho_{N}\left(Y \cup \rho_{1}\right) & =\rho_{M}(X)+1+\rho_{M}(Y)+1 \\
& =\rho_{M}(X \cup Y)+\rho_{M}(X \cap Y)+2 \\
& =\rho_{M}\left((X \cup Y) \cup p_{1}\right)+\rho_{M}\left((X \cap Y) \cup p_{1}\right)
\end{aligned}
$$

Não é difícil verificar que para todo par $W, Z \subseteq E$ tal que $\rho_{N}(Z)+\rho_{N}(W)=$ $\rho_{N}(Z \cap W)+\rho_{N}(Z \cup W)$ tem.ge que $\overline{Z \cap W^{N}}=\bar{Z}^{N} \cup \bar{W}^{N}$. Logo

$$
\begin{aligned}
p_{2} \in{\overline{X \cup p_{1}}}^{N} \cap{\overline{Y \cup p_{1}}}^{N} & ={\overline{\left(X \cup p_{1}\right) \cap\left(Y \cup p_{1}\right)}}^{N} \\
& =\overline{(X \cap Y) \cup p_{1}}
\end{aligned}
$$

e portanto $X \cap Y \in I_{12}$.

Passemos a verificar agora que $i i . \Rightarrow$ iii.. Inicialmente temos que $\varepsilon^{(0)}=$ $\xi_{1} \cap \xi_{2}, \varepsilon^{(1)}=\left(\xi_{1} \Delta \xi_{2}\right) \cup \xi_{12}$ e $\varepsilon^{(2)}=\xi_{M}-\left(\xi_{1} \cup \xi_{2} \cup \xi_{12}\right)$ é um empilhamento dos fechados de $M$ pois:

(E1) Sejam $X, Y \in \mathscr{I}_{M}$ tais que $X$ cobre $Y$ e $Y \in \mathcal{E}^{(i)}$.

Se $i=0$ é bbvio que $X \in \mathcal{C}^{(0)}$ ja que $\xi_{1} \cap F_{2}$ é filtro modular.

Se $i=2$, por P1. é claro que $X \in \varepsilon^{(0)}$.

Se $i=1$ então $Y \in \xi_{1} \Delta \xi_{2}$ ou $Y \in \xi_{12}$. No primeiro caso, $X \in \xi_{1} \Delta \xi_{2} \subseteq$ $\varepsilon^{(1)}$ pela propriedade de filtro modular, e no segundo, por $P 2 .$, temos $X \in\left(\xi_{1} \cap \xi_{2}\right) \cup \xi_{12} \subseteq \varepsilon^{(0)} \cup \mathcal{E}^{(1)}$.

Concluindo, $X \in \mathcal{E}^{(i)} \cup \mathcal{E}^{(i-1)}$.

(E2) Sejam $X, Y \in \mathcal{F}_{M}$ tais que $X, Y$ cobrem $X \cap Y$ e $X, Y, \overline{X \cup Y}^{M} \in \varepsilon^{(i)}$. E claro então que $X, Y$ sắ cobertos por $\overline{X \cup Y^{M}}$.

Se $i=2$ por (E1) tem-se que $X \cap Y \in \mathcal{E}^{(2)}$.

Se $i=0$, pela modularidade de $X, Y \in \xi_{1} \cap \xi_{z}$, segue que $X \cap Y \in \mathcal{E}^{(0)}$.

Resta o caso $i=1$. Usando-se propriedade de filtro modular ou P2., é fácil ver que se $X \in \mathcal{F}_{j}$ para $j=1,2$ ou 12 então $\overline{X \cup Y}^{M} \in \xi_{j}$. 0 mesmo ocorre com $Y, \log \circ X, Y, \overline{X \cup Y}^{M} \in f_{j}$ para $j=1,2$ ou 12. Se $j=1$ ou 2 , pela propriedade de filtro modular, $X \cap Y \in \xi_{j}$; nesse caso $X \cap Y \notin F_{1} \cap F_{2}$, pois caso contrário, $X, Y \in \xi_{1}, \xi_{2}$, uma contradição. Se $j=12$, por $\mathrm{P} 3$. conclui-se tamberm que $X \cap Y \in \Psi_{j}$.

Logo, $X \cap Y \in \mathcal{E}^{(i)}$. 
E imediato de P2. que $Y \in T_{1} \subseteq F_{2}$ para todo $X \in F_{12}, Y \in T_{1} \cup F_{3}$ tais que $X \subseteq Y$.

Finalmente, mostremos que $i i_{i} \Rightarrow i$. Tome a extensão normal $N^{\prime}$ de $M$ sobre $E \cup p_{1} \cup p_{2}$ de empilhamento $\mathcal{E}^{(0)}, \varepsilon^{(1)}, \varepsilon^{(3)}$ tal que $N^{\prime}\left(\left\{p_{1}, p_{2}\right\}\right)$ é o matróide livre sobre $\left\{p_{1}, p_{2}\right\}$. Lembrando a Proposição 1.8. temos a seguinte função posto em $N^{\prime}$ :

para todo $X \subseteq E$ e $Y \subseteq\left\{p_{1}, p_{2}\right\}$,

$$
\rho_{N^{\prime}}(X \cup Y)=\min \left(\rho_{M}(X)+|Y|_{2} \rho_{M}(X)+i\right)
$$

onde $\overline{\boldsymbol{X}}^{M} \in \mathcal{E}^{(i)}$. Assim a expressão de $\rho_{N}$ do enunciado pode ser reescrita como: para todo $X \subseteq E$,

$$
\begin{aligned}
\rho_{N}(X) & =\rho_{M}(X) \\
\rho_{N}\left(X \cup p_{i}\right) & = \begin{cases}\rho_{M}(X), & \text { se } \bar{X}^{M} \in \mathcal{F}_{i} \\
\rho_{M}(X), & \text { caso contrário } \quad i=1,2\end{cases} \\
\rho_{N}\left(X \cup p_{1} \cup p_{2}\right) & =\rho_{N}\left(X \cup p_{1} \cup p_{2}\right)
\end{aligned}
$$

Verifiquemos que $\rho_{N}$ satisfaz os seguintes axiomas de posto:

(R1) $\rho_{N}(\theta)=0$

(R2) Para todo $Z \subseteq E \cup p_{1} \cup p_{2}$ e $x \in E \cup p_{1} \cup p_{2}$

$$
\rho_{N}(Z) \leq \rho_{N}(Z \cup x) \leq \rho_{N}(Z)+1
$$

(R3) Para todo $Z \subseteq E \cup p_{1} \cup p_{2} \in x_{3} y \in E \cup p_{1} \cup p_{2}$,

$$
\begin{gathered}
\text { se } \rho_{N}(Z \cup x)=\rho_{N}(Z \cup y)=\rho_{N}(Z) \\
\text { entวัด } \rho_{N}(Z \cup ⿰ \cup y)=\rho_{N}(Z)
\end{gathered}
$$

Observe inicialmente que para $i=1,2, \rho_{N}$ restrito a $E \cup p_{i}$ é a fonção posto da extensão pontual de $M$ pelo filtro modular $\xi_{i}$. Logo é suficiente verificar (R2) quando $\left\{p_{1}, p_{2}\right\} \subseteq Z \cup x$ e (R3) quando $\left\{p_{1}, p_{2}\right\} \subseteq Z \cup z \cup y$. Podemos supor também que $\left\{p_{1}, p_{2}\right\} \& Z$ pois $p_{N}$ restrito a conjuntos que contêm $\left\{p_{1}, p_{2}\right\}$ coincide com a funç⿸厃㔾 posto $P_{N}$.

(R2) $Z=X \cup p_{1}, X \subseteq E$ e $=p_{2}$ é o único caso que necessita verificação. Se $\bar{X}^{M} \in \mathcal{f}_{1}$ entäo $\bar{X}^{M} \in \mathcal{E}^{(0)} \cup \mathcal{E}^{(1)}, \log \circ$

$$
\rho_{N}(Z)=\rho_{M}(X) \leq \rho_{N}(Z \cup x) \leq \rho_{M}(X)+1=\rho_{N}(Z)+1
$$


Por outro lado, se $\bar{X}^{M} \notin \mathcal{F}_{1}$ então $\bar{X}^{M} \in \mathcal{E}^{(1)} \cup \mathcal{E}^{(2)}$, logo

$$
\rho_{N}(Z)=\rho_{M}(X)+1 \leq \rho_{N}(Z \cup Z) \leq \rho_{M}(X)+2=\rho_{N}(Z)+1
$$

(R3) Seja $Z \subseteq E \cup p_{1} \cup p_{2}$ e $x, y \in E \cup p_{1} \cup p_{2}$ tais que $\left\{p_{1}, p_{2}\right\} \subseteq Z \cup z \cup y$, $\left\{p_{1}, p_{2}\right\} \nsubseteq Z$ e $\rho_{N}(Z \cup x)=\rho_{N}(Z \cup y)=\rho_{N}(Z)$. Temos então duas possibilidades:

Caso 1: $Z \subseteq E_{1} z=p_{1}$ e $y=p_{2}$

Então para cada $i=1,2$ temos

$$
P_{N}\left(Z \cup p_{i}\right)=\rho_{N}(Z)=P_{M}(Z)
$$

Logo $\bar{Z}^{M} \in \mathcal{F}_{1} \cap \mathcal{F}_{2}$ e portanto

$$
\begin{aligned}
\rho_{N}(Z \cup \mathcal{Z} \cup y) & =\rho_{N^{\prime}}\left(Z \cup p_{1} \cup p_{2}\right) \\
& =\rho_{M}(Z)=\rho_{N}(Z)
\end{aligned}
$$

Caso 2: $z=X \cup p_{1}, X \subseteq E, x \in E, y=p_{2}$ (ou trocando $p_{1}$ com $p_{2}$ ) Se $x \in \bar{X}^{M}$ então

$$
\rho_{N}(Z \cup x \cup y)=p_{N}(Z \cup y)=\rho_{N}(Z)
$$

Suponhamos que $z \notin \bar{X}^{M}$, nesse caso, $\rho_{N}(Z \cup x)=\rho_{N}(Z)$ e $\rho_{M}(X \cup X)=\rho_{M}(X)+1$ implicam que $\bar{X}^{M} \xi_{1}$, donde $\rho_{N}(Z)=$ $\rho_{M}(X)+1$ e $\overline{X \cup X} \in \mathscr{f}_{1}$. Como $p_{N}(\mathbb{Z})=\rho_{N}\left(Z \cup p_{3}\right)$ então $\bar{X}^{M} \in\left(\tilde{f}_{1} \Delta \tilde{F}_{2}\right) \cup \tilde{f}_{13}=\varepsilon^{(1)}$. Combinando com o fato de que $\bar{X}^{M} \notin \bar{f}_{1}$ tem-se que $\bar{X}^{M} \in\left(\xi_{3}-\xi_{1}\right) \cup \xi_{12}$. Se $\bar{X}^{M} \in \bar{z}_{2}-\xi_{1} \varepsilon$ claro que $\overline{X \cup x} \in \mathcal{I}_{1} \cup \mathcal{F}_{2}$. Por outro lado, se $\bar{X}^{M} \in \mathcal{F}_{12}$ usando a propriedade (E1) de empilbamento temos que $\overline{X \cup{ }^{M}} \in \mathcal{f}_{1} \cup f_{3}$, logo por P2., $\overline{X \cup X}^{M} \in \tilde{F}_{1} \cap \bar{F}_{2}=\varepsilon^{(0)}$. Portanto

$$
\begin{aligned}
\rho_{N}(Z \cup x \cup y) & =\rho_{N}\left(X \cup p_{1} \cup p_{2} \cup x\right) \\
& =\rho_{M}(X \cup x)=\rho_{N}(Z)
\end{aligned}
$$

Seja $N$ o único matróide sobre $E \cup p_{1} \cup p_{2}$ determinado por $\rho_{N}$. É fácil ver pela definição de $\rho_{N}$ que:

$$
N(E)=M(E)
$$




$$
\begin{gathered}
\xi_{i}=\left\{X \in \mathcal{F}_{M}: p_{i} \in \bar{X}^{N}\right\} \quad i=\mathbb{1}, 2 \\
\xi_{12}=\left\{X \in \xi_{M}: p_{i} \notin \bar{X}^{N}, i=1,2 \text { e } p_{3} \in{\overline{X \cup p_{1}}}^{N}\right\}
\end{gathered}
$$

Até o final da seção, $\xi_{1}, \xi_{2}$ são dois filtros modulares de $M$ e $\xi_{12} \subseteq \xi_{M}-$ $\left(\xi_{1} \cup \xi_{2}\right)$. A propriedade $\mathrm{P} 2$. é equivalente à conjunção das seguintes propriedades:

P2.1. Para todo $X \in f_{12}$ e $Y \in \xi_{M}-\left(f_{1} \cup \xi_{3}\right)$, se $X \subseteq Y$ então $Y \in f_{12}$

P2.2. Para todo $X \in \xi_{12}$ e $Y \in \xi_{1} \cup \xi_{2}$, se $X \subseteq Y$ então $Y \in \xi_{1} \cap \xi_{2}$

Note que se $\xi_{12}=\xi_{M}-\left(\xi_{1} \cup \xi_{2}\right)$ então trivialmente P1., P2.1. e P3. se verificam. Também, se duas partes de $\xi_{M}-\left(\xi_{1} \cup \xi_{2}\right)$ verificam P1., P2.1. e P3, então a intersecção dessas partes também verifica. Denotemos por $\left[\boldsymbol{f}_{1}, \boldsymbol{f}_{2}\right]$ a intersecção de todas as partes de $\xi_{M}-\left(\xi_{1} \cup \xi_{2}\right)$ que verificam P1., P2.1. e P3..

O Teorema anterior estabelece uma bijeção entre $\Gamma\left(\xi_{1}, f_{2}\right)$ e $\Phi\left(\xi_{1}, f_{2}\right)$, onde $\Gamma\left(\xi_{1}, \xi_{2}\right)$ denota o conjunto de todas as partes de $\xi_{M}-\left(\xi_{1} \cup \xi_{2}\right)$ que veríicam $P 1, P 2$. e $P 3$, e $\Phi\left(I_{1}, \Phi_{2}\right)$ denota o conjunto de todas as 2-extensöes $N$ de $M$ sobre $E \cup p_{1} \cup p_{2}$.

Proposição 3.2. Sejam $\bar{\xi}_{1}$ e $\bar{\xi}_{2}$ dois filtros modulares de $M$. Então $\xi_{1}, \xi_{2}$ sz̃o compatíveis se e somente se para todo $X \in\left[\tilde{F}_{1}, F_{3}\right]$ e para todo $Y \in \mathcal{F}_{1} \Delta \xi_{2}$ temos que $X \nsubseteq Y$.

Prova: Suponha que $\bar{\xi}_{1}$ e $\bar{\zeta}_{2}$ são compativeis e considere uma extensão $N$ de $M$ sobre $E \cup p_{1} \cup p_{2}$ tal que

$$
\bar{f}_{i}=\left\{X \in \bar{F}_{M}: p_{i} \in \bar{X}^{N}\right\} \quad i=1,2
$$

Seja

$$
\xi_{12}=\left\{X \in \xi_{M}: p_{i} \notin \bar{X}^{N}, i=1,2 \text { e } p_{2} \in{\overline{X \cup p_{1}}}^{N}\right\}
$$

Pelo Teorema anterior temos que $\xi_{1}, \xi_{2}$ e $\tau_{12}$ verificam P1. P2. e P3.. Logo $\left[\xi_{1}, \xi_{3}\right] \subseteq \xi_{13}$ e, para todo $X \in\left[\xi_{1}, \xi_{2}\right], Y \in \xi_{M}$, se $X \subseteq Z$, P2. implica que $Y \subseteq\left(\bar{\xi}_{1} \cap \bar{\xi}_{2}\right) \cup \bar{\xi}_{12}$.

Suponha agora que para todo $X \in\left[\tilde{f}_{1}, \boldsymbol{F}_{2}\right]$ e para todo $Y \in \boldsymbol{F}_{1} \Delta \boldsymbol{F}_{2}$ temos que $X \nsubseteq Y$. Mostremos que $\xi_{12}=\left[\xi_{1}, \xi_{2}\right]$ satisfaz P2.2. pois nesse caso P1., P2. e P3, estão verificadas e portanto, pelo Teorema anterior, $\xi_{1}$ e $\xi_{2}$ são compatíveis. 
Mas P2.2. é trivialmente satisfeita já que se $X \in\left[\xi_{1}, \xi_{2}\right], Y \in \xi_{1} \cup \xi_{2}$ e $X \subseteq Y$, é claro que $Y \in \mathcal{F}_{1} \cap \xi_{2}$.

A áltima Proposição nos diz que se $\xi_{1}, \xi_{2}$ são compat̂́eis então $\left[\xi_{1}, \xi_{2}\right]$ satisfar P2.2. e portanto é mínimo em $\Gamma\left(\boldsymbol{F}_{1}, \boldsymbol{J}_{2}\right)$ (com respeito à inclusão de conjuntos). A partir desse fato conseguimos obter informaçōes adicionais relativas à bijeção existente entre $\Gamma\left(\xi_{1}, \xi_{2}\right)$ e $\Phi\left(\xi_{1}, \xi_{2}\right)$.

Proposição s.శ. A bijeção entre $\Gamma\left(f_{1}, f_{2}\right)$ e $\Phi\left(f_{1}, f_{2}\right)$ dada pelo Teorema 3.1. é um isomorfismo entre os conjuntos ordenados $(\Gamma, \subseteq)$ e $(\Phi, \preceq)$. Em particular $(\Phi, \preceq)$ é um $\wedge$-semi-reticulado onde o elemento de $\Phi$ correspondente a $\left[\tilde{f}_{1}, \hat{f}_{3}\right] \in \Gamma$ é mínimo em $\Phi$ em relação à ordem fraca $\preceq$.

Prova: Sejam $\pi_{12}, z_{12}^{\prime} \in \Gamma$ e $N, N^{\prime} \in \Phi$ as extensones correspondentes dadas pelo Teorema 3.1.. Para provarmos que a bijeção dada pelo Teorema 3.1. é um isomorfismo entre $(\Gamma, \subseteq)$ e $(\Phi, \preceq)$, basta mostrarmos que

\section{$\xi_{12} \subseteq \xi_{12}^{\prime}$ se e somente se $N \preceq N^{\prime}$}

Suponhamog inicialmente que $N \preceq N^{\prime}$, igto é, $\rho_{N}(Y) \geq \rho_{N}(Y)$ para todo $Y \in E \cup p_{1} \cup p_{2}$. Seja $X \in \bar{f}_{12}$. Então $p_{i} \notin \bar{X}^{N}$ para $i=1,2$ e $p_{2} \in{\overline{X \cup p_{1}}}^{N}$, logo

$$
\rho_{N^{\prime}}\left(X \cup p_{1} \cup p_{2}\right) \leq \rho_{N}\left(X \cup p_{1} \cup p_{2}\right)=\rho_{N}\left(X \cup p_{1}\right)
$$

Mas como $N\left(E \cup p_{1}\right)$ e $N^{\prime}\left(E \cup p_{1}\right)$ coincidem com a extensão pontual de $M$ sobre $E \cup p_{1}$ pelo filtro modular $\xi_{1}$ então $\rho_{N^{\prime}}\left(X \cup p_{1} \cup p_{2}\right) \leq \rho_{N^{\prime}}\left(X \cup p_{1}\right)$. Logo

$$
\rho_{N^{\prime}}\left(X \cup \eta_{1} \cup p_{2}\right)=\rho_{N^{\prime}}\left(X \cup p_{1}\right)
$$

donde $p_{2} \in{\overline{X \cup p_{1}}}^{N^{\prime}}$, e portanto $X \in \xi_{12}^{\prime}$.

Suponhamos por outro lado que $\xi_{12} \subseteq \xi_{12}^{\prime}$ e mostremos que $N \preceq N^{\prime}$. E fácil ver pela expressão de $\rho_{N}$ e $\rho_{N}$ dadas pelo Teorema 3.1. que para todo $X \subseteq E$ :

$$
\begin{aligned}
\rho_{N}(X) & =\rho_{N}(X) \\
\rho_{N}\left(X \cup p_{i}\right) & =\rho_{N^{\prime}}\left(X \cup p_{i}\right) \quad i=1,2 \\
\rho_{N}\left(X \cup p_{1} \cup p_{2}\right) & = \begin{cases}\rho_{N^{\prime}}\left(X \cup p_{1} \cup p_{3}\right), & \text { se } \bar{X}^{M} \in\left(\xi_{M}-\tilde{\xi}_{13}^{\prime}\right) \cup \xi_{13} \\
\rho_{N}\left(X \cup p_{1} \cup p_{3}\right)+1, & \text { csoo contrário }\end{cases}
\end{aligned}
$$


Logo $\rho_{N}(Y) \geq \rho_{N}(Y)$ para todo $Y \subseteq E \cup p_{1} \cup p_{2}$. As demais afirmações feitas no enunciado desta Proposição decorren então do fato de que $(\Gamma, \subseteq)$ é um $\wedge$ semi-reticulado, da Proposiçz̃o 3.2. e de que $\left[\boldsymbol{f}_{1}, \boldsymbol{\xi}_{2}\right]$ é mínimo em $\Gamma$ em relação à inclusão de conjuntos.

Podemos finalmente enunciar e provar a caracterisação devida a Cheung mencionada no ińficio do capítulo.

Proposição 8.1. Sejam $\xi_{1}, \xi_{2}$ filtros modulares de $M$ tais que

$$
M(E) \stackrel{\xi_{1}}{\longrightarrow} N_{1}\left(E \cup p_{1}\right) \text { e } M(E) \stackrel{\xi_{3}}{\longrightarrow} N_{2}\left(E \cup p_{3}\right)
$$

Entวิ são equivalentes:

i. $\xi_{1}$ e $\xi_{2}$ são compatíneis

ii. O seguinte diagrama é comutativo

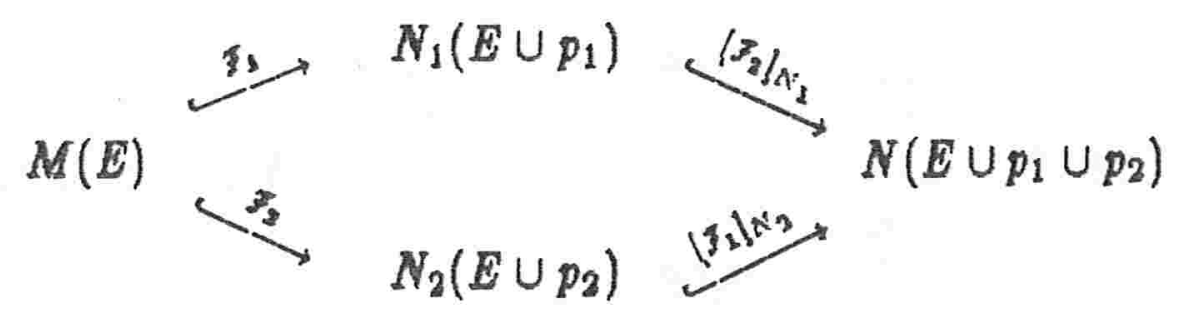

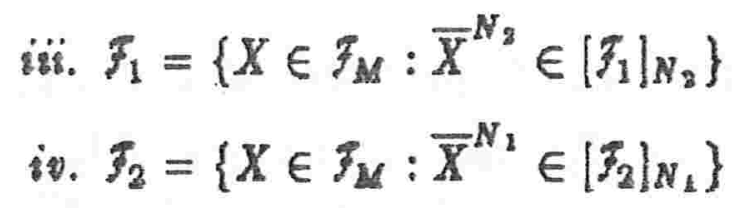

Prova: Seguiremos o seguinte roteiro de verificações:

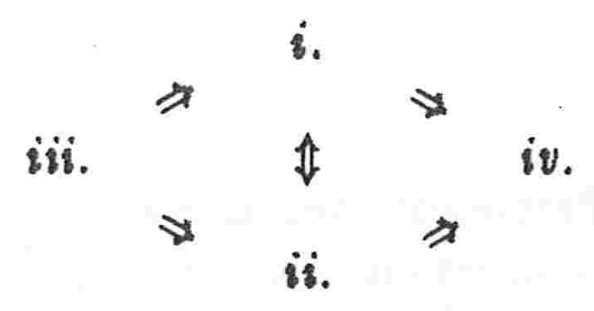


A implicação $\ddot{3 i} \Rightarrow i$. É imediata. Mostremos então que $i . \Rightarrow \ddot{i i .} \Rightarrow$ iii. $\Rightarrow i$. já que $i i . \Rightarrow i v, \Rightarrow i$. seguem por simetria.

E fásil ver que $i i . \Rightarrow$ sii. Trivialmente $\xi_{1} \subseteq\left\{X \in \xi_{M}: \bar{X}^{N_{2}} \in\left[\xi_{1}\right]_{N_{2}}\right\}$. A incluaz̃o contrária segue do fato de que se $X \in I_{M}$ e $\bar{X}^{N_{2}} \in\left[\tilde{f}_{1}\right]_{N_{3}}$ então $p_{1} \in \bar{X}^{N}$; mas $\bar{X}^{N_{1}}=\bar{X}^{N}-p_{2}, \log 0 p_{1} \in \bar{X}^{N_{1}}$, donde $X \in z_{1}$.

Para verificarmos que ïis. $\Rightarrow i_{\text {. }}$ considere a extensão $N$ de $M$ sobre $E \cup p_{1} \cup p_{2}$ dada pelo seguinte diagrama:

$$
M(E) \stackrel{F_{2}}{\longrightarrow} N_{2}\left(E \cup p_{2}\right) \stackrel{\left|F_{1}\right|_{N_{2}}}{\longrightarrow} N\left(E \cup p_{1} \cup p_{2}\right)
$$

Basta mostrarmos que $N_{1}\left(E \cup p_{1}\right)=N\left(E \cup p_{1}\right)$. Seja $X$ fechado de $M$, então

$$
X \in I_{1} \Leftrightarrow \bar{X}^{N_{2}} \in\left[p_{1}\right]_{N_{2}} \Leftrightarrow p_{1} \in \bar{X}^{N} \Leftrightarrow p_{1} \in \bar{X}^{N\left(Z \cup p_{1}\right)}
$$

Reşa a implicação $i \Rightarrow$ is. para ser verificada. Suponha que $f_{1}$ e $f_{2}$ são compativeis. Seguindo a notação da Proposição 3.3., seja $N^{t}$ o elemento mínimo de $\Phi\left(f_{1}, f_{2}\right)$ em relação à ordem fraca $\preceq$. Então exiatem $f_{1}^{\prime}, f_{2}^{\prime}$ filtros modulares de $N_{3}, N_{1}$ respectivamente, tais que o diagrama abaixo é comutativo

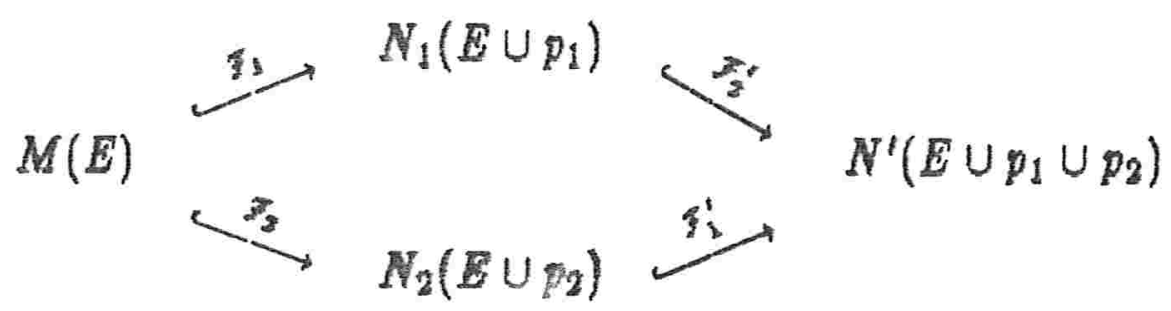

Pela minimalidade de $N^{\prime}, \rho_{N}(Z) \geq \rho_{N}(Z)$ para todo $Z \subseteq E \cup p_{1} \cup p_{2}$. Se $X \in F_{2}$ então $p_{2} \in \bar{X}^{N^{\prime}}$, donde $\bar{X}^{N^{\prime}} \in F_{2}^{\prime}$. Isso mostra que $\left|\xi_{2}\right|_{N_{1}} \subseteq \xi_{3}$. Examinando os diagramas de extensões pontuais que dão origem a $N$ e $N^{\prime}$ é fácil ver então que $\rho_{N}(Z) \geq \rho_{N^{\prime}}(Z)$ para todo $Z \subseteq E \cup p_{1} \cup p_{2}$. Concluímos que $N=N^{\prime}$ e portanto $\left[\xi_{2}\right]_{N_{2}}=\xi_{3}^{\prime}$ e $\left[\xi_{1}\right]_{N_{2}}=\xi_{1}^{\prime}$.

Na prova dessa Proposição chegamos à conclusão de que a extensão $N$ do item $\ddot{3}$. é exatamente o elemento mínimo de $\Gamma\left(\tilde{f}_{1}, \xi_{2}\right)$ em relação Esse resultado será útil na próxima seção quando formos provar o Teorema 4.3.. 


\section{2-COMPATIBILIDADE}

Seja $F$ um flltro modular de $M$ e considere o seguinte diagrama de extensões pontuais:

$$
M(E) \stackrel{\xi}{\longrightarrow} M_{1}\left(E \cup p_{1}\right) \stackrel{[\xi]_{M_{1}}}{\longrightarrow} M_{2}\left(E \cup p_{1} \cup p_{2}\right)
$$

Diremos que $\xi$ um filtro modular 2 -compativel de $M$ se no diagrama acima, $\rho_{M_{2}}\left(\left\{p_{1}, p_{2}\right\}\right)=2.0$ empillamento da extensão $M_{2}$ de $M$

$$
\varepsilon^{(i)}=\left\{X \in \mathscr{F}_{M}: \rho_{M_{2}}\left(X \cup p_{1} \cup p_{2}\right)-\rho_{M}(X)=i\right\} \quad i=0,1,2
$$

será chamado de empilhamento determinado pelo filtro modular 2-compatínel 8 . E fácil ver que $\varepsilon^{(0)}=\xi$ e $\overline{0}^{M} \in \mathcal{E}^{(2)}, \log 0$, lembrando a Proposição 2.5., 0 empilhamento particiona os fechados de $M$.

Nos próximos dois resultados relacionamos a 2-compatibilidade à normalidade.

Lema 4.1. Considere o seguinte diagrama de extensões pontuais

$$
M(E) \stackrel{\xi_{1}}{\longrightarrow} M_{1}\left(E \cup p_{1}\right) \stackrel{\xi_{2}}{\longrightarrow} M_{3}\left(E \cup p_{1} \cup p_{3}\right)
$$

onde $\xi_{1}$ e $\xi_{2}$ são fitros modulares de $M$ e $M_{1}$ respectivamente. Então para todo $X \subseteq E$ temos:

$$
\begin{aligned}
& \rho_{M_{2}}(X)=\rho_{M}(X) \\
& \rho_{M_{2}}\left(X \cup p_{1}\right)= \begin{cases}\rho_{M}(X), & \text { se } \overline{X^{M}} \in I_{1} \\
\rho_{M}(X)+1, & \text { caso contrário }\end{cases} \\
& \rho_{M_{3}}\left(X \cup p_{2}\right)= \begin{cases}\rho_{M}(X), & \text { se } \bar{X}^{M_{1}} \in \mathcal{F}_{2} \\
\rho_{M}(X)+1, & \text { caso contrárjo }\end{cases}
\end{aligned}
$$

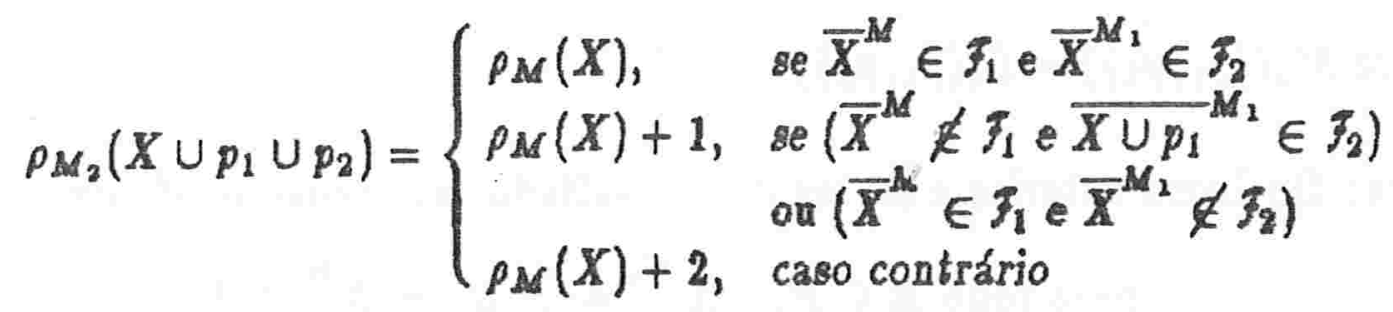

Prova: Consideremos as extensões pontuais isoladamente: 
(I) Se $X \subseteq E$ então

$$
\begin{aligned}
\rho_{M_{1}}(X) & =\rho_{M}(X) \\
\rho_{M_{1}}\left(X \cup \rho_{1}\right) & = \begin{cases}\rho_{M}(X), & \text { se } \rho_{1} \in \bar{X}^{M_{1}} \\
\rho_{M}(X)+1, & \text { caso contrário }\end{cases}
\end{aligned}
$$

(II) Se $X \subseteq E \cup p_{1}$ então

$$
\begin{aligned}
\rho_{M_{3}}(X) & =\rho_{M_{1}}(X) \\
\rho_{M_{2}}\left(X \cup p_{2}\right) & = \begin{cases}\rho_{M_{1}}(X), & \text { se } p_{2} \in \bar{X}^{M_{3}} \\
\rho_{M_{1}}(X)+1, & \text { caso conotrário }\end{cases}
\end{aligned}
$$

Combinando (I), (II) e os seguintes fatos

$$
\begin{gathered}
\text { para todo } X \subseteq E, \quad p_{1} \in \bar{X}^{M_{1}} \Leftrightarrow \bar{X}^{M} \in \bar{f}_{1} \\
\text { para todo } X \subseteq E \cup p_{1}, \quad p_{2} \in \bar{X}^{M_{2}} \Leftrightarrow \bar{X}^{M_{3}} \in \bar{f}_{2}
\end{gathered}
$$

Obtemos a expressão de $\rho_{M_{2}}$ do enunciado.

Proposicão A.2. Seja $F$ um filtro modular 2-compativel de $M$ e considere o seguinte diagrama de extensóes pontuais:

$$
M(E) \stackrel{P}{\longrightarrow} M_{1}\left(E \cup p_{1}\right) \stackrel{\left[\left.P\right|_{M_{1}}\right.}{\longrightarrow} M_{2}\left(E \cup p_{1} \cup p_{2}\right)
$$

Então $M_{2}$ é a extensão normal de $M$ sobre $E \cup p_{1} \cup_{p_{2}}$ de empilhamento

$$
\varepsilon^{(i)}=\left\{X \in \tilde{F}_{M}: p_{M_{2}}\left(X \cup p_{1} \cup p_{2}\right)-p_{M}(X)=i\right\} \quad i=0,1,2
$$

tal que $M_{2}\left(\left\{p_{1}, p_{2}\right\}\right)=\mathcal{L}\left(\left\{p_{1}, p_{2}\right\}\right)$.

Prova: Do Lema anterior e do seguinte resultado facilmente verificácel

$$
\text { para todo } X \subseteq E, \quad \bar{X}^{M_{3}} \in[\xi]_{M_{3}} \Leftrightarrow \bar{X}^{M} \in \bar{F}
$$

caracterizamos $\rho_{M}$ : 
para todo $X \subseteq E$,

$$
\begin{aligned}
\rho_{M_{2}}(X) & =\rho_{M}(X) \\
\rho_{M_{3}}\left(X \cup p_{i}\right) & = \begin{cases}\rho_{M}(X), & \text { se } \bar{X}^{M} \in \xi \\
\rho_{M}(X)+1, & \text { caso contrário } \quad i=1,2\end{cases} \\
\rho_{M_{2}}\left(X \cup p_{1} \cup p_{2}\right) & = \begin{cases}\rho_{M}(X), & \text { se } \bar{X}^{M} \in \xi \\
\rho_{M}(X)+1, & \text { se } \bar{X} \notin \xi \text { e } \overline{X \cup p_{1}} M_{1} \in[\xi]_{M_{1}} \\
\rho_{M}(X)+2, & \text { caso cortrário }\end{cases}
\end{aligned}
$$

de forma equivalente, para todo $X \subseteq E$ e $Y \subseteq\left\{p_{1}, p_{2}\right\}$

$$
\rho_{N}(X \cup Y)=\min \left(\rho_{M}(X)+|Y|, \rho_{M}(X)+i\right)
$$

onde $\bar{X}^{M} \in \mathcal{E}^{(i)}$. Portanto, $N$ é a extensão normal de $M$ sobre $E \cup p_{1} \cup$ $p_{2}$ de empilhamento $\mathcal{E}^{(0)}, \mathcal{E}^{(1)}, \mathcal{E}^{(2)}$ tal que $M_{2}\left(\left\{p_{1}, p_{2}\right\}\right)=\mathcal{L}\left(\left\{p_{1}, p_{2}\right\}\right)$ já que $\rho_{M_{2}}\left(\left\{p_{1}, p_{2}\right\}\right)=2$.

Teorema 4 . Sejam $\bar{J}_{1}$ e $\bar{\eta}_{2}$ fltros madulares de $M$ não triviaje distintos. Então são equivalentes:

i. $\tau_{1}$ e $\eta_{2}$ são fltros modulares compativeis de $M$

ii. (a) $\eta_{1} \cap \bar{\gamma}_{2}$ é um filtro modular 2-compat́fvel de $M$

(b) Seja $\varepsilon^{(0)}=\xi_{1} \cap \xi_{2}, \varepsilon^{(1)}, \varepsilon^{(2)}$ o empilhamento dos fechados de $M$ determinado pelo filtro modular 2 -compatfvel $\xi_{1} \cap \xi_{2}$. Então para todo $X, Y \in \mathcal{F}_{M}$ tais que $Y$ cobre $X, Y \in \xi_{1}-\xi_{2}$ (resp., $\xi_{3}-\xi_{1}$ ) e $X \in \mathcal{E}^{(1)}$, temos que $X \in F_{1}-\mathcal{F}_{2}$ (resp., $\xi_{2}-\xi_{1}$ )

Prova: Verifiquemos que î̀. $\Rightarrow$ i. Considere a seguinte decomposição dos fechados de $M$ :

$$
\varepsilon_{1}^{(0)}=\mathcal{E}^{(0)}=\xi_{1} \cap \xi_{2} \quad \varepsilon_{1}^{(1)}=\varepsilon^{(1)} \cup\left(\xi_{1} \Delta \xi_{2}\right) \quad \varepsilon_{1}^{(2)}=\mathcal{E}^{(2)}-\left(\xi_{1} \Delta \xi_{2}\right)
$$

Mostremos que $\mathcal{E}_{1}^{(0)}, \mathcal{E}_{1}^{(1)}, \mathcal{E}_{1}^{(2)}$ satisfazem as duas p:opriedades que caracterizam empilhamentos:

(E1) Sejam $X, Y \in \mathcal{F}_{M}$ tais que $X$ cobre $Y$ e $X \in \mathcal{E}_{1}^{(i)}$ para algum $i=1$, 2. Lembrando que $\varepsilon^{(0)}, \varepsilon^{(1)}, \varepsilon^{(2)}$ a empilhamento e usando aid.(b) é fácil verificar que $X \in \varepsilon_{1}^{(j)} \cap \varepsilon_{1}^{(i-1)}$. 
(E2) Sejam $X, Y \in \mathcal{F}_{M}$ tajs que $X, Y$ cobrem $X \cap Y$ e $X, Y, \overline{X \cup Y}^{M} \in \mathcal{E}_{1}^{(i)}$ para algum $i=0,1,2$. Se $i=0$ on 2 decorre de $(E 1)$ e das propriedades de filtro modular que $X \cap Y \in \mathcal{E}_{1}^{(i)}$. Suponhamos que $i=1$. Se $\overline{X \cup Y^{M}} \in \xi_{1}-\xi_{2}$, de $\ddot{i}$.(b) obtemos $X, Y, \overline{X \cup Y}^{M} \in \mathcal{I}_{1}-\xi_{2}$ e como $X, Y$ formam um par modular, $X \cap Y \in \mathcal{F}_{1}-\xi_{2}$. 0 caso $\overline{X \cup Y}^{M} \in \mathcal{F}_{2}-\xi_{1}$ é simétrico. Se $\overline{X \cup Y}^{M} \notin \xi_{1} \Delta f_{2}$ entz̃o $X, Y, \overline{X \cup Y}^{M} \in \mathcal{E}^{(1)}-\left(\xi_{1} \Delta \xi_{3}\right)$, logo $X \cap Y \in$ $\varepsilon^{(1)}-\left(\tilde{f}_{1} \Delta \tilde{f}_{3}\right)$.

Concluindo, em qualquer caso, $X \cap Y \in \varepsilon_{1}^{(i)}$.

Portanto $\varepsilon_{1}^{(0)}{ }_{1} \varepsilon_{1}^{(1)}, \varepsilon_{1}^{(2)}$ é um empilhamento dos fechados de $M$ com grau 2 pois $\emptyset \neq \varepsilon^{(1)} \subseteq \mathcal{E}_{1}^{(1)}$ e ${ }^{-M^{M}} \in \mathcal{E}^{(2)}-\left(\tilde{I}_{1} \Delta \mathcal{I}_{2}\right) \subseteq \mathcal{E}_{2}^{(2)}$. Seja $M^{\prime}$ a extensão normal de $M$ sobre $E \cup p_{1} \cup p_{2}$ determinada pelo empilhamento $\varepsilon_{1}^{(0)}, \varepsilon_{1}^{(1)}, \varepsilon_{1}^{(2)}$ tal que $M^{\prime}\left(\left\{p_{1}, p_{2}\right\}\right)=\mathcal{L}\left(\left\{p_{1}, p_{2}\right\}\right)$. Temos então que para todo $X \subseteq E$ e $Y \subseteq\left\{p_{1}, p_{2}\right\}$

$$
\rho_{M}(X \cup Y)=\min \left(\rho_{M}(X)+|Y|, \rho_{M}(X)+i\right)
$$

onde $\bar{X}^{M} \in \varepsilon_{1}^{(i)}$. Defino então a seguinte função sobre o conjunto das partes de $E \cup p_{1} \cup p_{2}$ :

para todo $X \subseteq E$,

$$
\begin{aligned}
\rho_{N^{\prime}}(X) & =\rho_{M}(X) \\
\rho_{N^{\prime}}\left(X \cup p_{i}\right) & = \begin{cases}\rho_{M}(X), & \text { se } \bar{X}^{M} \in \xi_{i} \\
\rho_{M}(X)+1, & \text { caso contrário } \quad i=1,2\end{cases} \\
\rho_{N}\left(X \cup p_{1} \cup p_{2}\right) & =\rho_{M}\left(X \cup p_{1} \cup p_{3}\right)
\end{aligned}
$$

Verifiquemos que $\rho_{N}$, satisfas os seguintes axiomas de posto:

(R1) $\rho_{N^{\prime}}(\emptyset)=0$

(R2) Para todo $Z \subseteq E \cup p_{1} \cup p_{2}$ e $x \in E \cup p_{1} \cup p_{2}$ temos que

$$
\rho_{N^{\prime}}(Z) \leq \rho_{N^{\prime}}(Z \cup x) \leq \rho_{N^{\prime}}(Z)+1
$$

(R3) Para todo $Z \subseteq E \cup p_{1} \cup p_{2}$ e $x, y \in E \cup p_{1} \cup p_{2}$,

$$
\begin{gathered}
\text { ge } \rho_{N^{\prime}}(Z \cup x)=\rho_{N^{\prime}}(Z \cup y)=g_{N^{\prime}}(Z) \\
\text { entẫo } \rho_{N^{\prime}}(Z \cup x \cup y)=\rho_{N^{\prime}}(Z)
\end{gathered}
$$

Note que para $i=1,2, \rho_{N}$ restrito a $E \cup p_{i}$ é a função posto da extensão pontual de $M$ pelo filtro modular $\xi_{i}$. Logo é suficiente verificar (Rq) quando 
$\left\{p_{1}, p_{2}\right\} \subseteq Z \cup x$ e $(\mathrm{R} 3)$ quando $\left\{p_{1}, p_{2}\right\} \subseteq Z \cup x \cup y$. Podemos supor também que $\left\{p_{1}, p_{3}\right\} \nsubseteq Z$ pois $p_{N}$, restrito a conjuntos que contêm $\left\{p_{1}, p_{2}\right\}$ coincide com a funçã̃o posto $\rho M$ '.

(R2) $Z=X \cup p_{1}, X \subseteq E$ e $z=p_{2}$ é o t́nico caso que necessita verificação. Se $\bar{X}^{M} \in \mathcal{F}_{1}$ então $\bar{X}^{M} \in \mathcal{E}_{1}^{(0)} \cup \mathcal{E}_{1}^{(1)}, \log \circ$

$$
\rho_{N^{\prime}}(Z)=\rho_{M}(X) \leq \rho_{N^{\prime}}(Z \cup x) \leq \rho_{M}(X)+1 \leq \rho_{N^{\prime}}(Z)+1
$$

Por outro lado, se $\bar{X}^{M} \notin F_{1}$ então $\bar{X}^{M} \in \varepsilon_{1}^{(1)} \cup \varepsilon_{1}^{(2)}$, logo

$$
\rho_{N^{\prime}}(Z)=\rho_{M}(X)+1 \leq \rho_{N^{\prime}}(Z \cup x) \leq \rho_{M}(X)+3=\rho_{N^{\prime}}(Z)+1
$$

(R3) Seja $Z \subseteq E \cup p_{1} \cup p_{2}$ e $x, y \in E \cup p_{1} \cup p_{2}$ tais que $\left\{p_{1}, p_{2}\right\} \subseteq Z \cup x \cup y$, $\left\{p_{1}, p_{2}\right\} \nsubseteq Z$ e $p_{N}(Z \cup x)=p_{N}(Z \cup y)=p_{N}(Z)$. Temos então duas possibilidades:

Caso 1: $\mathbb{Z} \subseteq E, x=p_{1}$ e $y=p_{2}$

Entäo para cada $i=1,2$ temos

$$
\rho_{N^{\prime}}\left(Z \cup p_{i}\right)=\rho_{N^{\prime}}(Z)=\rho_{M}(Z)
$$

Logo $\bar{Z}^{M} \in F_{1} \cap F_{2}$ e portanto

$$
\begin{aligned}
\rho_{N}(Z \cup x \cup y) & =\rho_{M}(Z \cup x \cup y) \\
& =\rho_{M}(Z)=\rho_{N}(Z)
\end{aligned}
$$

Caso 2: $\mathbb{Z}=X \cup p_{1}, X \subseteq E, x \in E, y=p_{2}$ (ou trocando $p_{1}$ com $p_{2}$ )

Se $x \in \bar{X}^{M}$ então

$$
\rho_{N^{\prime}}(Z \cup \mathscr{Z} \cup y)=\rho_{N^{\prime}}(Z \cup y)=\rho_{N^{\prime}}(Z)
$$

Suponhamos que $x \notin \bar{X}^{M}$, nesse caso, $p_{N}(Z \cup x)=\rho_{N}(Z)$ e $\rho_{M}(X \cup Z)=\rho_{M}(X)+1$ implicam que $\bar{X}^{M} \notin \xi_{1}$, donde $\rho_{N^{\prime}}(Z)=$ $\rho_{M}(X)+1$ e $\overline{X \cup X} \bar{X}^{M} \in \mathcal{F}_{1}$. Como $\rho_{N}(\mathbb{Z})=\rho_{N^{\prime}}\left(\mathbb{Z} \cup p_{2}\right)$ então $\bar{X}^{M} \in \mathcal{E}^{(1)} \cup\left(\mathcal{F}_{1} \Delta \mathcal{F}_{2}\right)=\mathcal{E}_{1}^{(1)}$. Combinando com o fato de que $\bar{X}^{M} \notin \xi_{1}$ tem-se que $\bar{X}^{M} \in \mathcal{E}^{(1)} \cup\left(\bar{f}_{2}-\xi_{1}\right)$. Se $\bar{X}^{M} \in \mathcal{F}_{3}-\bar{F}_{1}$ é claro que $\overline{X \cup X} \bar{x}^{M} \in \xi_{1} \cap \xi_{2}$. Por outro lado, se $\bar{X}^{M} \in \mathcal{E}^{(1)}$ usando a propriedade (EI) de empilhamento temos que $\overline{X \cup x^{M}} \in$ 
$\varepsilon^{(0)} \cup \mathcal{E}^{(1)} ; \overline{X \cup x}^{M} \notin \varepsilon^{(1)}$ pois caso contrário teŕanos $\overline{X \cup x}^{M} \in$ $\xi_{1}-\xi_{2}, \bar{X}^{M} \in \varepsilon^{(1)}$ e $\bar{X}^{M} \notin \xi_{1}-\xi_{2}$ o que contradis $i$ i. (b). Logo $\overline{X \cup x}^{M} \in \xi_{1} \cap \xi_{2}$. Então

$$
\begin{aligned}
\rho_{N^{\prime}}(Z \cup x \cup y) & =p_{N^{\prime}}\left(X \cup p_{1} \cup p_{2} \cup x\right) \\
& =p_{M}(X \cup x)=p_{N^{\prime}}(Z)
\end{aligned}
$$

Seja $N^{\prime}$ a extensão de $M$ sobre $E \cup p_{1} \cup p_{2}$ determinada por $p N^{\prime}$. E claro que

$$
M(E) \stackrel{\xi_{i}}{\longrightarrow} N^{\prime}\left(E \cup p_{i}\right) \quad i=1,2
$$

portanto $f_{1}$ e $\xi_{2}$ sa filtros modulares compativeis de $M$.

Mostremos agora que $i_{.} \Rightarrow 8 i$. Suponha que $f_{1}$ e $\xi_{2}$ são compaitiveis; então pela Proposição 3.4. temos o seguinte diagrama comutativo:

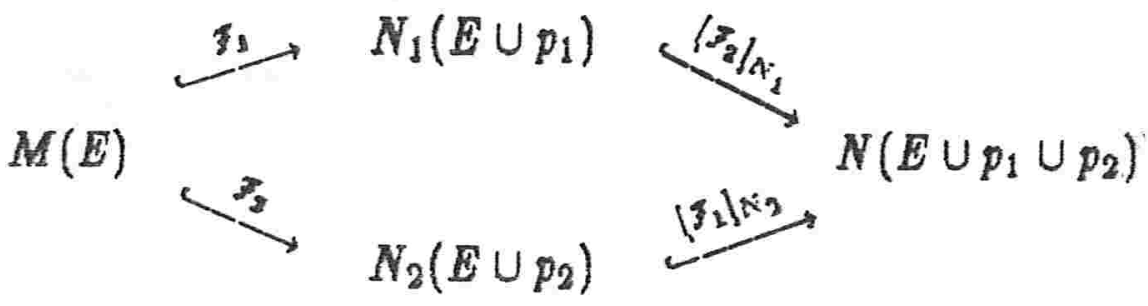

$\log 0 p_{N}\left(\left\{p_{1}, p_{2}\right\}\right)=3$ pois se $p_{N}\left(\left\{p_{1}, p_{2}\right\}\right)=0$ então $p_{1}=\eta_{M}=\xi_{2}$, e se $\rho_{N}\left(\left\{p_{1}, p_{3}\right\}\right)=1$, como $\rho_{N}\left(p_{i}\right)=1$ para $i=1,2$, então $\left[\tilde{p}_{2}\right]_{N_{1}}=\left[\bar{p}_{1}^{N_{3}}\right] \mathrm{e}$ $\left[\xi_{1}\right]_{N_{2}}=\left[{\overline{p_{2}}}^{N_{2}}\right]$ donde $\xi_{1}=\xi_{2}$, um absurdo. Sejam $\varepsilon_{1}^{(0)}=\xi_{1} \cap \xi_{2}, k_{1}^{(1)}, \varepsilon_{1}^{(2)} 0$ empilhamento da extensão $N$ de $M$, e $N^{\prime}$ a extensão normal de $M$ sobrit $\cup_{p_{1}} \cup p_{2}$ determinada por esse empilhamento, onde $N^{\prime}\left(\left\{p_{1}, p_{3}\right\}\right)=\mathcal{L}\left(\left\{p_{1}, p_{3}\right\}\right)$. Tembrando a Proposição 2.11. não é diffcil verificar que temos o seguinte diagrama:

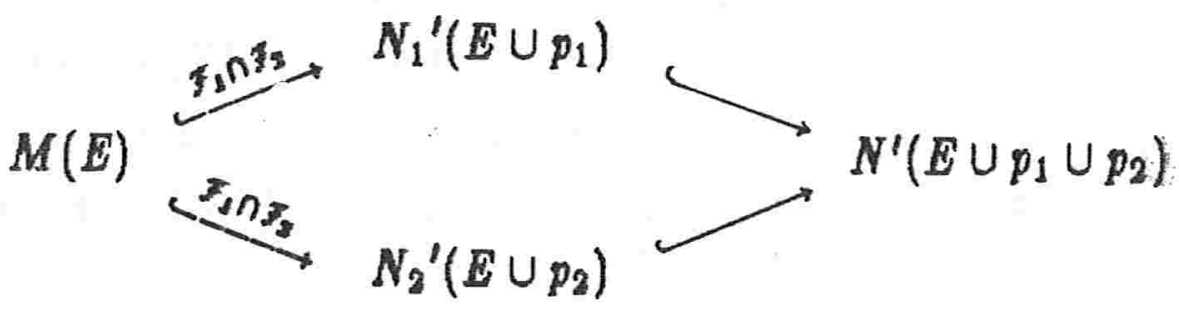


Logo, Be

$$
M(E) \stackrel{\xi_{1} \cap \xi_{2}}{\longrightarrow} M_{1}\left(E \cup p_{1}\right) \stackrel{\left[\xi_{1} \cap F_{2}\right]_{M_{3}}}{\longrightarrow} M_{2}\left(E \cup p_{1} \cup p_{2}\right)
$$

pela minimalidade de $M_{2}$ em $\Phi\left(\xi_{1} \cap \xi_{2}, \xi_{1} \cap \xi_{2}\right)$ já observada no final da seção anterior, temos que $p_{M_{2}}\left(\left\{p_{1}, p_{2}\right\}\right) \geq p_{N^{\prime}}\left(\left\{p_{1}, p_{2}\right\}\right)=2$. Portanto $\xi_{1} \cap \xi_{2}$ é 2-compativel, donde $i \dot{i}$.(a). Seja então $\mathcal{E}^{(0)}=\xi_{1} \cap \xi_{2}, \varepsilon^{(1)}, \mathcal{E}^{(2)}$ o empilhamento de fechados de $M$ determinado pelo filtro 2 -compativel $\xi_{1} \cap \xi_{2}$. E fácil verificar que $\mathcal{E}^{(1)} \subseteq \mathcal{E}_{1}^{(1)}$ usando o fato de que $\rho_{M_{2}}(Z) \geq \rho_{N^{\prime}}(Z)$ para todo $Z \subseteq E \cup p_{1} \cup p_{2}$. Verifiquemos que vale $\ddot{i}$.(b). Sejam $X, Y \in \mathcal{F}_{M}$ tais que $Y$ cobre $X, X \in \mathcal{E}^{(1)}$ e $Y \in \tilde{F}_{1}-\xi_{2}$ (a veríicação do caso $Y \in \mathcal{F}_{2}-\xi_{1}$ é análoga). Suponha por absurdo que $X \notin \xi_{1}-\xi_{2}$. Como $X \subseteq Y$ e $X \in \varepsilon^{(1)}$ então $Y \in \varepsilon^{(1)} \cup \varepsilon^{(0)} ;$ mas $Y \notin \mathcal{F}_{2} \supseteq \varepsilon^{(0)}$, logo $Y \in \mathcal{E}^{(1)}$. Portanto $X, Y \in \mathcal{E}_{1}^{(1)}$, donde $\rho_{N}\left(X \cup p_{1} \cup p_{2}\right)=\rho_{M}(X)+1 \mathrm{e}$ $\rho_{N}\left(Y \cup p_{1} \cup p_{3}\right)=\rho_{M}(Y)+1$. Chegamos então à seguinte contradição:

$$
p_{3} \notin \bar{Y}^{N_{1}} \text { e } p_{3} \in{\overline{X \cup p_{1}}}^{N_{1}} \subseteq \bar{Y}^{N_{1}}
$$

Embora a técnica da demonstração deste resultado em alguns pontos seja. praticamente análoga àquela do Teorema 3.1., pareceu-nos importante registrarmos todos os passos para salientarmos as diferenças existentes entre 08 dois resultados.

Deixamos registrados a seguir vários corolários interessantes do Teorema 4.3.. Muitos deles são na realidade Corolários da prova do Teorema 4.3..

Corolério 4.4. Sejam $\xi_{1}$ e $\xi_{2}$ filtros modulares de $M$. Ența $\xi_{1}$ e $\xi_{2}$ são compativeis se e somente se $\tau_{1}, \tau_{1} \cap \pi_{2}$ e $\tau_{2}, \tau_{1} \cap T_{2}$ sắ pares de filtros modulares compativeis de $M$.

Prova: Podemos supor que $\xi_{1}, \xi_{2}$ são não triviais e que $\xi_{i} \neq \xi_{1} \cap \xi_{3}$ para $i=1,2$ pois ocorrendo alguma dessas situaçōes a equivalência acima torna-se trivial.

Suponhamos que $F_{1}, F_{2}$ são compativeis. Nesse caso fácil ver que a condição ii. dc Teorema 4.3, está verificada para os pares $\xi_{1}, f_{1} \cap \xi_{2}$ e $F_{3}, f_{1} \cap \xi_{2}$ de filtros modulares de $M$ já que $\xi_{1} \cap\left(\xi_{1} \cap \xi_{2}\right)=\xi_{2} \cap\left(\xi_{1} \cap \xi_{2}\right)=\xi_{1} \cap \xi_{2}$. Logo $\xi_{1}, \xi_{1} \cap \xi_{2} \in \xi_{2}, \tilde{f}_{1} \cap \xi_{3}$ são compatfveis.

De outro lado, suponhamos que $\xi_{1}, \xi_{1} \cap \xi_{2}$ \& $\xi_{2}, \xi_{1} \cap \xi_{2}$ são compatíveis.

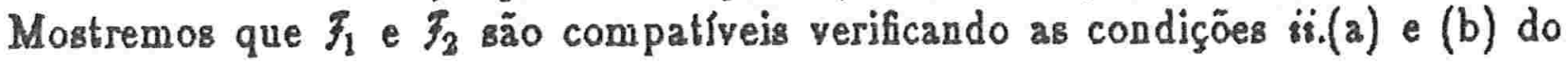


Teorema 4.3. E claro que vale $i .(a)$, e $\ddot{i}$.(b) segue do fato de que $\xi_{1}-F_{2}=$ $\xi_{1}-\left(\xi_{1} \cap \xi_{2}\right)$ e $\xi_{2}-\xi_{1}=\xi_{2}-\left(\xi_{1} \cap \xi_{2}\right)$.

Em [Ch], Cheung apresenta uma prova para o resultado acima, dentro do contexto de subclasses lineares.

Lema 1.5 . Seja $\xi$ um filtro modular de $M$ tal que $\xi \neq \xi_{M}$. Se $\xi 1$ e $\xi$ são filtros modulares ultra-compativeis de $M$ tajs que $\xi=\xi 1=\xi "$, então $\xi$ \& $z$-compativel ou é um flitro principal.

Prova: Considere o seguinte diagrama de extenãos pontuais:

$$
M(E) \stackrel{?}{\longrightarrow} M_{1}\left(E \cup p_{1}\right) \stackrel{\left[I_{M_{1}}\right.}{\longrightarrow} M_{2}\left(E \cup p_{1} \cup p_{2}\right)
$$

Como $\neq \neq \xi_{M}$ é claro que $1 \leq \rho_{M_{2}}\left(\left\{p_{1}, p_{2}\right\}\right) \leq 2$. Se $\xi$ é 2 -compativel, não há nada a demonstrar. Suponhamos então que $\rho_{M_{2}}\left(\left\{p_{1}, p_{3}\right\}\right)=1$. Nesse caso é fácil ver que $\left[f_{M_{1}}=\left[\bar{p}_{1}^{M_{1}}\right]\right.$. Usando a ultra-compatibilidade de $\xi^{\prime}, \xi^{\prime \prime}$ obtemos ${\overline{p_{1}}}^{M_{1}} \cap E \in \bar{F}$ Mostremos que

$$
\xi=\left\{X \in \bar{I}_{M}: \bar{p}_{1}^{M_{2}} \cap E \subseteq X\right\}
$$

A inclusão $\supseteq$ é trivial. Verifiquemos a inclusão contrária. Tome $X \in \mathcal{F}$. E claro que $\bar{X}^{M_{1}} \in[\xi]_{M_{1}}, \log 0{\overline{p_{1}}}^{M_{1}} \subseteq \bar{X}^{M_{1}}$, donde ${\overline{p_{1}}}^{M_{1}} \cap E \subseteq \bar{X}^{M_{1}} \cap E=X$. Portanto Ié um filtro modular principal.

Considere um filtro modular do matroide $M$ sobre o conjunto $E$ tal que F, F são ultra-compatíveis. Os seguintes exemplos mostram que no Lema 4.5. a 2-compatibilidade de $\tilde{f}$ não exclui a possibilidade de $f$ ser principal (e vice-versa):

(1) $E=\{a, b\}, M(E)=\mathcal{L}(E), \xi=\{E\}$ é z-compatível e principal

(2) $E=\{a, b, c\}, M(E)=\mathcal{L}(E), \xi=[a]=\{a, a b, a c, w b c\}$ é principal mas não \& \&-compativel

(3) $E=\{a, b, c, d, f, g\}, M(E)=\mathcal{L}_{\delta}(E), \xi=[a b c d$, defg] $=\{a b c d$, def $g$, abedefg $f$ é 2-compattrel mas uão é principal 
Corolário 4.6. Sejam $\xi_{1}$ e $\xi_{2}$ flttros modulares de $M$ não triviais. Se $\xi$ e $\xi "$ são Gitros modulares ultra-compatfveis de $M$ onde $\xi_{1} \cap \xi_{2}=\xi^{\prime}=\xi^{\prime \prime}$ então $\xi_{1}$ e $\xi_{2}$ são filtros modulares compat́fveis de $M$.

Prova: Pelo Lema anterior distingüimos dois casos:

Caso 1: $\xi_{1} \cap \boldsymbol{\gamma}_{2}$ é filtro modular principal de $M$

Pela Proposição 1.2. do Capítulo I, $\xi_{1}, \xi_{1} \cap \xi_{2}$ e $\xi_{2}, \xi_{1} \cap \xi_{2}$ são pares de filtros modulares ultra-compatfveis e portanto compativeis. Usando 0 Corolário anterior concluímos que $\xi_{1}$ e $\xi_{2}$ são compatfveis.

Caso 2: $f_{1} \cap f_{2}$ é um filtro modular 2-compatível de $M$

Temos então a condição ï.(a) do Teorema 4.3. verificada. Mostremos também que vale ï.(b). Seja $\varepsilon^{(0)}=f_{1} \cap \xi_{2}, \varepsilon^{(1)}, \varepsilon^{(3)}$ o empilhamento de $M$ determinado pelo filtro modular 2-compativel, $\xi_{1} \cap \xi_{2}$. Afirmo que se $X \in \mathscr{F}_{M}$, uma condição necessária e suficiente para que $X \in \mathcal{E}^{(1)}$ é que $X \notin F_{1} \cap F_{3}$ e exista $Z \in F_{1} \cap \xi_{2}$ tal que $Z$ cobre $X$. A suficiência segue dos seguintes fatos:

$$
\text { se } X \notin f_{1} \cap F_{2}=\mathcal{E}^{(0)} \text { entz̃o } X \in \mathcal{E}^{(1)} \cup \mathcal{E}^{(2)}
$$

se $Z$ cobre $X$ e $Z \in \mathcal{F}_{1} \cap \mathcal{F}_{2}=\mathcal{E}^{(0)}$ então $X \in \mathcal{E}^{(0)} \cup \mathcal{E}^{(1)}$

Verifiquemos a necessidade. Considere o seguinte diagrama de extensōes pontuais

$$
M(E) \stackrel{\xi_{1} \cap \xi_{2}}{\longrightarrow} M_{1}\left(E \cup p_{1}\right) \stackrel{\left[\xi_{1} \cap \xi_{3}\right]_{M_{1}}}{\longrightarrow} M_{2}\left(E \cup p_{1} \cup p_{2}\right)
$$

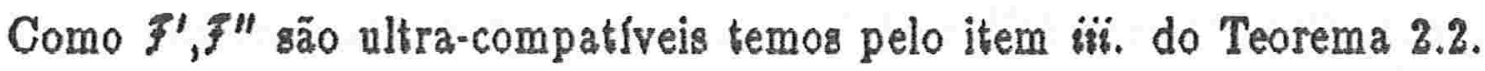
do Capítulo I que

$$
\rho_{M_{8}}\left(X \cup p_{1} \cup p_{2}\right)=\min _{X \subseteq \underline{Z} \subseteq E}\left\{\rho_{M}(Z)+\left|\left(X \cup p_{1} \cup p_{3}\right)-\bar{Z}^{M_{2}}\right|\right\}
$$

Por outro lado, $X \in \mathcal{E}^{(1)}, \log 0 \rho_{M_{2}}\left(X \cup p_{1} \cup p_{2}\right)=\rho_{M}(X)+1$. Combinando as duas expressões de $\rho_{M_{2}}\left(X \cup p_{1} \cup p_{2}\right)$ acima concluimos que existe $Z \in \mathcal{J}_{1} \cap \xi_{2}$ tal que $Z$ cobre $X$. Portanto, para todos $X, Y \in \mathcal{J}_{M}$ tais que $Y$ cobre $X, X \in \mathcal{E}^{(1)}$ e $Y \in F_{1}-F_{2}$ temos que $X \in F_{1}-F_{2}$ já que existe $Z \in \mathcal{F}_{1} \cap \mathcal{F}_{2}$ com $Z$ cobrindo $X$ e $Y \neq Z$. Se $Y \in \xi_{2}-\xi_{1}$ então por simetria $X \in \xi_{2}-F_{1}$.

Logo $\Psi_{1}, \mathcal{F}_{2}$ são filtros modulares compativeis de $M$. 
Corolário 4.7. Sejam $\xi_{1}, \xi_{2}$ filtros modulares compatfveis de $M$ não triviais, e $N$ a extensão de $M$ sobre $E \cup p_{1} \cup p_{2}$ dada pelo seguinte diagrama comutativo

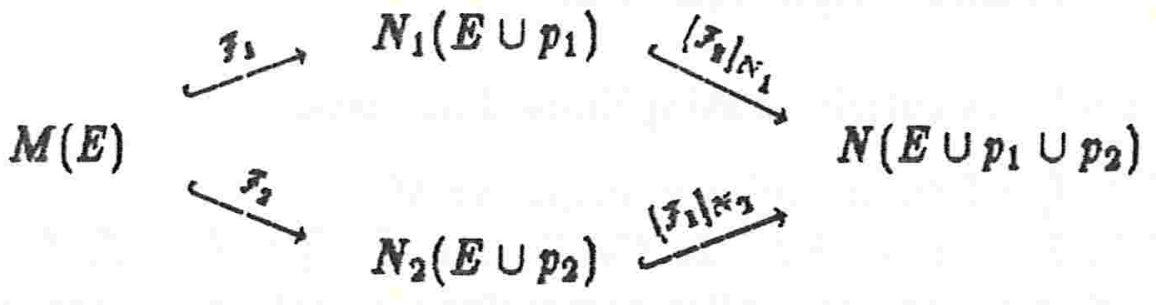

Seja também $\mathcal{E}^{(0)}=\xi_{1} \cap \xi_{2}, \mathcal{E}^{(1)}, \mathcal{E}^{(2)}$ o empilhamento de $M$ determinado pelo flitro modular 2 -compativel $\tilde{n}_{1} \cap F_{2}$. Temos então que para todo $X \subseteq E$ e $Y \subseteq$ $\left\{p_{1}, p_{2}\right\}$

$$
\rho_{N}(X \cup Y)=\min \left(\rho_{M}(X)+\mid Y-\left\{\rho_{j}: \bar{X}^{M} \in F_{j}\right\} l_{,} \rho_{M}(X)+i\right)
$$

onde $\bar{X}^{M} \in \varepsilon^{(i)}$.

Prova: Seja $\varepsilon_{1}^{(0)}=\mathcal{F}_{1} \cap \mathcal{F}_{2}, \varepsilon_{1}^{(1)}, \varepsilon_{1}^{|2|}$ o empilhamento da extensão $N$ de $M$. Seja também a extensão $N^{\prime}$ de $M$ sobre $E \cup p_{1} \cup p_{2}$ de empilhamento $\varepsilon_{2}^{(0)}=$

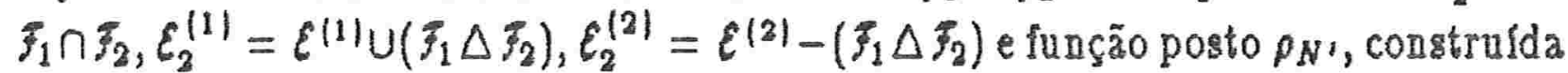
na demonstração da implicação $i i . \Rightarrow i$. do Teorema 4.3.. $\mathrm{Na}$ prova desse mesmo Teorema, ficon claro que

$$
\begin{aligned}
& \text { (I) } \mathcal{E}^{(1)} \subseteq \mathcal{E}_{1}^{(1)} \\
& \text { (II) } \rho_{N}(X) \leq \rho_{N}(X) \text { para todo } X \subseteq E \cup \eta_{1} \cup \rho_{2}
\end{aligned}
$$

De (I) e do fato de que $\xi_{1} \Delta \mathcal{F}_{2} \subseteq \mathcal{E}_{1}^{(1)}$ temos $\varepsilon_{2}^{(1)} \subseteq \mathcal{E}_{1}^{(1)}$. Portanto, combinando com (II) obtemos $\varepsilon_{2}^{(i)}=\varepsilon_{1}^{(i)}$ para $i=0,1,2$ donde concluimos que $N^{\prime}=N$. Observe que para todo $X \subseteq E$ e $Y \subseteq\left\{p_{1}, p_{2}\right\}$ a expressão de $\rho_{N}$ do enunciado do Teorema é uma forma equivalente para a expressão de $\rho_{N^{\prime}}$ dada na prova do Teorema 4.3.

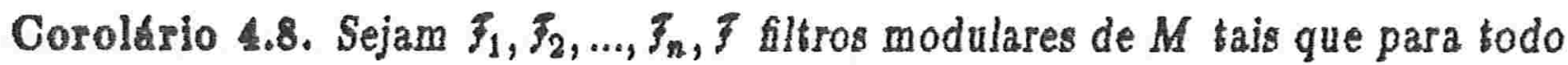
$i=1,2, \ldots, n, \xi \subseteq \xi_{i}$ e $\bigcap_{1 \leq j \leq n} \xi_{j} \neq \mathcal{F}$. Se para todo $i=1,2, \ldots, n, \xi_{i}, \xi$ são filtros modulares compsífveis de $M$ então para todo $I \subseteq\{1,2, \ldots, n\}, \bigcap_{j \in I} \xi_{j}$, são filtros modulares compat́fveis de $M$.

Prova: Suponha que $\xi_{i}, \xi$ são compativeis para todo $i=1,2, \ldots, n$ e seja $I \subseteq$ $\{1,2, \ldots, n\}$ arbitrário. Se $\xi=\xi_{M}$ ou $\bigcap_{j \in I} \xi_{j}=\xi_{M}$ é claro que $\bigcap_{j \in I} \xi_{j}, \xi$ 
são ultra-compatíveis e em particular compatíveis. Se $\bigcap_{j \in I} \xi_{j}=\xi_{\text {, trivialmente }}$ $\bigcap_{j \in I} \xi_{j}, \xi$ são compatíveis. Podemos supor então que $\xi \neq \xi_{M} \neq \bigcap_{j \in I} \xi_{j} \neq \xi ;$ nesse caso, para concluirmos que $\mathcal{F}_{1} \bigcap_{j \in I} \mathcal{F}_{j}$ são compatíveis, mostraremos que a condição ii. do Teorema 4.3 está satisfeita. E claro que $\bar{F}=\left(\bigcap_{j \in I} \xi_{j}\right) \cap \xi$ é 2-compatível, já que $\xi=\xi \cap \xi$ para todo $i=1,2, \ldots, n$, portanto $i i$.(a) está verificada. A condição $i i$ (b) segue da compatibilidade de $\tau_{i}, z$ para $i=1,2, \ldots, n$ e dos seguintes fatos

$$
\text { se } X \in \bar{F}_{j}-\mathcal{F} \text { para todo } j \in I \text { então } X \in\left(\bigcap_{j \in I} \xi_{j}\right)-\xi
$$

se existe $i \in I$ tal que $X \in \mathcal{I}-\xi_{i}$ entz̃o $X \in \mathcal{F}-\left(\bigcap_{j \in I} \tilde{\xi}_{j}\right)$

No próximo Corolário obtemos uma caracterização das extensões $N$ de $M$ sobre $E \cup P$ tais que $N(P)$ é uma linha sem laços e sem elementos paralelos.

Corolario 4.8 . Sejam $\xi_{1}, \xi_{2}, \ldots, \eta_{n}$ flltros modulares de $M$ tajs que para todos $i, j \operatorname{com} 1 \leq i<j \leq n, \boldsymbol{I}_{i} \neq \bar{\xi}_{j}$ e $\bar{\xi}_{i} \neq \xi_{M}$. São equivalentes:

i. Existe uma extensão $N$ de $M$ sobre $E \cup\left\{p_{1}, p_{2}, \ldots, p_{n}\right\}$ tal que

$$
N\left(\left\{p_{1}, p_{2}, \ldots, p_{n}\right\}\right)=\mathcal{L}_{2}\left(\left\{p_{1}, p_{2}, \ldots, p_{n}\right\}\right)
$$

e para todo $i=1,2, \ldots, n$,

$$
M(E) \stackrel{\bar{\gamma}_{i}}{\longrightarrow} N\left(E \cup p_{i}\right)
$$

ii. (a) $f_{1}, f_{2}, \ldots, \xi_{n}$ são dois a dois compativeis

(b) $\xi_{i} \cap \xi_{j}=\xi_{1} \cap F_{2} \cap \ldots \cap \xi_{n}$ para bodos $i, j \operatorname{com} 1 \leq i<j \leq n$

Prova: É fácil ver que $i \Rightarrow$ i.; observe que para todos $i, j \operatorname{com} 1 \leq i<j \leq n$, $\left\{p_{1}, p_{3}, \ldots, p_{n}\right\} \subseteq{\overline{\left\{p_{i}, p_{j}\right.}}^{N}$ e portanto

$$
\begin{aligned}
\xi_{i} \cap \xi_{j} & =\left\{X \in q_{M}: p_{N}\left(X \cup p_{i} \cup p_{j}\right)=\rho_{M}(X)\right\} \\
& =\left\{X \in \xi_{M}: p_{N}\left(X \cup\left\{p_{1}, p_{2}, \ldots, p_{n}\right\}\right)=p_{M}(X)\right\} \\
& =\xi_{1} \cap \xi_{2} \cap \ldots \cap \xi_{n}
\end{aligned}
$$


A prova da implicação ii. $\Rightarrow$ i. é semelhante à prova da mesma implicação do Teorema 4.3. ; tomando $\xi=\xi_{1} \cap \xi_{2} \cap \ldots \cap \xi_{n}$ temos por ii.(a) e (b) que $\xi$ é 2-compatível. Imitando a demonstração do Teorema 4.3., provamos que

- $\varepsilon_{1}^{(0)}=\mathcal{E}^{(0)} \varepsilon_{1}^{(1)}=\mathcal{E}^{(1)} \cup\left(\bigcup_{1 \leq i \leq n} \xi_{i}-\xi\right) \quad \varepsilon_{1}^{(2)}=\varepsilon^{(3)}-\left(\bigcup_{1 \leq i \leq n} \xi_{i}-\right.$ 7) é um empilhamento de $M$, onde $\varepsilon^{(0)}, \varepsilon^{(1)}, \varepsilon^{(2)}$ é o empilhamento determinado pelo filtro modular 2-compativel $\xi$.

- A função $\rho_{N}$ sobre o conjunto das partes de $E \cup\left\{p_{1}, p_{2}, \ldots, p_{n}\right\}$ definida por:

para todo $X \subseteq E$ e $Y \subseteq\left\{p_{1}, p_{2}, \ldots, p_{n}\right\}$ tal que $|Y| \geq 2$,

$$
\begin{aligned}
\rho_{N}(X) & =\rho_{M}(X) \\
\rho_{N}\left(X \cup p_{i}\right) & = \begin{cases}\rho_{M}(X), & \text { se } \bar{X}^{M} \in \mathcal{I}_{i} \\
\rho_{M}(X)+1, & \text { caso contrário } \quad i=1,2, \ldots, n\end{cases} \\
\rho_{N}(X \cup Y) & =\rho^{\prime}(X \cup Y)
\end{aligned}
$$

satisfaz os axiomas de posto, onde $\rho^{\prime}$ é a função posto da extensão normal $N^{\prime}$ de $M$ sobre $E \cup\left\{p_{1}, p_{3}, \ldots, p_{n}\right\}$ determinada pelo empilhamento $\varepsilon_{1}^{(0)}, \varepsilon_{1}^{(1)}, \varepsilon_{1}^{(2)}$ tal que $N^{\prime}\left(\left\{p_{1}, p_{2}, \ldots, p_{n}\right\}\right)=\mathcal{L}_{2}\left(\left\{p_{1}, p_{2}, \ldots, p_{n}\right\}\right)$.

Portanto o matróide $N$ com a funçăo posto $\rho_{N}$ verifica i...

Imitando a prova do Corolário 4.7. obtemos a seguinte expressão para a função posto do matróide $N$ do item i. do Corolário anterior:

para todo $X \subseteq E$ e $Y \subseteq\left\{p_{1}, p_{2}, \ldots, p_{n}\right\}$,

$$
\rho_{N}(X \cup Y)=\min \left(\rho_{M}(X)+\left|Y-\left\{p_{j}: \overline{\boldsymbol{X}}^{M} \in \bar{\xi}_{j}\right\}\right|, \rho_{M}(X)+i\right)
$$

onde $\bar{X}^{M} \in \mathcal{E}^{(i)}$ e $\mathcal{E}^{(0)}, \mathcal{E}^{(1)}, \mathcal{E}^{(2)}$ É o empilhamento de $M$ deferminado pelo filtro modular 2-compatível $\xi_{1} \cap \xi_{2} \cap \ldots \cap \xi_{m}$. 

Capitnlo III

\section{Compatibilidade de Subclasses Lineares}

Usualmente, extensöes pontuais de matróides são caracterizad as em termos de filtros modulares de fechados. Neste capitulo apresentaremos uma caracterigação equivalente bastante conhecida, em termos de subclasses lineares, introdusida inicialmente por Crapo em 1965. A partir dela, estabeleceremos uma condição necessária e guficiente para que dois filtros modulares comparáveis por inclusão sejam compatíveis. Com isso conseguiremos na realidade uma caracterişąão para todos os pares de filtros modulares compatíveis, bastando lembrar o Corolário 4.4. do Capitulo II, que redus o problema da compatibilidade de pares de filtros modulares quaisquer para pares de filtros modulares comparáveis.

$\mathrm{Na}$ primeira seção, além de examinarmos a relação existente entre as sub. classes lineares e os filtros modulares de um matróide, apresentaremos uma construção seyuencial natural de subclasses lineares, ferramenta importante a ser utilisada posteriormente.

A última seção será inteiramente dedicada a problema da compatibilidade de pares de subclasses lineares comparáveis por inclusão. 


\section{A RELACIOO RNTRE SUBCLASSES LIN RARES}

E FILTROS MODULARES

Dado um filtro modular $\$$ de $M$, concentremos nossa atenção nos hiperplanos de $\bar{\xi}$. Sejam $X, Y$ e $Z$ hiperplanos distintos tais que $X, Y \in \mathcal{Z}$ e $X, Y$, $\boldsymbol{Z}$ cobrem $X \cap Y \cap \boldsymbol{Z}$. Nesse caso não é difficil verificar que $X \cap Y=X \cap Y \cap Z$. Logo $X, Y$ formam um par modular, donde concluimos que $X \cap Y \in F$. Mas então $Z \in \mathcal{Z} \in$ já que $Z$ cobre $X \cap Y=X \cap Y \cap Z$. Veremos nesta seção que essa propriedade é sutciente para caracteriar os filtros modulares.

No que se segue $\forall$ denota a famflia dos hiperplanos ou copontos de $M$. Diremos que $X, Y, Z \in X$ distintos formam uma tripla geradora se $X, Y, Z$ cobrem $X \cap Y \cap \mathbb{Z}$, ou o que é equivalente, se $X \cap Y \cap \mathbb{Z}$ é uma colinha de $M$.

Estamos agora em condiçōes de defnir subclasses lineares. Uma subfamflia $S$ de $V$ é uma subclasse linear de $M$ se para toda tripla geradora $X, X, Z$ tal que $X, Y \in S$ temos também que $Z \in S$.

No inf́cio da seçá ficon claro que ae $I$ é nm filtro modular de $M$, então $\xi \cap K$ é uma subclasse linear. Uma pergunta natural que imediatamente nos ocorre, é se a partir de uma subclasse linear podemos obter um filtro modular de uma naneira assim tão simples. A resposta é dada pelo próximo Lema.

Lema 1.1. Seja $S$ uma subclasse linear de $M$ e considere o conjunto $\$$ dos fechados $X$ de $M$ tais que todo hiperplano contendo $X$ está em $S$. Temos então que $F$ é um filtro modular de $M$.

Prova: Por definição, se $X, Y \in \mathcal{F}_{M}, X \in \xi$ e $X \subseteq Y$ então $Y \in \xi$. Agora, suponha por absurdo que existem $X, Y \in \xi$ par modular tais que $X \cap Y \notin \xi$. Pela hipótese, $\rho_{M}(X \cap Y)<\rho M-2$. Seja $\mathbb{H} \in X-S$ tal que $X \cap Y \subseteq H$, e seja $Z \in \bar{J}_{M}$ tal que $X \cap Y \subseteq Z$ e $H$ cobre $Z$. t fácil ver que $\overline{X \cup Z}^{M}, \overline{Y \cup Z}^{M} \in S$ e $\overline{X \cup Z}^{M} \cap \overline{Y \cup Z}^{M} \cap H=\mathbb{Z}$. Mas então $H \in S$, uma contradição. Portanto $z^{2}$ um filtro modular de $M$.

Toda essa discussão inicial nos permite finalmente estabelecer a bijeção entre subclasses lineares e filtros modulares.

Proposição 1.2. A função que a cada subclasse linear $S$ de $M$ associa o filtro modular dado pelo Lema 1.1., é uma bijeção entre a famflia das subclasses lineares e a famlia dos flitros modulares de $M$. 
Prova: 0 filtro modular $\xi$ dado pelo Lema 1.1. pode aer descrito da seguinte forma

$$
\bar{\xi}=\left\{X \in \xi_{M}: \text { se } X \subseteq Y \text { e } Y \in \forall \text { então } Y \in S\right\}
$$

E evidente que $S=\xi \cap K$, donde se conclui que a função acima é injetora. Para mostrarmos que ela também é sobrejetora, basta provarmos que dado um filtro modular $F$ de $M$, ele coincide com o geguinte filtro modular

$$
\xi_{1}=\left\{X \in \bar{\xi}_{M}: \text { se } X \subseteq Y \text { e } Y \in X \text { entz̃o } Y \in\{\cap K\}\right.
$$

isto $\hat{e}, \xi$ é imagem da subclasse linear $\xi \cap X$.

Seja $X \in \bar{F}$. Se $X \subseteq Y$ $Y \in \forall$ é claro que $Y \in Z \cap K$ pois $z$ filtro modular. Logo $\xi \subseteq \xi_{1}$.

Agora suponhamos por absurdo que existe $X \in \xi_{1}-\xi$. Posso tomar $X$ de maior posto possivel com essa propriedade. Temos que $\rho_{M}(X)<\rho M-1$ pois caso contrário $X \in(\xi \cap X) \cup E \subseteq \xi$, uma contradição. Tome $Y \in \xi_{1}$ tal que $Y$ cobre $X$. Pela maximalidade do posto de $X$ em relação a elementos de $\xi_{1}-\xi$, devemos ter $Y \in \mathcal{F}$. Um raciocínio simples mostra que existe $H \in X$ tal que $H \cap Y=X$. Note que $H \in T$ já que $X \in I_{1}$ e $X \subseteq H \in X$. Temos também que

$$
\begin{aligned}
\rho_{M}(H)+\rho_{M}(Y) & =\rho_{M}(H)+\rho_{M}(X)+1 \\
& =\rho_{M}+\rho_{M}(X) \\
& =\rho_{M}(H \cup Y)+\rho_{M}(H \cap Y)
\end{aligned}
$$

Portanto $H, Y \in F$ formam um par modular em $M$, donde $X=H \cap Y \in \bar{\xi}$, uma contradição. Logo $\xi_{1}-\xi=\emptyset$, isto é, $\xi_{1}=\xi$.

Se $S$ é uma subclasse linear de $M$ e $\zeta$ é o filtro modular dado pelo Lema 1.1., diremos que $Z$ é o flltro modular associado a $S$, ou que $S$ é a subclasse linear assaciada a?.

Passemos a descrever uma construção sequencial de subclasses lineares que, embora aimples e natural, será útil na próxima seção. A idéia é partir de uma subfamflia de $K e$, a cada passo, incluir os hiperplanos que formam triplas geradoras com pares de fechados do passo anterior. Formalizando, seja $A \in X$. Chamaremos de sequência geradora de $A$ em $M$ a sequência de subfamilias de $X$, $A=A_{0} \subseteq A_{1} \subseteq A_{2} \subseteq \ldots$ onde para cada $i=1,2, \ldots, Z \in A_{i}$ se e somente se $Z \in A_{i-1}$ ou existem $X, Y \in A_{i-1}$ distintos tais que $X, Y, Z$ formam uma tripla geradora. 
Proposição 1.s. Seja $A \subseteq K$ e $\left\{A_{i}\right\}_{i=0}^{\infty}$ sua sequência geradora. Então $\bigcup_{i=0}^{\infty} A_{i}$ é a menor subclasse linear de $M$ contendo $A$.

Prova: Que $\bigcup_{i=0}^{\infty} A_{i}$ é uma subclasse linear que contém $A$ é evidente. Dada $S$ uma subclasse linear contendo $A$ uma indução trivial mostra que $A_{i} \subseteq S$ para todo $i=0,1, \ldots$ donde obtemos a minimalidade requerida.

Observe que a intersecção de duas subclasses lineares é também uma subclasse linear. Portanto, se $A \subseteq K$ e $\left\{A_{i}\right\}_{i=0}^{\infty}$ é sua sequência geradora então

$$
\bigcup_{i=0}^{\infty} A_{i}=\bigcap\left\{\mathcal{S}^{\prime} \text { subclasse linear de } M: A \subseteq S^{\prime}\right\}
$$

\section{PARES DE SUBCLASSES LINEARES COMPATVEIS}

Diremos que duas subclasses lineares são compatíveis se seus filtros modulares associados também o forem.

Seja $S$ uma subclasse linear de $M$ e $N$ uma extensão de $M$. A subclasse linear

$$
\bigcap\left\{S^{\prime} \text { subclasse linear de } N:\left\{\bar{X}^{N}: \mathbb{X} \in S\right\} \subseteq S^{\prime}\right\}
$$

de $N^{\prime}$ será denotada por $\left[\left.S\right|_{N}\right.$, fecho de $S$ em $N$, e $\left\{\bar{X}^{N}: X \in S\right\}=S_{0}, S_{1}, \ldots$ sua sequência geradora.

Adaptemos a caracterização de pares de filtros modulares compativeis dada pela Proposição 3.4. do Capítulo II ao contexto de subclasses lineares.

Proposição 2.1. Sejam $S_{1}, S_{2}$ subclasses lineares de $M$ e considere as seguintes extensöes pontuais

$$
M(E) \stackrel{\xi_{1}}{\longrightarrow} N_{1}\left(E \cup p_{1}\right) \text { e } M(E) \stackrel{\xi_{2}}{\longrightarrow} N_{2}\left(E \cup p_{2}\right)
$$

onde $\xi_{1}, \xi_{2}$ são os filtros modulares associados a $S_{1}, S_{2}$ respectivamente. Considere também a subclasse linear $\left[\left.S_{1}\right|_{N_{2}}\right.$ de $N_{3}$ com sequência geradora $S_{1}^{0}, S_{1}^{1}, \ldots$ 
e a subclasse linear $\left[S_{2}\right]_{N_{1}}$ de $N_{1}$ com sequência geradora $S_{2}^{0}, S_{2}^{1}, \ldots$ Então são equivalentes:
i. $S_{1}, S_{2}$ são compativeis
ii. $S_{1}=\left\{X \in \mathcal{F}_{M}: \bar{X}^{N_{3}} \in\left[\left.S_{1}\right|_{N_{3}}\right\}=\left\{X \in \mathcal{F}_{M}: \bar{X}^{N_{3}} \in \mathcal{S}_{1}^{i}, i=0,1, \ldots\right\}\right.$
i⿱宀⿰夕㔾. $S_{2}=\left\{X \in \mathcal{F}_{M}: \bar{X}^{N_{1}} \in\left[\left.S_{2}\right|_{N_{1}}\right\}=\left\{X \in \mathcal{F}_{M}: \bar{X}^{N_{1}} \in \mathcal{S}_{3}^{i}, i=0,1, \ldots\right\}\right.$

Ao estudarmos a compatibilidade de duas subclasses lineares, o Corolário 4.4. do Caṕtulo II nos permite restringir a atenção aos pares de subclasses lineares comparáveis por inclusão, como já observado no início deste capítulo. Vamos assumir daquil até o fnal da seção que estamos nas hipbteses da. Proposição 2.1. e que $S_{2} \subseteq S_{1}$.

Analisemos a subclasse linear $\left[\left.S_{2}\right|_{N_{1}}\right.$ com sequência geradora $\left\{\bar{W}^{N_{1}}: W \in\right.$ $\left.S_{2}\right\}=S_{2}^{0}, S_{2}^{1}, \ldots$ Note que como $S_{2} \subseteq S_{1}, S_{2}^{0}=\left\{W \cup p_{1}: W \in S_{2}\right\}$. Para fixar - idéias, tentemos encontrar propriedades dos $W \in S_{2}^{1}-S_{2}^{0}$.

Por definição, existem $X, Y \in S_{2}$ tais que $X \cup p_{1}, Y \cup p_{1}, W$ formam uma tripla geradora de $N_{1}$. Existe então $Z$ tal que $W=Z \cup p_{1}$ e $X \cap Y \subseteq Z$. É fácil ver que $Z \notin X$, pois caso contrário $Z$ pertenceria a $S_{2}$, o que implicaria em $W \in S_{2}^{0}$, uma contradição. Logo $Z$ é uma colinha de $M$ que cobre $X \cap Y$ e $\mathbb{Z} \notin \mathcal{F}_{1}$. Propriedades semelhantes são verificadas também quando $W \in S_{2}^{i}-S_{2}^{i-1}$ para $i=2,3, \ldots$. Tentaremos organizar essas últimas idéias na próxima definição e no próximo lema.

Definimos a seguinte sequência

$$
\emptyset=B_{0}\left(S_{1}, S_{2}\right) \subseteq B_{1}\left(S_{1}, S_{2}\right) \subseteq B_{2}\left(S_{1}, S_{2}\right) \subseteq \ldots
$$

de subfamílias do conjunto $C o l_{1}$ de colinhas de $M$ não cobertas por elementos de $S_{1}$, onde para todo $i=1,2, \ldots, Z \in B_{i}\left(S_{1}, S_{2}\right)-B_{i-1}\left(S_{1}, S_{2}\right)$ se e somente se $Z \in C o l_{1}$ e existem $X, Y$ distintos pertencentes a $S_{2} \cup B_{i-1}\left(S_{1}, S_{2}\right)$ tais que $Z$ cobre $X \cap Y$. Denotaremos por $\left(\$_{1}, \$_{2}\right)$ a união $\bigcup_{i=0}^{\infty} \hat{b}_{i}\left(\$_{1}, \$_{2}\right)$.

Lema 2.2. Para cada $i=0,1,2, \ldots$

$$
\left\{Z \cup p_{1}: Z \in S_{2} \cup B_{i}\left(S_{1}, S_{2}\right)\right\} \subseteq S_{2}^{i}
$$

Prova: Por indução gobre $i$. Se $i=0$ então $\hat{B}_{0}\left(S_{1}, S_{2}\right)=\emptyset \mathrm{e}$

$$
S_{2}^{0}=\left\{\bar{Z}^{N_{1}}: Z \in S_{2}\right\}=\left\{Z \cup p_{1}: Z \in S_{2}\right\}
$$


Suponha que $i>0$ e seja $Z \in S_{2} \cup B_{i}\left(S_{1}, S_{2}\right)$. Se $Z \in S_{2} \cup B_{i-1}\left(S_{1}, S_{2}\right)$ então, por hipótese de indução, $Z \cup p_{1} \in S_{2}^{i-1} \subseteq S_{2}^{i}$. De outro lado, seja $Z \notin$ $S_{2} \cup B_{i-1}\left(S_{1}, S_{2}\right)$. Então $Z \in B_{i}\left(S_{1}, S_{2}\right)-B_{i-1}\left(S_{1}, S_{2}\right)$. Pela definição dos $B_{i}$ 's temos que $Z \in C$ ol 1 existem $X, Y \in S_{2} \cup B_{i-1}\left(S_{1}, S_{2}\right)$ tais que $Z$ cobre $X \cap Y$. Logo $Z \cup p_{1}, X \cup p_{1}, Y \cup p_{1}$ formam uma tripla geradora em $N_{1}$. Novamente usando a hipótese de indução obtemos $X \cup p_{1}, Y \cup p_{1} \in S_{2}^{i-1}$ donde $Z \cup p_{1} \in S_{2}^{i}$. Portanto,

$$
\left\{Z \cup p_{1}: Z \in S_{2} \cup B_{i}\left(S_{1}, S_{2}\right)\right\} \subseteq S_{2}^{i}
$$

Se na Proposição acima valer a igualdade, teremos uma condição necessária e suficiente para que $S_{1}, S_{2}$ sejam compatíveis. Esse fato ficará claro na prova do próximo Teorema onde apresentamos uma caracteriação equivalente à essa igualdade que tem a vantagem de detectar exatamente a situação que bloqueia a compatibilidade.

Teorem 2.8 Sejam $S_{1}, S_{2}$ subclasses lineares de $M$ tajs que $S_{2} \subseteq S_{1}$ e seja $\xi_{1} 0$ fllo modular associado a $S_{1}$. Então são equivalentes:

i. $S_{1}, S_{2}$ são compativeis

ii. Não existem $X, Y \in S_{2} \cup B\left(S_{1}, S_{2}\right)$ e $W \in S_{1}-S_{2}$ tajs que $X \cap Y$ é coplano de $M, X \cap Y \notin \tilde{F}_{1}$ e $X \cap Y \subseteq W$

Prova: No que se segue $M(E) \stackrel{\xi_{1}}{\longrightarrow} N_{1}\left(E \cup p_{1}\right)$ e $\left\{S_{2}^{i}\right\}_{i=0}^{\infty}$ as sequência geradora da subclasse linear $\left[S_{2}\right]_{N_{1}}$.

Inicialnente mostremos que $i . \Rightarrow 8 \%$. Suponhamos por absurdo que $S_{1}, S_{2}$ são compativeis e que existem $X, Y \in S_{2} \cup B\left(S_{1}, S_{2}\right)$ e $W \in S_{1}-S_{2}$ tais que $X \cap Y$ á um coplano de $M, X \cap Y \notin \xi_{1}$ e $X \cap Y \subseteq W$. Existe $i \geq 0$ tal que $X$, $Y \in S_{2} \cup B_{i}\left(S_{1}, S_{2}\right)$, e então pelo Lema anterior, $X \cup p_{1}, Y \cup p_{1} \in S_{2}^{\dot{i}}$. Como $X \cap Y$ é um coplano de $M$ e $X \cap Y \notin \xi_{1}$ temos que $(X \cap Y) \cup p_{1}$ é uma colinha de $N_{1}$. E claro que $W \cup p_{1}$ é um coponto de $N_{1}$ ค $\left(X \cup p_{1}\right) \cap\left(Y \cup p_{1}\right)=(X \cap Y) \cup p_{1} \subseteq W \cup p_{p_{1}}$ donde $W \cup p_{1} \in S_{3}^{i}$. Como $S_{1}, S_{2}$ são compativeis, temos pela Proposição 2.1. que $W \in \mathcal{S}_{3}$, uma contradição.

Verifiquemos agora que ii. $\Rightarrow$ i.. Mostremos por indução sobre i que

$$
S_{2}^{i} \subseteq\left\{Z \cup p_{1}: Z \in S_{2} \cup B_{i}\left(S_{1}, S_{2}\right)\right\}
$$


pois nesse caso, pelo Lema 2.2 , temos na realidade igualdade. Então $\left\{X \in \xi_{M}\right.$ : $\left.\bar{X}^{N_{1}} \in S_{2}^{i}\right\} \subseteq S_{2}, \log 0 S_{2}=\left\{X \in \bar{J}_{M}: \bar{X}^{N_{1}} \in S_{2}^{i}, i=0,1, \ldots\right\}$ e pela Proposição 2.1., $S_{1}, S_{2}$ seriam compativeis. Se $i=0$,

$$
S_{2}^{0}=\left\{Z \cup p_{1}: Z \in S_{2}\right\}=\left\{\mathscr{Z} \cup p_{1}: Z \in S_{2} \cup B_{0}\left(S_{1}, S_{2}\right)\right\}
$$

Suponha então que $i>0$ e seja $W \in S_{2}^{i}$. Se $W \in S_{2}^{i-1}$ então por hipótese de indução

$$
W \in\left\{\mathbb{Z} \cup p_{1}: \mathbb{Z} \in S_{2} \cup B_{i-1}\left(S_{1}, S_{2}\right)\right\} \subseteq\left\{\mathbb{Z} \cup p_{1}: \mathbb{Z} \in S_{2} \cup B_{i}\left(S_{1}, S_{2}\right)\right\}
$$

Tomemos então $W \in S_{2}^{i}-S_{2}^{i-1}$. Pela definição de $S_{2}^{i}$, existem $X, Y \in S_{2}^{i-1}$ tais que $X, Y, W$ formam nma tripla geradora em $N_{1}$. Examinemos os seguintes fechados de $M, X-p_{1}, Y-p_{1} \in W-p_{1}$. Como $X, Y \in S_{2}^{i-1}$, por hipótese de indução temos que

$$
X-p_{1}, Y-p_{1} \in S_{2} \cup B_{i-1}\left(S_{1}, S_{2}\right)
$$

Suponhamos por absurdo que $X-p_{1}, Y-p_{1} \in S_{2}$. Nesse caso, como $X, Y, W$ formam uma tripla geradora em $N_{1}$, temos que $X-p_{1}, Y-p_{1}, W-p_{1}$ formam uma tripla geradora em $M$, donde concluímos que $W-p_{1} \in S_{2}$. Mas então $W={\overline{W-p_{1}}}^{N_{1}} \in S_{2}^{0}$, uma contradição ja que $W \in S_{3}^{i}-S_{2}^{i-1}$ com $i>0$. Podemos entâo supor sem perda de generalidade que $X-p_{1} \in B_{i-1}\left(S_{1}, S_{2}\right)$. Note que $\left(X-p_{1}\right) \cap\left(Y-p_{1}\right)=(X \cap Y)-p_{1}$ não é um elemento de $f_{1}$ pois caso contrário $X-p_{1}$ seria coberto por um elemento de $S_{1}$ e portanto não poderia pertencer a $B_{i-1}\left(S_{1}, S_{2}\right)$. Resumindo,

$$
\Psi_{1} \not \supset\left(X-p_{1}\right) \cap\left(Y-p_{1}\right) \text { é um coplano de } M
$$

Observe também que

$$
\left(X-p_{1}\right) \cap\left(Y-p_{1}\right) \subseteq W-p_{1}
$$

Se $W-p_{1}$ é coponto de $M$ então $W-p_{1} \in S_{1}-S_{2}$ e combinando com $(I),(I I)$, (III) contradizemos ii.. Chegamos à mesma contradição se $W-p_{1}$ é coberto por algum elemento de $S_{1}$. Portanto $W-p_{1}$ é uma colinha de $M$ não coberta por elementos de $S_{1}$. Combinando com $(I)$ temos que $W-p_{1} \in B_{i}\left(S_{1}, S_{2}\right)$, donde $W \in\left\{Z \cup p_{1}: Z \in \mathcal{S}_{2} \cup B_{i}\left(S_{1}, S_{2}\right)\right\}$. 

Capitulo IV

\section{Representabilidade de Quocientes}

Cheung e Crapo em [Ch C], introduziram o conceito de feixe de quocientes que descrevem completamente as extensões de um matróide. Veremos neste capítulo que esse conceito nos fornece uma nova direção no estudo da compatibilidade: a teoria da representabilidade de quocientes.

A primeira seção é dedicada à bijeção entre os quocientes elementares e os filtros modulares.

O objetivo da Seção 2 é estabelecer condições sobre uma família de quo. cientes para que ela determine uma única extensão de um dado matróide.

A Seção 3 é bastante técnica e serve principalmente como preparação para a seção seguinte. Nela examinamos uma construção de uma sequência de quocientes elementares muito utiliada na teoria das extensōes.

A última seção trata do problema da representabilidade de quocientes, mais especificamente de pares de yuocientes elementares. 


\section{A RELAÇA ENTRE QUOCIENTES E MILTROS MODUIARES}

Os quocientes elementares têm grande importância neste capitulo. Isso se deve ao fato de que os filtros modulares não triviais de um matróide $M$ estão em correspondência biunf́roca com os quocientes elementares de $M$, a exemplo do que ocorre com as subclasses lineares. Os três lemas a seguir comprovam essa afirmação. 0 primeiro deles, de caráter mais geral, estabelece uma função que associa um filtro modular de um matróide fixado a cada quociente do mesmo. Infelizmente essa função não é uma bijeção. No entanto, ao restring $1 \cdot$ la a as quocientes elementares do matróide, o segundo e o terceiro lema garantem, respectivamente, a sobrejetividade e a injetividade. Nesse ponto aconselhamos o leitor a rever as definições e resultados da Seção 4 das Preliminares.

Lem 1.1. Sejam $M_{1}, M_{2}$ matróides sobre $E$ tajs que $M_{2}$ é quociente de $M_{1}$. Então o conjunto defnido abaixo

$$
M\left(M_{1} \rightarrow M_{2}\right)=\left\{X \in \bar{J}_{M_{2}}: n l(X)=\operatorname{gr}\left(M_{1} \rightarrow M_{2}\right)\right\}
$$

é um filtro modular de $M_{1}$. Além disso $M\left(M_{1} \rightarrow M_{2}\right)$ é o major flitro modular de $M_{1}$ contido em $\bar{s}_{M_{2}}$.

Prova: Mostremos inicialmente que $M\left(M_{1} \rightarrow M_{2}\right)$ é realmente um filtro modular de $M_{1}$. Que $M\left(M_{1} \rightarrow M_{2}\right)$ satisfaz a propriedade (F1) de filtros modulares segue do item ïi. da Proposição (Morfismo Forte 1) das Preliminares. A propriedade (F2) é decorrência de (F1) combinado com o ítem iv. da Proposição (Mortismo Forte 2) também das Preliminares.

Prosseguindo, suponba que $M\left(M_{1} \rightarrow M_{2}\right)$ não seja um subconjunto de $\Psi_{M_{2}}$. Isso equivale a direr que existe $X \in M\left(M_{1} \rightarrow M_{2}\right)$ tal que $\bar{X}^{M_{2}}-X \neq \emptyset$. Tome então $x \in \bar{X}^{M_{2}}-X$. Nesse caso, $\overline{X \cup x}{ }^{M M_{2}} \in M\left(M_{1} \rightarrow M_{2}\right)$, o que nos condus ao seguinte absurdo

$$
\begin{aligned}
\operatorname{gr}\left(M_{1} \rightarrow M_{2}\right) & =n l(X \cup x) \\
& =\rho_{M_{1}}(X \cup x)-\rho_{M_{2}}(X \cup x) \\
& =\rho M_{2}(X)+1-\rho_{M_{2}}(X)=\operatorname{gr}\left(M_{1} \rightarrow M_{2}\right)+1
\end{aligned}
$$

Portanto, $M\left(M_{1} \rightarrow M_{2}\right) \subseteq M_{2}$.

Resta ainda provarmos que $M\left(M_{1} \rightarrow M_{3}\right)$ é o maior filtro modular de $M_{1}$ totalmente contido em $\xi_{M_{2}}$. Suponha que $I$ seja um filtro modular de $M_{1}$ que 
contém propriamente o filtro $M\left(M_{1} \rightarrow M_{2}\right)$. Existe então um hiperplano $H$ de $M_{1}$ tal que $H \in \xi-M\left(M_{1} \rightarrow M_{2}\right)$. Com isso obtemos a seguinte desigualdade

$$
\begin{aligned}
\left(\rho M_{1}-1\right)-\rho_{M_{2}}(H) & =\rho_{M_{1}}(H)-\rho_{M_{2}}(H)=n l(H) \\
& <g r\left(M_{1} \rightarrow M_{2}\right)
\end{aligned}
$$

Assim, $\rho_{M_{3}}(X)=\rho M_{2}$, o que implicaria que $H \notin I_{M_{2}}$, pois caso contrário teŕamos $H=E \in F \cap M\left(M_{1} \rightarrow M_{2}\right)$, um absurdo. Logo $q \Psi_{M_{2}}$.

Lema 1.2. Sejam $\zeta$ um filtro modular de $M$ não trivial e a extensão pontual $M(E) \stackrel{F}{\longrightarrow} N(E \cup p)$. Enż̃o $N / p$ é quociente elementar de $M$ e $\xi=M(M \rightarrow$ $N / p)$.

Prova: Como $N$ é a extensão pontual de $M$ por $q$ abemos que

$$
\begin{aligned}
\xi_{N}=\{X \cup p: X \in \xi\} \cup\left(\xi_{M}-\xi\right) \cup\left\{X \cup p: X \in I_{M}-\xi\right. \\
X \text { não é coberto por elemento de } \xi\}
\end{aligned}
$$

Logo, lembrando as propriedades da operação de contração em matróides, $\rho N / \rho=$ $\rho M-1$ ja que $\neq \bar{F}_{M}$ e o conjunto dos fechados de $N / p$ é dado por

$$
\tilde{I}_{N / p}=\mathcal{F} \cup\left\{X \in \mathcal{F}_{M}-\mathcal{F}: X \text { não é coberto por elemento de }\right\}
$$

Assim é fácil ver que $N / p$ é quociente elementar de $M$.

Mostremos que $=M(M \rightarrow N / p)$. Para isso basta verificarmos que $M(M \rightarrow N / p) \subseteq$ pois nesse caso a igualdade segue da maximalidade de $M(M \rightarrow$ $N / p)$ em relação aos filtros modulares de $M$ contidos em $\xi_{N / p}$. Seja $X \in M(M \rightarrow$ $N / p)$, isto é, $n l(X)=\operatorname{gr}(M \rightarrow N / p)$. Mas

$$
\begin{gathered}
n l(X)=\rho_{M}(X)-\rho_{N / p}(X)=\rho_{M}(X)-\rho_{N}(X \cup p)+1 \\
g r(M \rightarrow N / p)=\rho M-\rho N / p=\rho M-\rho N+1=1
\end{gathered}
$$

Logo, $\rho_{M}(X)=\rho_{N}(X \cup p)$, o que implica que $X \in \mathcal{F}$.

Lem 1.8. Seja $Q$ um quociente elementar de $M$ e considere a extensão pontual $M(E) \stackrel{\xi}{\longrightarrow} N(E \cup p)$ onde $\xi=\mathcal{M}(M \rightarrow Q)$. Então $Q=N / p$.

Prova: Observe que $\zeta$ é não trivial pois $Q$ é quociente elementar de $M$. Vamos mostrar que $\xi_{N / p}=\xi_{Q}$. Lembremos que

$$
\xi_{N / p}=\xi \cup\left\{X \in \xi_{M}-\xi: X \text { não é coberto por elemento de } \xi\right\}
$$


Seja $X \in \xi_{Q}$. Suponha por absurdo que $X \notin \xi_{N / p}$, isto $e$, que $X \notin \xi$ e existe $x \in E$ tal que $\overline{X \cup x}^{M} \in \mathcal{f}$. Com isso temos $n l(X)=0, n l(X \cup x)=$ 1 e $\rho_{M}(X \cup x)=\rho_{M}(X)+1$, donde se conclui que $\rho_{Q}(X \cup x)=\rho_{Q}(X)$, ou equivalentemente, $x \in \bar{X}^{Q}=X=\bar{X}^{M}$, uma contradição. Assim $\mathcal{F}_{Q} \subseteq \mathcal{F}_{N / p}$.

A inclusão contrária também é verdadeira. De fato, $\bar{Z} \subseteq \bar{F}_{Q}$, como já foi observado anteriormente, e suponha que $X \in \bar{\zeta}_{M}-I_{\text {não }}$ é coberto por elemento de $\xi$. Se $x \in \bar{X}^{Q}$, como $X, X \cup x \notin \xi$, então

$$
\rho_{M}(X \cup x)=\rho_{Q}(X \cup x)=\rho_{Q}(X)=\rho_{M}(X)
$$

e portanto $x \in \bar{X}^{M}=X$, o que mostra que $X \in \mathcal{F}_{Q}$. Conclufmos finalmente que $Q=N / p$.

\section{FEIXES DE QUOCIRNTES}

Seja $N$ uma extensão de $M$ sobre $E \cup P$. $O$ feixe de quacierbtes de $M$ determinado por $N$ é definido como sendo a famflia

$$
\{(N / Y)(E): Y \text { é fechado de } N(P)\}
$$

Denotaremos $(N / Y)(E)$ por $Q_{Y}$. Vejamos algumas propriedades dessa familia.

Proposição 2.1. Seja $N$ uma extensão de $M$ sobre $E \cup P$ e denote $N(P)$ por $R$. Então o feixe de quocientes de $M$ determinado por $N$ tem as seguintes propriedades:

(Q1) $Q_{0}=M$

(Q2) Se $X, Y \in \mathcal{F}_{R}$ e $Y$ cobre $X$ então $Q_{Y}$ é quociente de $Q_{X}$ e $g r\left(Q_{X} \rightarrow\right.$ $\left.Q_{Y}\right) \leq 1$

(Q3) Sejam $X, Y \in \xi_{R}$ tais que $X \subseteq Y$. Definimos

$$
M(X, Y)=\left\{Z \in \xi_{Q_{X}}: n l_{Q_{X} \rightarrow Q_{Y}}(Z)=\rho_{R}(Y)-\rho_{R}(X)\right\}
$$

Temos então que $M(X, Y)$ é um filtro modular de $Q X$ e, além disso, se $X$, $Y \in \mathcal{F}_{R}$ são tais que $X, Y$ cobrem $X \cap Y$ então

$$
\mathcal{M}(X \cap Y, X) \cap \mathcal{M}(X \cap Y, Y)=M\left(X \cap Y, \overline{X \cup Y}^{R}\right)
$$


Prova: (Q1) é trivial. Tome $X, Y \in \xi_{R}$ tal que $Y$ cobre $X$. Então $X, Y$ satisfazem (Q2), isto é, $Q_{Y}$ é quociente elementar de $Q_{X}$. De fato, seja $Z \in Q_{Y}$. Segue das definições de contração e restrição que existe $Z^{\prime} \in Z_{N}$ tal que $Y \subseteq Z^{\prime}$ e $Z=\left(Z^{\prime}-Y\right) \cap E$. Uma vez que $X \subseteq Y \subseteq P$, resulta que $Z=\left(Z^{\prime}-X\right) \cap E$, e porłanto $Z \in Q_{X}$. Alén disso é fácil ver que $g r\left(Q_{X} \rightarrow Q_{Y}\right) \leq \rho_{R}(Y)-\rho_{R}(X)=1$.

Verifiquemos (Q3). Tome inicialmente $X, Y \in \xi_{R}$ tais que $X \subseteq Y$ e $M(X, Y)$ como definido no enunciado. Decorre facilmente de (Q2) que $Q Y$ é quociente de $Q_{X}$. Aplicando o item äia. da Proposição (Morísmo Forte 1) das Preliminares temos a seguinte desiguald ade para todo $Z \in \mathcal{F}_{q_{x}}$

$$
n l_{Q_{X} \rightarrow Q_{Y}}(Z) \leq \operatorname{gr}\left(Q_{X} \rightarrow Q_{Y}\right) \leq \rho_{R}(Y)-\rho_{R}(X)
$$

Logo, se $\operatorname{gr}\left(Q_{X} \rightarrow Q_{Y}\right)=\rho_{R}(Y)-\rho_{R}(X)$ então $\mathcal{M}(X, Y)=\mathcal{M}\left(Q_{X} \rightarrow Q_{Y}\right)$, caso contrário $M(X, Y)=\emptyset$, o que prova que $M(X, Y)$ é realmente um filtro modular de $Q x$.

Considere agora $X, Y \in \mathcal{F}_{R}$ tais que $X, Y$ cobrem $X \cap Y$ e provemos que $\mathcal{M}(X \cap Y, X) \cap M(X \cap Y, Y)=M\left(X \cap Y, \overline{X \cup Y}^{R}\right)$ verificando as duas inclusões possiveis. Tome $\mathbb{Z} \in M(X \cap Y, X) \cap \mathcal{M}(X \cap Y, Y)$. Por definição, $\rho_{Q X \cap Y}(Z)=$ $\rho_{Q_{X}}(Z)+1=\rho_{Q_{Y}}(Z)+1$. IBso implica que $\rho_{N}(Z \cup(X \cap Y))=\rho_{N}(Z \cup X)=$ $\rho_{N}(Z \cup Y)$ lembrando que os elementos do feixe são contraçōes seguidas de restriçöes do matroide $N$. Mas então

$$
\begin{aligned}
\rho_{N}\left(Z \cup \overline{X \cup Y}^{R}\right) & \geq \rho_{N}(Z \cup(X \cap Y)) \\
& =\rho_{N}(Z \cup X)+\rho_{N}(Z \cup Y)-\rho_{N}(Z \cup(X \cap Y)) \\
& \geq \rho_{N}\left(Z \cup \overline{X \cup Y^{R}}\right)
\end{aligned}
$$

e portanto $\rho_{N}\left(Z \cup \overline{X \cup Y^{R}}\right)=\rho_{N}(Z \cup(X \cap Y))$. Usamos esse fato para concluir que

$$
\begin{aligned}
\rho_{Q_{\overline{X \cup Y}}}(Z) & =\rho_{N}\left(Z \cup \overline{X \cup Y^{R}}\right)-\rho_{N}\left(\overline{X \cup Y^{R}}\right) \\
& =\rho_{N}(Z \cup(X \cap Y))-\rho_{N}(X \cap Y)-2 \\
& =\rho_{Q_{X \cap Y}}(Z)-Z
\end{aligned}
$$

Logo $Z \in M\left(X \cap Y, \overline{X \cup Y}^{R}\right)$. De outro lado, fixe agora $Z \in M\left(X \cap Y, \overline{X \cup Y}{ }^{R}\right)$. Assim $\rho_{Q \times \text { N }^{\prime}}(Z)=\rho_{Q} \overline{\overline{X \cup Y}}^{R}(Z)+2$, ou de outra forma, $\rho_{N}\left(Z \cup{\overline{X \cup Y^{R}}}^{R}\right)=\rho_{N}(Z \cup$ $(X \cap Y))$. Mas $\rho_{N}(Z \cup(X \cap Y)) \leq \rho_{N}(Z \cup X) \leq \rho_{N}\left(Z \cup \overline{X \cup Y^{R}}\right)$ e então $\rho_{N}(Z \cup X)=\rho_{N}(Z \cup(X \cap Y))$, portanto

$$
\begin{aligned}
p_{Q_{X}}(Z) & =p_{N}(Z \cup X)-p_{N}(X) \\
& =p_{N}(Z \cup(X \cap Y))-p_{N}(X \cap Y)-1 \\
& =p_{Q_{X} \cap}(Z)-1
\end{aligned}
$$


donde se conclui que $Z \in M(X \cap Y, X)$. Analogamente mostra-se que $Z \in M(X \cap$ $Y, Y)$.

Vale ressaltar que em $\left(Q_{2}\right), \operatorname{gr}\left(Q_{X} \rightarrow Q_{Y}\right)=0$ se e somente se $Q_{X}=Q_{Y}$, como mostra o resultado seguinte.

Lema 2.2. Sejam $M_{1}, M_{2}$ matróides sobre $E$ tais que $M_{2}$ é quociente de $M_{1}$. Então $\rho M_{1}=\rho M_{2}$ se e somente se $M_{1}=M_{2}$.

Prova: A necessidade é evidente. Para verif́carmos a suficiência fixemos $X \subseteq E$. Como $M_{2}$ é quociente de $M_{1}$ é claro que $\rho_{M_{2}}(X) \leq \rho_{M_{2}}(X)$, e aplicando o ítem iị. da Proposição (Morfismo Forte 1) das Preliminares, temos em particular que $\rho M_{2}-\rho_{M_{2}}(X) \leq \rho M_{1}-\rho_{M_{1}}(X)$. Mas por hipótese, $\rho M_{1}=\rho M_{2}, \log \circ \rho M_{2}(X) \geq$ $\rho_{M_{1}}(X)$. Portanto, $\rho_{M_{1}}(X)=\rho_{M_{2}}(X)$, donde resulta a igualdade $M_{1}=M_{2}$.

O item (Q3) vale num contexto mais geral:

Proposição 2.s. Se $M_{1}, M_{2}$ e $M_{3}$ são matróides sobre $\bar{E}$ tais que $M_{3}$ é quociente de $M_{2}$ e $M_{2}$ é quociente de $M_{1}$ então

$$
M\left(M_{1} \rightarrow M_{3}\right)=M\left(M_{1} \rightarrow M_{2}\right) \cap M\left(M_{2} \rightarrow M_{3}\right)
$$

Prova: Se $X \in M\left(M_{1} \rightarrow M_{2}\right) \cap M\left(M_{2} \rightarrow M_{3}\right)$ então $X \in M\left(M_{1} \rightarrow M_{3}\right)$. De fato

$$
\begin{aligned}
n l_{M_{2} \rightarrow M_{3}}(X) & =\rho_{M_{2}}(X)-\rho_{M_{8}}(X) \\
& =\left(\rho_{M_{1}}(X)-\rho_{M_{2}}(X)\right)+\left(\rho_{M_{2}}(X)-\rho_{M_{8}}(X)\right) \\
& =g r\left(M_{1} \rightarrow M_{2}\right)+g r\left(M_{2} \rightarrow M_{3}\right) \\
& =\operatorname{gr}\left(M_{1} \rightarrow M_{3}\right)
\end{aligned}
$$

De outro lado, seja $X \in M\left(M_{1} \rightarrow M_{3}\right)$, isto é, $n l_{M_{2} \rightarrow M_{8}}(X)=\operatorname{gr}\left(M_{1} \rightarrow\right.$ $\left.M_{3}\right)$. Assin, $n l_{M_{1} \rightarrow M_{3}}(X)+n l_{M_{2} \rightarrow M_{s}}(X)=\operatorname{gr}\left(M_{1} \rightarrow M_{2}\right)+\operatorname{gr}\left(M_{3} \rightarrow M_{3}\right)$, mas essa igualdade ó é possivel se $n l_{M_{1} \rightarrow M_{2}}(X)=g r\left(M_{1} \rightarrow M_{3}\right)$ e $n l_{M_{2} \rightarrow M_{8}}(X)=$ $\operatorname{gr}\left(M_{2} \rightarrow M_{3}\right)$. Logo $X \in M\left(M_{1} \rightarrow M_{2}\right) \cap M\left(M_{2} \rightarrow M_{3}\right)$. 
Teorem 2.4. Sejam $R$ um matróide sobre $P$ e $\left\{Q(Y): Y \in \xi_{R}\right\}$ uma fam llia de quocientes de $M$ satisfasendo as propriedades (Q1), (Q2) e (Q3) da Proposição 2.1. Entz̃o existe uma única extensão $N$ de $M$ sobre $E \cup P$ tal que $N(P)=R$ e $Q(Y)=Q_{Y}=(N / Y)(E)$ para todo $Y \in \mathcal{I}_{R}$.

Prova: Definimos a função $\rho_{N}$ sobre o conjunto das partes de $E \cup P$ da seguinte maneira

para todo $X \subseteq E \in Y \subseteq P$,

$$
\rho_{N}(X \cup Y)=\rho_{Q\left(\bar{Y}^{R}\right)}(X)+\rho_{R}(Y)
$$

Mostremos que $\rho_{N}$ satisfas os axiomas de posto (R2) e (R3) já que o axioma (R1) é consequência imediata da definição de $\rho_{N}$. Para não carregar a notação, denotaremos $\left.\rho_{Q(\bar{Y}}{ }^{R}\right)$ por $\rho_{Y}$ para cada $Y \subseteq P$.

(R2) Sejam $X \subseteq E, Y \subseteq P$ e $w \in E \cup P$. Dividindo em casos:

Caso 1: $w \in E$

Como py satisfag (R2) então

$$
\rho_{Y}(X) \leq \rho_{Y}(X \cup \rightsquigarrow) \leq \rho_{Y}(X)+1
$$

Desse fato segue que

$$
\rho_{N}(X \cup Y) \leq \rho_{N}(X \cup Y \cup w) \leq \rho_{N}(X \cup Y)+1
$$

Caso 2: $w \in P$

Se $\in \in \bar{Y}^{R}$ então $\rho_{Y}=\rho_{Y} \cup$ e $\rho_{R}(Y)=\rho_{R}(Y \cup w)$. Assim

$$
\rho_{N}(X \cup Y)=\rho_{N}(X \cup Y \cup w)<\rho_{N}(X \cup Y)+1
$$

Por outro lado, se $w \& \bar{Y}^{R}$ decorre de $(Q 2)$ que $Q\left(\overline{\left.Y \cup w^{R}\right)}\right.$ é quociente de $Q\left(\bar{Y}^{R}\right)$ e $\operatorname{gr}\left(Q\left(\bar{Y}^{R}\right) \rightarrow Q(\overline{Y \cup w})\right) \leq 1$, logo $\rho_{Y}(X)-\rho_{Y \cup_{w}}(X) \leq 1$. Temos então que

$$
\begin{aligned}
\rho_{N}(X \cup Y \cup w) & =\rho_{Y} \cup(X)+\rho_{R}(Y \cup w) \\
& =\rho_{Y}(X)+\rho_{R}(Y)+(1 \text { ou } 0) \\
& =\rho_{N}(X \cup Y)+(1 \text { ou } 0)
\end{aligned}
$$

(R3) Sejam $X \subseteq E, Y \subseteq P$ e w, $z \in E \cup P$ tais que $\rho_{N}(X \cup Y \cup w)=\rho_{N}(X \cup$ $Y \cup z)=\rho_{N}(X \cup Y)$. Temos então três casos a considerar: 
Caso 1: $w, z \in E$

Decorre da hipótese e da definição de $\rho N$ que

$$
\rho_{Y}(X \cup w)=\rho_{Y}(X \cup z)=\rho_{Y}(X)
$$

Novamente observando que $\rho Y$ é uma função posto concluímos que $\rho_{Y}(X \cup w \cup z)=\rho_{Y}(X)$. Logo

$$
\begin{aligned}
\rho_{N}(X \cup Y \cup w \cup z) & =\rho_{Y}(X \cup w \cup z)+\rho_{R}(Y) \\
& =\rho_{Y}(X)+\rho_{R}(Y) \\
& =\rho_{N}(X \cup Y)
\end{aligned}
$$

Caso 2: $w \in E$ e $z \in P$

$\mathrm{Da}$ igualdade $p_{N}(X \cup Y \cup w)=p_{N}(X \cup Y)$ segue que está no fecho de $X$ em $Q\left(\bar{Y}^{R}\right)$ que será denotado por $\bar{X}^{Y}$. Se $z \in \bar{Y}^{N}$ então

$$
\begin{aligned}
\rho_{N}(X \cup Y \cup \cup \cup z) & =\rho_{Y} \cup(X \cup z)+\rho_{R}(Y \cup z) \\
& =\rho_{Y}(X)+\rho_{R}(Y) \\
& =\rho_{N}(X \cup Y)
\end{aligned}
$$

Se z $\notin \bar{Y}^{N}$ então decorre de $(Q 2)$ que $Q(\overline{Y \cup z})$ é quociente de $Q\left(\bar{Y}^{R}\right)$. Do item ï. da Proposição (Morfismo Forte 1) das Preliminares, $\bar{X}^{Y} \subseteq \bar{X}^{Y \cup z}, \log 0 w \in \bar{X}^{Y \cup z}$. Asim

$$
\begin{aligned}
\rho_{N}(X \cup Y \cup w \cup z) & =\rho_{Y} \cup z \\
& =\rho_{Y} \cup(X)+\rho_{R}(Y \cup z)+\rho_{R}(Y \cup z) \\
& =\rho_{N}(X \cup Y \cup z) \\
& =\rho_{N}(X \cup Y)
\end{aligned}
$$

Caso 3: $w, z \in P$

Se $w \in \bar{Y}^{R}$ ou $w \in \overline{Y \cup z}$ então

$$
\begin{aligned}
\rho_{N}(X \cup Y \cup w \cup z) & =\rho_{Y \cup \cup \cup z}(X)+\rho_{R}(Y \cup w \cup z) \\
& =\rho_{Y \cup Z}(X)+\rho_{R}(Y \cup z) \\
& =\rho_{N}(X \cup Y \cup z) \\
& =\rho_{N}(X \cup Y)
\end{aligned}
$$


Fato semelhante ocorre se $z \in \bar{Y}^{R}$ ou $z \in \overline{Y \cup z}^{R}$. Suponhamos então que $\{w, z\} \cap \bar{Y}^{\mathbb{R}}=\emptyset$ e $w \notin \overline{Y \cup z} \bar{Y}^{\mathbb{R}}$. Nesse caso, da igualdade $\rho_{N}(X \cup Y)=\rho_{N}(X \cup Y \cup w)=\rho_{N}(X \cup Y \cup z)$ decorre que $\rho_{Y}(X)-$ $\rho_{Y \cup u}(X)=1=\rho_{Y}(X)-\rho_{Y \cup Z}(X)$, isto é, $X \in M\left(\bar{Y}^{R}, \overline{Y \cup w}\right) \cap$ $M\left(\bar{Y}^{R}, \overline{Y \cup z}^{R}\right)$. Por (Q3), $X \in M\left(\bar{Y}^{R}, \overline{Y \cup w \cup z}\right)$, on melhor, $\rho_{Y}(X)-\rho_{Y} \cup_{\text {v }}(X)=2$. Então

$$
\begin{aligned}
\rho_{N}(X \cup Y \cup w \cup z) & =\rho_{Y} \cup \cup z(X)+\rho_{R}(Y \cup w \cup z) \\
& =\rho_{Y}(X)+\rho_{R}(Y) \\
& =\rho_{N}(X \cup Y)
\end{aligned}
$$

Nos três casos, $\rho_{N}(X \cup Y \cup w \cup z)=\rho_{N}(X \cup Y)$.

Seja $N$ o matróide sobre $E \cup P$ determinado pela função posto $\rho N$. A familia $\left\{Q(Y): Y \in f_{R}\right\}$ é o feixe de quocientes de $M$ determinado por $N$. De fato, fxado $Y \in \mathcal{F}_{R}$ temos para todo $X \subseteq E$ que $\rho_{Q(Y)}(X)=\rho_{N}(X \cup Y)-\rho_{R}(Y)$, o que mostra que $Q(Y)=N / Y(E)$. Segue de $(Q 1)$ que $N(E)=M$. A unicidade de $N$ como extensão de $M$ sobre $E \cup P$ tal que $N(P)=R$ e $Q(Y)=N / Y(E)$ para todo $Y \in \xi_{R}$ também é clara.

Seja $R$ um matroide sobre $P$. Uma familia $\left\{Q(Y): Y \in \xi_{R}\right\}$ de quocientes de $M$ que satisfas as propriedades $(Q 1),(Q 2)$ e (Q3) da Proposiç̃o 2.1. será chamada de $R$-feixe de $M$. Ele será estrito se para todo $Y \in \mathcal{F}_{R}, \operatorname{gr}(M \rightarrow Q(Y))=$ $\rho_{R}(Y)$. O Teorema 2.4. mostrou que o8 $R$-feixes determinam completamente as extensões $N$ de $M$ sobre $E \cup P$ tais que $N(P)=R$. Em particular, quando $\rho M=\rho N, \circ$ R-feixe será estrito como mostra o próximo resultado.

Proposição 2.5. Sejam $R$ um matróide sobre $P$ e $Q=\left\{Q(Y): Y \in \xi_{R}\right\}$ um $\boldsymbol{R}$-feixe de $M$. São equivalentes:

i. Q é estrito

ii. Para todos $X, Y \in \mathcal{J}_{R}$ tajs que $X \subseteq Y$ temos que gr $(Q(X) \rightarrow Q(Y))=$ $\rho_{R}(Y)-\rho_{R}(X)$

iii. Para todos $X, Y \in \xi_{R}$ tais que $X \subseteq Y$ bemos que $M(X, Y) \neq 0$

iv. Se $N$ é a extensão de $M$ sobre $E \cup P$ tal que $N(P)=R$ e $Q$ é o feixe de guocientes de $M$ determinado por $N$ entäo $p M=p N$. 
Prova: A equivalência entre 3 i. e ïi. já foi observada anteriormente. Provaremos então que $i i . \Leftrightarrow \dot{s}$. $\Leftrightarrow$ iii..

i. $\Rightarrow$ ii.: Sejam $X, Y \in \xi_{R}$ tais que $X \subseteq Y$. Então, usando $i$.

$$
\begin{aligned}
\operatorname{gr}(Q(X) \rightarrow Q(Y)) & =\rho Q(X)-\rho Q(Y) \\
& =(\rho M-\rho Q(Y))-(\rho M-\rho Q(X)) \\
& =\operatorname{gr}(M \rightarrow Q(Y))-\operatorname{gr}(M \rightarrow Q(X)) \\
& =\rho_{R}(Y)-\rho_{R}(X)
\end{aligned}
$$

ii. $\Rightarrow$ i.: Seja $Y \in \xi_{R}$. Ugando $i i$ e (Q1)

$$
\begin{aligned}
\operatorname{gr}(M \rightarrow Q(Y)) & =\operatorname{gr}(Q(Q) \rightarrow Q(Y)) \\
& =\rho_{R}(Y)-\rho_{R}(\varphi) \\
& =\rho_{R}(Y)
\end{aligned}
$$

i. $\Rightarrow$ iv.: De i., $g r(M \rightarrow Q(P))=\rho_{R}(P)$, isto é, $\rho M-\rho Q(P)=\rho R$. Seja $N$ a extensão de $M$ sobre $E \cup P$ com $N(P)=R$ e $Q$ o feixe de quocientes de $M$ determinado por $N$. Podemos reescrever a igualdade acima da seguinte forma

$$
\rho_{N}(E)-\left(\rho_{N}(E \cup P)-\rho_{N}(P)\right)=\rho R
$$

$\operatorname{Mas} \rho_{N}(P)=\rho R, \log \circ \rho_{N}(E)=\rho_{N}(E \cup P)$, o que equivale a $\rho M=\rho N$.

iv. $\Rightarrow$ i.: Fixemos $Y \in \xi_{R}$. Sabemos que para todo $X \subseteq E, \rho_{N}(Y)=\rho_{N}(X \cup Y)-$ $P Q(Y)(X)$. Em particular,

$$
\begin{aligned}
\rho_{N}(Y)=\rho_{N}(E \cup Y)-\rho Q(Y) & \leq \rho N-\rho Q(Y) \\
& =\rho M-\rho Q(Y) \\
& =\operatorname{gr}(M \rightarrow Q(Y))
\end{aligned}
$$

Por outro lado,

$$
\begin{aligned}
\operatorname{gr}(M \rightarrow Q(Y)) & =\operatorname{gr}(Q(\emptyset) \rightarrow Q(Y)) \\
& \leq \rho_{N}(Y)-\rho_{N}(\emptyset) \\
& =\rho_{N}(Y)
\end{aligned}
$$

Portanto, $\operatorname{gr}(M \rightarrow Q(Y))=p_{N}(Y)$. 


\section{SEQUENCIAS DE REBAIXAMENTOS IVVANTAMENTOS}

A técnica de construção apresentada nessa seção foi inventada por Higgs em [Hi] e tem, a partir de então, se mostrado bastante útil em muitos problemas relacionados com quocientes de matróides.

No que se segue, salvo menção em contrário, $M_{1}$ e $M_{2}$ são matróides sobre $E$ tais que $M_{2}$ é quociente de $M_{1}$ e $M_{1} \neq M_{2}$.

Proprosição 8.1. 0 conjunto

$$
\left\{X \in \zeta_{M_{2}}: n l(X) \neq g r\left(M_{1} \rightarrow M_{2}\right)-1\right\} \cup \mathcal{I}_{M_{2}}
$$

é o conjunto dos fechados de um certo matróide.

Prova: Considere o filtro modular

$$
\xi=M\left(M_{1} \rightarrow M_{2}\right)=\left\{X \in \mathcal{I}_{1}: n l(X)=\operatorname{gr}\left(M_{1} \rightarrow M_{2}\right)\right\}
$$

e a seguinte extensão pontual $M_{1}(\bar{E}) \stackrel{\xi}{\longrightarrow} N(E \cup p)$. Mostremos que $\xi_{N / p}=\xi$. Como

$$
\xi_{N / p}=\xi_{M_{3}} \cup\left\{X \in F_{M_{1}}-\xi: X \text { não é coberto por elemento de } \xi\right\}
$$

basta mostrarmos que para todo $X \in \mathcal{F}_{M_{1}}-\Psi_{1} X$ é coberto por algum elemento de $\bar{z}$ se e somente se $X \notin \bar{F}_{M_{2}}$ e $n l(X)=\operatorname{gr}\left(M_{1} \rightarrow M_{2}\right)-1$.

Suponha inicialmente que $X \in \xi_{M_{1}}-\xi_{\text {e existe }} \mathscr{E} \in \mathbb{E}$ tal que $\bar{\xi} \overline{X \cup x}^{M_{1}}$ cobre $X$. Se $\rho_{M_{2}}(X \cup x)=\rho_{M_{2}}(X)+1$ então

$$
\begin{aligned}
\operatorname{gr}\left(M_{1} \rightarrow M_{2}\right) & =n l(X \cup x) \\
& =\rho_{M_{1}}(X \cup x)-\rho_{M_{2}}(X \cup x) \\
& =\left(\rho_{M_{1}}(X)+1\right)-\left(\rho_{M_{2}}(X)+1\right) \\
& =n l(X)
\end{aligned}
$$

isto é, $X \in \xi$, uma contradição. Logo $\rho_{M_{2}}(X \cup x)=\rho_{M_{2}}(X)$ e assim, $n l(X)=$ $\operatorname{gr}\left(M_{1} \rightarrow M_{3}\right)-1$ e $X \notin \mathcal{F}_{M_{2}}$.

De outro lado, seja $X \in \xi_{M_{1}}-\left(\xi \cup \xi_{M_{2}}\right)$ tal que $n l(X)=\operatorname{gr}\left(M_{1} \rightarrow M_{2}\right)-1$. Como $X \notin \bar{\zeta}_{M_{2},}$ existe $x \in \bar{X}^{M_{3}}-X$ e

$$
\begin{aligned}
n l(X \cup x) & =\rho_{M_{1}}(X \cup x)-\rho_{M_{2}}(X \cup x) \\
& =\left(\rho_{M_{2}}(X)+1\right)-\rho_{M_{2}}(X) \\
& =g r\left(M_{1} \rightarrow M_{2}\right)
\end{aligned}
$$


Portanto, $F \ni \overline{X \cup x}^{M}$ cobre $X$.

Definimos então o rebeizamento de $M_{1}$ em direção a $M_{2}$ como sendo o matróide $D\left(M_{1} \rightarrow M_{2}\right)$ sobre $E$ com o conjunto de fechados igual a

$$
\left\{X \in \bar{\zeta}_{M_{2}}: n l(X) \neq g r\left(M_{1} \rightarrow M_{2}\right)-1\right\} \cup \mathcal{F}_{M_{3}}
$$

Pela prova da Proposição anterior é fácil ver que $D\left(M_{1} \rightarrow M_{2}\right)$ é quociente elementar de $M_{1}$ e que $M_{2}$ é quociente de $D\left(M_{1} \rightarrow M_{2}\right)$.

Cheung em $[\mathrm{Ch}]$ define rebaixamento de $M_{1}$ en direção a $M_{2}$ como sendo o suposto matróide com o conjunto de fechados

$$
\left\{X \in \mathcal{F}_{M_{2}}: n l(X) \neq g r\left(M_{1} \rightarrow M_{2}\right)-1\right\}
$$

Essa definição é incorreta como mostra o próximo exemplo.

Exemplo \$.2. Sejam $E=\{a, b, c, d\}$ \& $M_{1}, M_{2}$ os matróides sobre $E$ dados por

M

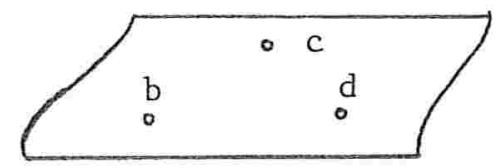

O conjunto

$$
\left\{X \in \mathcal{F}_{M_{1}}: n l(X) \neq g r\left(M_{1} \rightarrow M_{2}\right)-1\right\}=\{\emptyset, a, b, c, d, a c, a d, b c, b d, a b c d\}
$$

não é o conjunto dos fechados de um matroide. De fato, se existisse um matróide com esse conjunto de fechados e função posto $\rho$, teríamos

$$
\begin{gathered}
\rho(a)+\rho(b)=3 \\
\rho(a \vee b)+\rho(a \wedge b)=\rho(a b)+\rho(b)=3
\end{gathered}
$$

que contraria a submodularidade de $\rho$. 
Definimos a seguir a sequência de rebaixamentos estritos de $M_{1}$ até $M_{2}$ como sendo a seguinte sequência de quocientes de $M_{1}$

$$
\begin{aligned}
& D^{0}\left(M_{1} \rightarrow M_{2}\right)=M_{1} \\
& D^{i}\left(M_{1} \rightarrow M_{2}\right)=D\left(D^{i-1}\left(M_{1} \rightarrow M_{2}\right) \rightarrow M_{3}\right), \quad 1 \leq i \leq \operatorname{gr}\left(M_{1} \rightarrow M_{2}\right)
\end{aligned}
$$

Observe que para $i=1,2, \ldots, g r\left(M_{1} \rightarrow M_{2}\right), D^{i}\left(M_{1} \rightarrow M_{2}\right)$ é quociente elementar de $D^{i-1}\left(M_{1} \rightarrow M_{2}\right)$ e se $i \geq \operatorname{gr}\left(M_{1} \rightarrow M_{2}\right), D^{i}\left(M_{1} \rightarrow M_{2}\right)=M_{2}$. Não havendo perigo de ambiguidade, utilisaremos apenas $D^{i}$ para denotar $D^{i}\left(M_{1} \rightarrow M_{2}\right)$, i= $0,1, \ldots, \operatorname{gr}\left(M_{1} \rightarrow M_{2}\right)$.

Uma sequência de rebaixamentos estritos de $M_{1}$ até $M_{2}$ também pode ser obtida "de trás para frente", isto é, obtida recursivamente a partir de $M_{3}=$ $D^{g r}\left(M_{1} \rightarrow M_{2}\right)$.

Proposição 3.s. Sejam $k=\operatorname{gr}\left(M_{1} \rightarrow M_{2}\right)$ e $M_{1}=D^{0}, D^{1}, \ldots, D^{k}=M_{2}$ a sequência de rebaixamentos estritos de $M_{1}$ até $M_{2}$. Temos então que para cada $i=k, k-1, \ldots, 1$

$$
\xi_{D^{i-1}}=\left\{X \in \xi_{M_{1}}: n l_{M_{1} \rightarrow D^{i}}(X)=0\right\} \cup \xi_{D^{i}}
$$

Prova: É necessário apenas verificar o caso $i^{\circ}=k$ pois os demais casos são análogos já que para cada $j=1,2, \ldots, k, D^{j}$ é quociente de $M_{1}, \operatorname{gr}\left(M_{1} \rightarrow D^{j}\right)=j \mathrm{e}$ $D^{0}, D^{1}, \ldots, D^{j}$ é a sequência de rebaixamentos estritos de $M_{1}$ até $D^{j}$. Mostremos então que

$$
\xi_{D^{k-1}}=\left\{x \in \mathcal{F}_{M_{1}}: n l_{M_{1} \rightarrow M_{2}}(X)=0\right\} \cup \xi_{M_{2}}
$$

Por definição,

$$
\xi_{D^{k-1}}=\left\{X \in \xi_{D^{k-2}}: n l_{D^{k-3} \rightarrow M_{2}}(X) \neq 1\right\} \cup \xi_{M_{2}}
$$

A inclusão $\supseteq$ é clara, pois se $X \in \mathcal{K}_{M_{3}}$ e $\boldsymbol{M}_{M_{1} \rightarrow M_{2}}(X)=0$ então $n l_{D^{i} \rightarrow M_{2}}(X)=0$ para todo $j=0,1, \ldots, k$.

Passemos a verificar a inclusão contrária. Seja $X \in \xi_{D k-1}$. Os casos não triviais ocorrem quando $X \in \xi_{D^{k-2}}-\xi_{M_{3}}$ e $n l_{D^{k-2} \rightarrow M_{2}}(X)=0$ ou 2. Se $n l_{D^{h-2} \rightarrow M_{2}}(X)=2=\operatorname{gr}\left(D^{k-2} \rightarrow M_{2}\right)$ então $X \in \mathcal{M}\left(D^{k-2} \rightarrow M_{2}\right) \subseteq I_{M_{2}}$. De outro lado, tome $X \in F_{D^{k-2}}-\mathcal{F}_{M_{2}}$ tal que $n l_{D^{k-2} \rightarrow M_{2}}(X)=0$ e mostremos que $n l_{M_{1} \rightarrow M_{2}}(X)=0$. Suponha por absurdo que $n l_{M_{1} \rightarrow M_{3}}(X)>0$. Como $\rho M_{1}(X)>$ $\rho_{M_{2}}(X)=\rho_{D^{k-2}}$ é fácil ver que existe $0<j \leq k-2$ tal que $\rho_{D^{j-1}}(X)=\rho_{D^{j}}(X)+1$ ou equivalentemente, $X \in M\left(D^{j-1} \rightarrow M_{2}\right) \subseteq \xi_{M_{2}}$ já que $D^{j}=N / p$ onde $N$ é a extensão pontual de $D^{j-1}$ pelo filtro modular $\mathcal{M}\left(D^{j-1} \rightarrow M_{3}\right)$. Mas isso é um 
absurdo, portanto $n l_{M_{1} \rightarrow M_{2}}(X)=0$. Concluimos então que em todos of casos possiveis, $X \in \mathcal{I}_{M_{2}}$ ou $n l_{M_{1} \rightarrow M_{2}}(X)=0$.

O levantamento de $M_{2}$ em direção a $M_{1}$ é o marróide $\mathcal{L}\left(M_{1} \rightarrow M_{2}\right)$ sobre $\vec{E}$ com o conjunto de fechados igual a

$$
\left\{X \in I_{M_{1}}: n l_{M_{1} \rightarrow M_{2}}(X)=0\right\} \cup \xi_{M_{2}}
$$

A segaência de levantamentos estritos de $M_{2}$ até $M_{1}$ é a sequência

$$
\begin{aligned}
L^{0}\left(M_{1} \rightarrow M_{3}\right) & =M_{2} \\
L^{i}\left(M_{1} \rightarrow M_{2}\right) & =\mathcal{L}\left(M_{1} \rightarrow L^{i-1}\left(M_{1} \rightarrow M_{2}\right)\right), \quad 1 \leq i \leq \operatorname{gr}\left(M_{1} \rightarrow M_{2}\right)
\end{aligned}
$$

A Proposiça anterior mostra que para todo $j=0,1, \ldots, g r\left(M_{1} \rightarrow M_{2}\right)=$ $k, D^{j}=L^{k-j}$. Note que se $i \geq \operatorname{gr}\left(M_{1} \rightarrow M_{2}\right)$ então $L^{i}\left(M_{1} \rightarrow M_{2}\right)=M_{1}$.

Ábaixo, caracterizamos as sequências de rebaixamentos estritos relacionando alguns dos filtros modulares determinados por esses rebaixamentos.

Proposição 8.4. Seja $Q_{0}, Q_{1}, \ldots, Q_{k}$ uma sequência de matróides sobre $E$ onde $Q_{j}$ é quociente elementar de $Q_{j-1}$ para $j=1,2, \ldots, k$. Então são equivalentes:

8. $Q_{0}, Q_{1}, \ldots, Q_{k}$ é a sequência de rebaixamentos estritos de $Q_{0}$ até $Q_{k}$

ii. $M\left(Q_{j-1} \rightarrow Q_{j}\right) \subseteq M\left(Q_{j} \rightarrow Q_{j+1}\right)$ para todo $j=1, z_{j}, \ldots, k-1$

isi. $M\left(Q_{j-1} \rightarrow Q_{j}\right)=M\left(Q_{j-1} \rightarrow Q_{j+1}\right)$ para todo $j=1,2, \ldots, k-1$

iv. $M\left(Q_{j-1} \rightarrow Q_{j}\right)=M\left(Q_{j-1} \rightarrow Q_{k}\right)$ para todo $j=1,2, \ldots, k$

Prova: A equivalência ii. $\Leftrightarrow$ ìi. segue imediatamente do seguinte fato

$$
M\left(Q_{j-1} \rightarrow Q_{j+1}\right)=M\left(Q_{j-1} \rightarrow Q_{j}\right) \cap M\left(Q_{j} \rightarrow Q_{j+1}\right) \quad j=1,2, \ldots, k-1
$$

Também, a partir desse mesmo fato, obtemos

$$
\begin{aligned}
& M\left(Q_{j-1} \rightarrow Q_{k}\right)=M\left(Q_{j-1} \rightarrow Q_{j}\right) \cap M\left(Q_{j} \rightarrow Q_{j+1}\right) \cap \\
& M\left(Q_{j+1} \rightarrow Q_{j+2}\right) \cap \ldots \cap M\left(Q_{k-1} \rightarrow Q_{k}\right)
\end{aligned}
$$

donde conclui-se facilmente a equivalência ii. $\Leftrightarrow$ iv.. 
Fixemos $1 \leq j \leq k$. Mostremos primeiro que $i . \Leftrightarrow$ iv.. $\wedge$ inclusão $M\left(Q_{j-1} \rightarrow Q_{j}\right) \supseteq M\left(Q_{j-1} \rightarrow Q_{k}\right)$ é clara. Tome, de outro lado, $X \in M\left(Q_{j-1} \rightarrow\right.$ $\left.Q_{j}\right)$. Lembrando a definição de $M\left(Q_{j-1} \rightarrow Q_{j}\right)$ temos

$$
\rho_{Q_{j}}(X)=\rho_{Q_{j-1}}(X)-1
$$

Considere agora a extensão pontual $N$ de $Q_{j-1}$ sobre $E \cup p$ pelo filtro modular $M\left(Q_{j-1} \rightarrow Q_{k}\right)$. Assim, $N / p=D^{j}\left(Q_{0} \rightarrow Q_{k}\right)=Q_{j}$ e portanto

$$
\rho_{Q_{j}}(X)=\rho_{N}(X \cup p)-\rho_{N}(p)=\rho_{N}(X \cup p)-1
$$

Combinando $(I)$ e $(I I)$, segue que $p \in \bar{X}^{N}$, isto $\varepsilon, X \in M\left(Q_{j-1} \rightarrow Q_{k}\right)$. Finalmente passemos a verificar que $i v . \Rightarrow$ i.. A igualdade $M\left(Q_{j-1} \rightarrow Q_{j}\right)=M\left(Q_{j-1} \rightarrow\right.$ $\left.Q_{k}\right)$ implica que $N(E \cup p)=N^{\prime}(E \cup p)$ onde $N^{\prime}$ é a extensão pontual de $Q_{j-1}$ sobre EU p pelo filtro modular $M\left(Q_{j-1} \rightarrow Q_{j}\right)$. Mas então,

$$
D^{i}\left(Q_{j} \rightarrow Q_{k}\right)=N / p=N^{\prime} / p=Q_{j}(E)
$$

Finalizamos apresentando dois resultados que serão úteis na próxima seção. Neles tentamos relacionar a sequência de rebaixamentos estritos de $M_{1}$ até $M_{3}$ com à sequência de rebaixamentos estritos de $M_{2}$ até o mesmo $M_{3}$, onde $M_{3}$ é quociente de $M_{2}$ e $M_{2}$ por sua ver é quociente elementar de $M_{1}$.

Lema 8.5. Sejam $M_{1}, M_{2}, M_{3}$ e $M_{4}$ matróides sobre $E$ tajs que $M_{2}$ é quociente de $M_{3}$ e $M_{4}$, esses dois últimos são quocientes de $M_{1}$ e gr $\left(M_{1} \rightarrow M_{4}\right)=\operatorname{gr}\left(M_{3} \rightarrow\right.$ $\left.M_{2}\right)=1$. Se $M\left(M_{1} \rightarrow M_{3}\right) \cap M\left(M_{1} \rightarrow M_{4}\right)=M\left(M_{1} \rightarrow M_{2}\right)$ então $D\left(M_{4} \rightarrow M_{2}\right)$ é quociente elementar de $D\left(M_{1} \rightarrow M_{3}\right)$.

Prova: Para não carregar a notação usaremos $M(i \rightarrow j), n l_{i \rightarrow j}, D(i \rightarrow j)$, gr $(i \rightarrow$ j) e $\rho_{i}$ ao invés de $M\left(M_{i} \rightarrow M_{j}\right), n l_{M_{i} \rightarrow M_{j}} D\left(M_{i} \rightarrow M_{j}\right), \operatorname{gr}\left(M_{i} \rightarrow M_{j}\right)$ e $\rho_{M_{i}}$, respectivamente.

Fixemos $X \in D(4 \rightarrow 2)$. Se $X \in M(4 \rightarrow 2)$ então $X \in M_{2} \subseteq D(1 \rightarrow 3)$. Suponhamos então que $X \notin M(4 \rightarrow 2)$. Assim,

$$
n l_{4 \rightarrow 2}(X) \leq \operatorname{gr}(4 \rightarrow 2)-2
$$


Examinemos dois casos possiveis:

Caso 1: $X \notin M(1 \rightarrow 4)$, isto é, $n l_{1 \rightarrow 4}(X)=0$

Temos que

$$
\begin{aligned}
n l_{1 \rightarrow 3}(X) & \leq n l_{1 \rightarrow 2}(X)=n l_{1 \rightarrow 4}(X)+n l_{1 \rightarrow 2}(X) \\
& (I) g r(4 \rightarrow 2)-2
\end{aligned}
$$

Mas $\operatorname{gr}(4 \rightarrow 3)=\operatorname{gr}(1 \rightarrow 3)$; denotemos esse número por n. Logo, $n l_{1 \rightarrow 3}(X)<\operatorname{gr}(1 \rightarrow 3)-1$, o que mostra que $X \in D(1 \rightarrow 3)$.

Caso 2: $X \in M(1 \rightarrow 4)$, isto é, $n l_{1 \rightarrow 4}(X)=1$

Pela Proposição 2.3., $M(1 \rightarrow 2)=M(1 \rightarrow 4) \cap M(4 \rightarrow 2)$ e como $X \notin$ $M(4 \rightarrow 2)$ temos que $X \notin M(1 \rightarrow 2)$. Por outro lado, por hipbtese, $M(1 \rightarrow$ $2)=M(1 \rightarrow 3) \cap M(1 \rightarrow 4)$, donde $X \notin M(1 \rightarrow 3)$, ou equivalentemente, $n l_{1 \rightarrow 3}(X) \leq n-1$. Suponha por absurdo que $X \notin M(1 \rightarrow 3)$. Então existe $Y \in M(1 \rightarrow 3)$ tal que $Y$ cobre $X$. Como $X \in M(1 \rightarrow 4)$ temos também que $Y \in M(1 \rightarrow 4)$, e usando novamente a igualdade $M(1 \rightarrow 2)=M(1 \rightarrow$ 3) $\cap M(1 \rightarrow 4)$ resulta que $Y \in M(1 \rightarrow 2)$, isto é, $n l_{1 \rightarrow 2}(Y)=n+1$. Daf obtemos

$$
\begin{aligned}
n l_{1 \rightarrow 2}(X) & =\rho_{1}(X)-\rho_{2}(X) \\
& \geq \rho_{1}(Y)-\rho_{2}(Y)-1=n
\end{aligned}
$$

Mas

$$
\begin{gathered}
n l_{1 \rightarrow 2}(X)=n l_{1 \rightarrow 4}(X)+n l_{4 \rightarrow 2}(X) \\
\text { (I) } \\
\leq n-1
\end{gathered}
$$

uma contradição. Portanto, $X \in D(1 \rightarrow 3)$.

Concluímos então que $D(4 \rightarrow 2)$ é quociente de $D(1 \rightarrow 3)$ e em particular,

$$
\begin{aligned}
\operatorname{gr}(D(1 \rightarrow 3) \rightarrow D(4 \rightarrow 2)) & =\rho D(1 \rightarrow 3)-\rho D(4 \rightarrow 2) \\
& =\left(\rho M_{1}+1\right)-\left(\rho M_{4}+1\right) \\
& =\operatorname{gr}(1 \rightarrow 4)=1
\end{aligned}
$$


Proposição 8.6. Sejam $M_{1}, M_{2}$ e $M_{3}$ matróides sobre $E$ tais que $M_{3}$ é quociente de $M_{2}, M_{2}$ é quociente de $M_{1}$ e $\operatorname{gr}\left(M_{1} \rightarrow M_{2}\right)=1$. Então $D^{k}\left(M_{2} \rightarrow M_{3}\right)$ é quociente elementar de $D^{k}\left(M_{1} \rightarrow M_{3}\right)$ para $k=0,1, \ldots, g r\left(M_{2} \rightarrow M_{3}\right)$.

Prova: Por indução sobre k. Como $D^{0}\left(M_{2} \rightarrow M_{3}\right)=M_{2}$ e $D^{0}\left(M_{1} \rightarrow M_{3}\right)=M_{1}$, por hipótese $D^{0}\left(M_{2} \rightarrow M_{3}\right)$ é quociente elementar de $D^{0}\left(M_{1} \rightarrow M_{3}\right)$.

Suponha agora que $k>0$. Por hipótese de indução, $D^{k-1}\left(M_{2} \rightarrow M_{3}\right)$ é quociente elementar de $D^{k-1}\left(M_{1} \rightarrow M_{3}\right)$. Temos então o diagrama a seguir, onde $A \rightarrow B$ quer dizer que o matróde $B$ é quociente do matróide $A$.

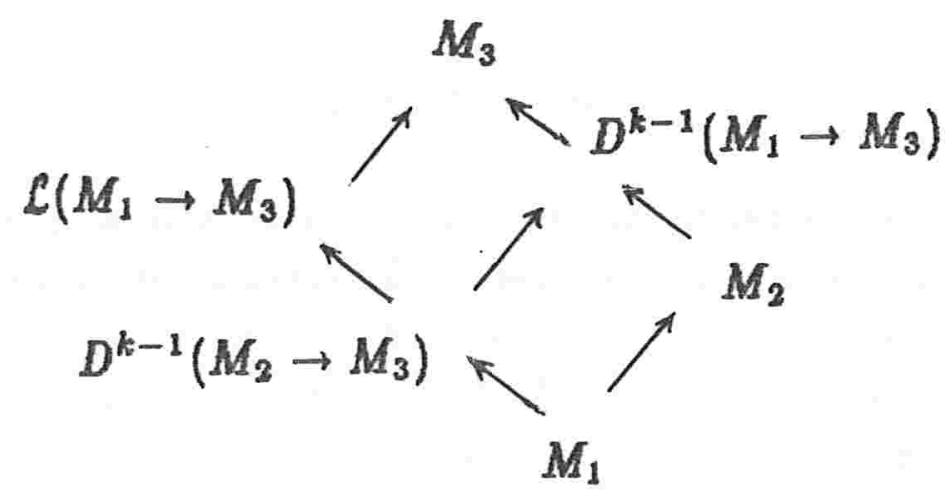

Mostremos que

$$
\begin{gathered}
M\left(D^{k-1}\left(M_{1} \rightarrow M_{3}\right) \rightarrow \mathcal{L}\left(M_{1} \rightarrow M_{3}\right)\right) \cap M\left(D^{k-1}\left(M_{1} \rightarrow M_{3}\right) \rightarrow\right. \\
\left.D^{k-1}\left(M_{2} \rightarrow M_{3}\right)\right)=M\left(D^{k-1}\left(M_{1} \rightarrow M_{3}\right) \rightarrow M_{3}\right)
\end{gathered}
$$

pois nesse caso estaremos nas hipóteses do Lema anterior em relação a $D^{k-1}\left(M_{1} \rightarrow\right.$ $\left.M_{3}\right), M_{3}, \mathcal{L}\left(M_{1} \rightarrow M_{3}\right), D^{k-1}\left(M_{2} \rightarrow M_{3}\right)$ e portanto $D\left(D^{k-1}\left(M_{2} \rightarrow M_{3}\right) \rightarrow\right.$ $\left.M_{3}\right)=D^{k}\left(M_{2} \rightarrow M_{3}\right)$ é quociente elementar de $D\left(D^{k-1}\left(M_{1} \rightarrow M_{3}\right) \rightarrow \mathcal{L}\left(M_{1} \rightarrow\right.\right.$ $\left.\left.M_{3}\right)\right)=D^{k}\left(M_{1} \rightarrow M_{3}\right)$.

A inclusão $\supseteq$ é facilmente verificável recorrendo-se à Proposição 3.4.. A inclusão contrária segue do seguinte fato, também consequência da mesma proposição

$$
\mathcal{M}\left(D^{k-1}\left(M_{1} \rightarrow M_{3}\right) \rightarrow \mathcal{L}\left(M_{1} \rightarrow M_{3}\right)\right) \subseteq \mathcal{M}\left(D^{k-1}\left(M_{1} \rightarrow M_{3}\right) \rightarrow M_{3}\right)
$$

\section{REPRESENTACÕES DE QUOCIENTES}

Nesta seção, não pretendemos nos aprofundar na teoria da representabilidade de quocientes. Gostaríamos apenas de introduzf-la, apresentar sua ligação 
com a teoria da compatibilidade de extensôes e, quem sabe, motivar o leitor a prosseguir num estudo mais aprofundado.

Seja $I$ um subconjunto finito dos naturais. Uma representasano de uma familia $\left\{Q_{i}\right\}_{i \in I}$ de quocientes de $M$ é uma extensão $N$ de $M$ sobre $E \cup P$ juntamente com uma familia $\left\{Y_{i}\right\}_{i \in I}$ de fechados de $N(P)$ tal que para todo $i \in I$, $Q_{i}\left(N / Y_{i}\right)(E)$. Pelo Teorema 2.4. vale a seguinte igualdade

$$
\rho_{N}\left(X \cup Y_{i}\right)=\rho_{Q_{i}}(X)+\rho_{N}\left(Y_{i}\right)
$$

para todos $i \in I$ e $X \subseteq E$. Vale também que

$$
\operatorname{gr}\left(M \rightarrow Q_{i}\right) \leq \rho_{N}\left(Y_{i}\right)
$$

Se para todo $i \in I$ ocorrer igualdade na expressz̃o acima, a representação é dita estrita. Se $\left\{Q_{i}\right\}_{i \in I}$ tem uma representação (resp., representação estrita) então digemos que $\left\{Q_{i}\right\}_{i \in I}$ são representáveis (resp., estritamente representáveis).

A Proposição 2.10. do Capitulo II mostra que qualquer quociente de um matróide fem uma representação estrita. Esse resultado foi inicialmente provado por Higgs em [Hi]. A partir da teoria sobre quocientes desenvolvida nesse capftulo, esse resultado pode ser facilmente obtido. De fato, sejam $M^{\prime}$ un quociente de $M$ e $R$ um matróide sobre $P$ tal que $p R=\operatorname{gr}\left(M \rightarrow M^{\prime}\right)$. A famlia $\{Q(Y)\}_{Y \in F_{R}}$ definida por

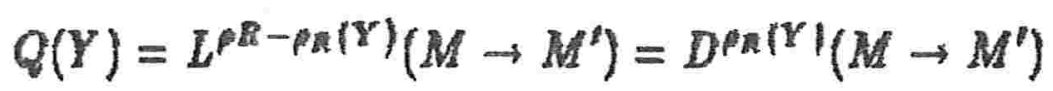

é um $R$-feixe estrito de $M$. Logo, a extenzão $N$ de $M$ sobre $E \cup P$ tal que $N(P)=R$ e $Q(Y)=(N / Y)(E)$ para todo $Y \in \mathscr{I}_{R}$ é uma representação estrita de $M^{\prime}$.

O natural agora é investigarmos a representabilidade de vários quocientes de $M$. Enquanto o problema geral de representação permanece em aberto, existem resultados positivos relacionados com representaçōes de quocientes elementares de M. Em particular, é possivel caracterizar uma famllia de quocientes elementares de $M$ estritamente representáveis, ligando os conceitos de representabilidade de quocientes e compatibilidade de filtros modulares como mostra o resultado seguinte:

Proposiço 4.1. Sejam $Q_{1}, Q_{3}, \ldots, Q_{n}$ quocientes elementares de $M$. Temos então que $Q_{1}, Q_{2}, \ldots, Q_{n}$ são estritamente representáveis se e somente se $M(M \rightarrow$ $\left.Q_{1}\right), M\left(M \rightarrow Q_{2}\right), \ldots, M\left(M \rightarrow Q_{n}\right)$ são filtros modulares compativeis.

Prova: Omitimos, simples manipulação de definições. 
Assim é possível que exista uma familia $\left\{Q_{i}\right\}_{i \in I}$ de quocientes elementares de $M$ representável mas não estritamente representável. Exemplos desse fato podem ser obtidos com pares de quocientes elementares, bastando combinar a Proposição 4.1., o Teorema 4.2. a seguir, e o fato de existirem pares de filtros modulares não compativeis.

Teorema 4.2. Sejam $Q_{1}, Q_{2}$ quocientes elementares de $M$. Então o par $Q_{1}, Q_{2}$ é representável.

Prova: Escolha $Q_{12}$ um quociente comum de $Q_{1}, Q_{2}$ e denote por $n$ o gr $(M \rightarrow$ $\left.Q_{12}\right)$. Seja $R=\mathcal{L}_{2 n-1}(A \cup B)$ onde $\mathbb{A}=\left\{a_{1}, a_{2}, \ldots, a_{n}\right\}, B=\left\{b_{1}, b_{2}, \ldots, b_{n}\right\}$ e $A \cap B=\emptyset=(A \cup B) \cap E$. Para cada $Y \in \mathcal{F}_{R}$ definimos

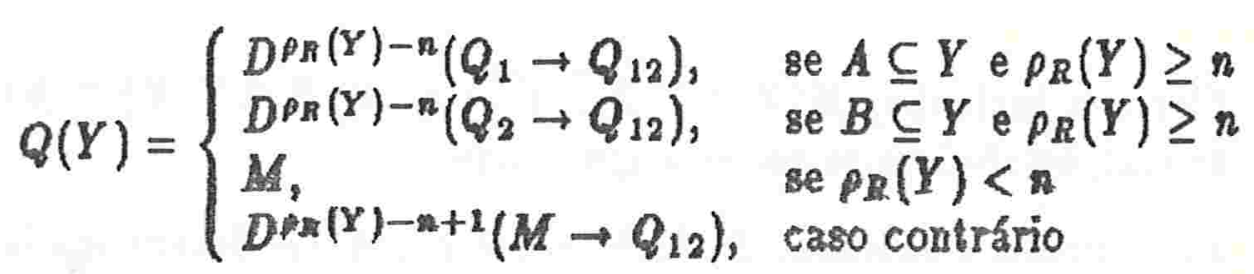

Claramente $Q(A)=Q_{1}$ e $Q(B)=Q_{2}$. Afrmamos que $\left\{Q_{Y}\right\}_{Y \in \mathcal{F}_{B}}$ é um R-feixe sobre $M$. De fato, as propriedades (Q1), (Q2), (Q3) da Proposição 2.1. estão satisfeitas:

(Q1) $Q(0)=M$ trivialmente pois $n>0$

(Q2) Sejam $X, Y \in \xi_{R}$ tais que $Y$ cobre $X$. t bvio que se $A \subseteq X$ ou $B \subseteq X$ ou $A, B \nsubseteq Y, Q(Y)$ é quociente de $Q(X)$ e $\operatorname{gr}(Q(X) \rightarrow Q(Y)) \leq 1$. 0 único caso não trivial ocorre quando nem $A$ e nem $B$ são subconjuntos de $X$, e $A$ ou $B$ está contido em $Y$. Suponha que $A \subseteq Y, \circ$ caso $B \subseteq Y$ é análogo. Se $\rho_{R}(X)<n$ então $Y=A$ e portanto $Q(Y)=Q_{1}$ é quociente elementar de $Q(X)=M$. Se $\rho_{R}(X) \geq n$ então $Q(X)=D^{\rho_{R}(X)-n+1}\left(M \rightarrow Q_{12}\right)=$ $D^{p_{R}(Y)-n}\left(M \rightarrow Q_{12}\right)$ e $Q(Y)=D^{\rho_{R}(Y)-n}\left(Q_{1} \rightarrow Q_{12}\right)$, portanto pela Proposição 3.6., $Q(Y)$ é quociente elementar de $Q(X)$.

(Q3) Sejam $X, Y \in \xi_{R}$ tais que $X, Y$ cobrem $X \cap Y$. Inicialmente observamos que não é possivel que $A \subseteq X$ e $B \subseteq Y$ simultaneamente. De fato, se isso acontecesse, $X \cup Y=A \cup B$ e portanto $X, Y$ seriam hiperplanos de $R$, uma contradição já que $\rho(X \cap Y)=|X \cap Y|=|X|+|Y|-|X \cap Y|=2 n-4$, isto é, $X \cap Y$ não é coplano de $R$. Da mesma forma concluímos que não podemos ter $B \subseteq X$ e $A \subseteq Y$. Se $A \subseteq X \cap Y$ (resp., $B \subseteq X \cap Y$ ) então $Q(X \cap Y), Q(X), Q(Y)$ e $Q\left(\overline{X \cup Y}^{R}\right)$ são rebaixamentos de $Q_{1}$ (resp., 
$Q_{2}$ ) em direção a $Q_{12}$. Usando o item i̊i. da Proposição 3.4. obtemos a igualdade

$$
M(X \cap Y, X) \cap M(X \cap Y, Y)=M\left(X \cap Y, \overline{X \cup Y}^{\mathbb{R}}\right)
$$

So restam dois casos a considerar: $A, B \nsubseteq X$ ou $A, B \nsubseteq Y$. Suponha que $A, B \nsubseteq X$. Nesse caso $Q(X \cap Y), Q(X)$ são rebaixamentos de $M \mathrm{em}$ direção a $Q_{12}$ e pelo item iv. da Proposição 3.4. temos que $M(X \cap Y, X)=$ $M(X \cap Y, A \cup B)$. Logo,

$$
\begin{aligned}
M(X \cap Y, X) \cap M(X \cap Y, Y) & \subseteq M(X \cap Y, X) \\
& =M(X \cap Y, A \cup B) \\
& \subseteq M\left(X \cap Y, \overline{X \cup Y}^{\mathbb{Z}}\right)
\end{aligned}
$$

Como a inclusão, $M\left(X \cap Y, \overline{X \cup Y}^{R}\right) \subseteq M(X \cap Y, X) \cap M(X \cap Y, Y)$ é trivial, estabelece-se assim a igualdade.

Finalmente, a extensão $N$ de $M$ sobre $E \cup A \cup B$ determinada pelo $R$-feixe $\{Q(Y)\}_{Y \in I_{R}}$ é uma representação de $Q_{1}, Q_{2}$, já que $N / A(E)=Q(A)=Q_{1}$ e $N / B(E)=Q(B)=Q_{2}$.

A técnica da demonstração do teorema anterior consistiu basicamente em encontrar um matróide $R$ conveniente e completar o par de quocientes $Q_{1}, Q_{2}$ a um R-feixe de quocientes de $M$. Essa é uma maneira interessante de encarar o problema da representabilidade e, nessa linha, Cheung em [Ch] estuda os feires parciass de quocientes que são famílias de quocientes de um matróide $M$ indexadas pelos fechados de um outro matróide $R$ sobre $P$, e que podem ser completados a um $R$-feixe de $M$. Cheung fixa-se principalmente nas várias formas possíveis em que um feixe parcial pode ser completado a um $\mathrm{R}$-feixe de $M$. 


\section{Referências Bibliográficas}

[Ai] M. Aigner, "Combinatorial Theory", Springer - Verlag, Berlin/ Heidelberg/New York (1979).

[Ch] A.L.C. Cheung, Compatibility of Extensions of a Combinatorial Geometry, Thesis, Univ. of Waterloo, Ontario (1974).

[Co1] R. Cordovil, Extensions Lisses d'une Géométrie Combinatoire, C.R. Acad. Sci. Paris Sér. A 284 (1977), 1249-1252.

[Co2] R. Cordovil, Sur la Compatibilité des Extensions Ponctuelles d'un Matroide, J. Comb. Theory Ser. B 34 (1983), 209-223.

[Cr] H. Crapo, Single Element Extensions of Matroids J. Res. Nat. Bur. Stand. Sect. B 69 (1965), 57-65.

[Hi] D.A. Higgs, Strong Maps of Geometries, J. Comb. Theory 5 (1968), 185-191.

[LV1] M. Las Vergnas, Extensions Normales d'un Matroïde, Polynôme de Tutte d'un Morphisme, C.R. Acad. Sci. Paris Sér. A 280 (1975), 1479-1482.

[LV2] M. Las Vergnas, Extensions Ponctuelles Compatibles d'une Gc. ometrie Combinatoire, C.R. Acad. Sci. Paris Sér. A 286 (1978), 981-983. 
[Ma] J.H. Mason, Matroids as the study of Geometrical Configurations, "Higher Combinatorics", M.Aigner ed. (1977), 133-176.

[vW] B.L. van der Waerden, "Moderne Algebra", Springer, Berlin, 2 ed. (1973).

[We] D.H. Welsh, "Matroid Theory", Academic Press, New York/S. Francisco/London (1976).

[Wh] H. Whitney, On the Abstract Properties of Linear Dependence, Amer. J. Math. 57 (1935), 509-533. 
Extensão, 8

- lisa, 22

- pontual, 8

F

Fechado, 3

Fecho, 3

- de filtro modular, 14

- de subclasse linear, 72

Feixe

- de guocientes, 80

- parcial, 96

Filtros

- 2-compativeis, 55

- modulares, 0

- modulares gerados, 9

- modulares associados a subclasses lineares, 71

- principais, 9

- ultra-compatíveis, 15

f-nulidade, 10

Funçz̃o altura, 5

G

Grau

- de um empilhamento, 34

- de um quociente, 10
H

Hiperplano, 3

I

Independente, 2

- afim, 3

L

Laço, 3

Levantamento, 90

Linha, 3

$\mathbf{M}$

Mais livre, 7

Matroide, 2

- aîm, 3

- grá́ić, 3

- linear, 2

- livre, 2

- iransversal, 3

- uniforme, 2

Morísmo forte, 10 


\section{Indice Remissivo}

f̂ltros modulares,- 9

subclasses lineares $=, 72$

Contração, 7

Coplano, 3

Coponto, 3

D

Defeito, 10

Dependente, 3

2-extensäo, 9

Base, 3

C

Circuitos, 3

Colinha, 3

Compativeis
E

Elementos paralelos, 8

Empilhamento, 34

- de una extensão, 30 - de um flltro modular 2-compatível, $\$ 5$ 
$\mathbf{N}$

Normal, 82

conjunto, 32

extensล๊o, 33

Nulidade, 10

(1)

Ordem fraca, 8

Par modular, 9

Plano, 3

Ponto, 3

Posto, 3

Quocientes, 10

- elementares, 11

- representáveis, 94

- esfritamente

representsveis, 94
$\mathbf{R}$

Rebaixamento, 88

Representação, 94

- estrita, 94

Restriçz̃o, 7

Reticulado

- de um matróide, 5

- geométrico, 6

- graduado, 5

- semimodular, 6

R-\{eixe, 85

- estrito, 85

$\mathbf{S}$

Sequê̂ncias

- de levantamentos estritos, 90

- de rebaixamentos estritos, 89

- geradoras, 71

- ultra-compatíveis, 15

Subclasse linear, 70

- associada a um filtro

modular, 71

Soma de um ponto, 80

$\mathrm{T}$

Tripla geradora, 70

Truncamento, 6 


\section{DOACAO}

DATA: 19.08 .87

BIBLIOTECA "CARI OS BEN JAMINI DE LYRA" INSTITUTO DE MAIEMÁTICA E ESTATISTICA - unIVERSIDAde ue sá PAUlo Cidade Universitária C.P.66.281 - AG. Cidade de São P.aulo 05389-970 - SAO PAULO - BRASIL Tel: $818.6174 ; 818.6109 ; 818.6269 \mathrm{Fax}: 818.5036$ e-mall: mb@ime.usp.br 


\section{Errata}

\begin{tabular}{|c|c|c|c|}
\hline Pág. & Linhe & Berito & Corrigido \\
\hline xviii & $7 \downarrow$ & fig.11.a & fig.12.a \\
\hline 17 & $7 !$ & $\{1,2, \ldots, n-1\}$ & $\{1,2, \ldots, n\}$ \\
\hline 18 & $4 !$ & tais que & $e|f|=d+1$ tal que \\
\hline 20 & $2 \uparrow$ & Teorema 1.7. & Teorema 1.8 \\
\hline 24 & $2 \uparrow$ & $\rho_{N}\left(\bar{X}^{N}\right)-$ & $\bar{X}^{N}-$ \\
\hline 24 & $8 \uparrow$ & $\left.-\bar{z}^{N} \mid\right\}$ & $\left.-\overline{\mathbb{Z}}^{N} \mid, X \cap E \subseteq \mathbb{Z}\right\}$ \\
\hline $\begin{array}{l}27 \\
27\end{array}$ & $\begin{array}{l}1 \uparrow \\
3 \uparrow\end{array}$ & $\begin{array}{l}x \in \bar{X}^{M^{\prime}} \cap X \\
\text { vitura-compativeis }\end{array}$ & $x \in X$ ou $x \in \bar{X}^{M^{\prime}} \cap E$ \\
\hline 28 & 11 & $z \in \bar{X}^{M^{\prime}}-X$ & $z \in\left(\bar{X}^{M^{\prime}}-X\right) \cap F$ \\
\hline 28 & $2 !$ & $\in \bar{I}_{i}$ & $\begin{array}{l}\in \mathcal{F}_{i} \text { para algum } \\
i \in\{1,2, \ldots, n\}\end{array}$ \\
\hline 31 & $2 \uparrow$ & formam & forma \\
\hline 32 & $13 \downarrow$ & $M(X \cup A) \backslash X$ & $M(X \cup A) / X$ \\
\hline 33 & $4,5,6 \downarrow$ & $\begin{array}{l}\text {, o que mostra...que } \\
\rho(M(A))_{k}=\end{array}$ & \\
\hline & & $\rho M(X \cup A) / X$ & \\
\hline 33 & $11 \downarrow$ & $\overline{X \cup Y^{M}}-X=A$ & $\overline{X \cup Y_{0}}-X \supseteq A$ \\
\hline 33 & $12 !$ & $\rho_{M / X}\left(Y_{0}\right)=\rho_{M / X} A$ & $\rho_{M} / X\left(Y_{0}\right) \geq \rho_{\mathbb{M} / X}(A)$ \\
\hline 33 & $14 !$ & $\rho_{M}(X \cup A) / X$ & $\begin{array}{l}\rho_{M}(X \cup A) / X \text {. Tome } B \\
\text { base de } M(X \cup A) / X \text {. } \\
\text { É claro que } B \subseteq A, \\
|B|=k \text { e } B \cup X \text { é } \\
\text { independente em } M . \\
\text { Logo } B \text { é independente } \\
\text { em } M(A) \text { e portanto } \\
B \text { é base de }(M(A))_{k} \text {. }\end{array}$ \\
\hline 35 & $1 \uparrow$ & $i \neq j$ & $i \neq k$ \\
\hline 38 & 81 & +1 & -1 \\
\hline 38 & $\theta \uparrow$ & +2 & -2 \\
\hline 38 & $11 \uparrow$ & +2 & -2 \\
\hline 39 & $4 !$ & $\rho N$ & $\rho_{N}(P)$ \\
\hline 39 & $7 \downarrow$ & $\{a, b, c, d\}$ & $\{a, b, c, d, e\}$ \\
\hline 40 & $13 \uparrow$ & (R1) & (R2) \\
\hline 41 & 11 & $i+1$ & $\mid i-1$ \\
\hline
\end{tabular}




\begin{tabular}{|c|c|c|c|}
\hline 48 & 41 & $j+i)$ & $j+1)$ \\
\hline 42 & $4 \uparrow$ & e portanto $w \notin \overline{X \cup x}^{M}$ & \\
\hline 43 & $6 \uparrow$ & $=p_{M}(X)+i$ & $\begin{array}{l}=\rho_{M}(X)+i . \text { Se } w \in \overline{X \cup z} \\
\text { entiúto } \\
\rho_{N}(X \cup Y \cup z \cup w)=\rho_{N}(X \cup Y) \\
\text { trivialmente. } \\
\text { Suponha que } w \notin \overline{X \cup z} \text {. } \\
\text { nesta seção }\end{array}$ \\
\hline 48 & $7 !$ & $P_{M}$ & $P N$ \\
\hline 48 & $15 \uparrow$ & $\pi_{1} \Delta \pi_{2} \subseteq$ & $\xi_{1} \cup \xi_{2} \subseteq \varepsilon^{(0)} \cup$ \\
\hline 48 & $16 \uparrow$ & $X \in \varepsilon^{(0)}$ & $X \notin E(0)$ \\
\hline 40 & 11 & $\xi_{1} \subseteq \xi_{2}$ & $\xi_{1} \cap \xi_{2}$ \\
\hline 49 & 51 & Proposição 1.8. & Proposição 2.8. \\
\hline 49 & $13 \downarrow$ & $P_{M}(X)$ & $p_{M}(X)+1$ \\
\hline 53 & $1 \uparrow$ & $i_{i} \Rightarrow i v$ & $i . \leftarrow i v$ \\
\hline 53 & $1 \uparrow$ & isi. $\Rightarrow$ si. & iii. $\Leftarrow$ ii. \\
\hline 54 & $2 \uparrow$ & $\Gamma\left(\xi_{1}, \xi_{2}\right)$ & $\Phi\left(\xi_{1}, \xi_{2}\right)$ \\
\hline 54 & $7 \uparrow$ & $\bar{X}^{N \prime} \in \xi_{2}^{\prime}$. & $\bar{X}^{N_{1}} \in \xi_{2}^{\prime}$ \\
\hline 57 & $2 \uparrow \ldots$ & e usando iv.(b) & nsando iv.(b), \\
\hline 57 & $3 \uparrow$ & $X \in \mathcal{E}_{1}^{(8)}$ & $Y \in \mathcal{E}_{1}^{(i)}$ \\
\hline 59 & $6 !$ & $\leq \rho_{N}(Z)+1$ & $=P_{N}(Z)+1$ \\
\hline 65 & $7 !$ & dos seguintes fatos & do seguinte fato \\
\hline 65 & & se existe... $\left(\bigcap_{j \in I} \xi_{j}\right)$ & \\
\hline 70 & $6,7,8,8,10,11 \uparrow$ & $\begin{array}{l}\text { Por definição... iltro } \\
\text { modular de } M \text {. }\end{array}$ & A demonstração pode \\
\hline 71 & $5 \uparrow$ & $A \in X$ & $A \subseteq X$ \\
\hline 73 & $4 !$ & $S_{1, i}^{i}=$ & $S_{1}^{i}$, para algum $i=$ \\
\hline 73 & $5 !$ & $\mathfrak{S}_{3}^{i}, i=$ & $S_{2}^{i}$, para algum $i=$ \\
\hline 73 & $15 !$ & então Z & então $\mathbb{Z} \in \mathcal{I}_{M}$ \\
\hline 73 & $16,17 \downarrow$ & $Z \notin X_{\text {....contradição }}$ & $\begin{array}{l}Z \in X \text { se e somente se } \\
Z \in S_{1}-S_{2} \text {. Suponha então } \\
\text { que } p M(Z)<\rho M-1 .\end{array}$ \\
\hline 73 & $18 !$ & & $\begin{array}{l}\text { não coberta por elemento } \\
\text { de } \xi_{1}\end{array}$ \\
\hline 75 & $2 \downarrow$ & $\left.S_{3}^{i}\right\} \subseteq S_{2}$ & $\begin{array}{l}S_{2}^{i}, \text { para algum } \\
i=0,1, \ldots\} \subseteq S_{2}\end{array}$ \\
\hline 75 & $2 \downarrow$ & $S_{2}^{i}, i=$ & $S_{2}^{i}$, para algum $i=$ \\
\hline 78 & $8 \uparrow$ & $\overline{X \cup x^{M}}$ & $\overline{X \cup x}$ \\
\hline 79 & $5 \downarrow$ & $\rho \mathbf{M}_{3}(X)$ & $\rho_{\mathbf{M}_{2}}(H)$ \\
\hline
\end{tabular}




\begin{tabular}{|c|c|c|c|}
\hline 81 & 21 & $\begin{array}{l}\text {, isto } \varepsilon, Q_{Y} \text { E } \\
\text {...de } Q_{X}\end{array}$ & \\
\hline 81 & $2 !$ & $z \in Q_{Y}$ & $Z \in Z_{Q x}$ \\
\hline 81 & $5 !$ & $z \in Q_{x}$ & $Z \in Z_{Q_{x}}$ \\
\hline 84 & 21 & $p N$ & $P_{N}$ \\
\hline 85 & $1 \downarrow$ & $\overline{Y \cup z}$ & $\overline{Y \cup w}^{R}$ \\
\hline 85 & $11 \downarrow$ & $\rho N$ & $\rho_{N}$ \\
\hline 86 & $2 \downarrow$ & izi. & iv. \\
\hline 87 & $8 !$ & $\{X$ & $\xi^{*}=\{X$ \\
\hline 87 & $12 !$ & $\xi_{N / P}=\xi$ & $\xi_{N / p}=\xi^{*}$ \\
\hline 87 & $14 !$ & $\xi_{M_{3}}$ & 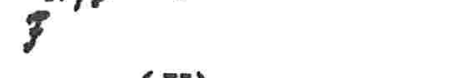 \\
\hline 89 & $3 \uparrow$ & $P_{D L-8}$ & $P_{D^{k-8}}(X)$ \\
\hline 89 & $13 \uparrow$ & $s \in \mathcal{F}_{M_{1}}$ & $X \in \tilde{I}_{M_{1}}$ \\
\hline 91 & $1 \downarrow$ & i. $\Leftrightarrow s v$. & $\dot{i}_{0} \Rightarrow \mathrm{i} v_{\text {. }}$ \\
\hline 91 & $12 \downarrow$ & $D^{j}\left(Q_{j} \rightarrow Q_{k}\right)$ & $D\left(Q_{j-1} \rightarrow Q_{k}\right)$ \\
\hline 91 & $2 \uparrow$ & $M(4 \rightarrow 2)$ & $M(4 \rightarrow 2) \cup \xi_{M_{3}}$ \\
\hline 91 & $3 \uparrow$ & $M_{3} \subseteq D(1 \rightarrow 3)$ & $I_{M_{3}} \subseteq F_{M_{3}} \subseteq F_{D(1-3)}$ \\
\hline 92 & $7 !$ & $D(1 \rightarrow 3)$ & $I_{D(1 \rightarrow 3)}$ \\
\hline 92 & $12 !$ & $M(1 \rightarrow 3)$ & $I_{D(1 \rightarrow 3)}$ \\
\hline 92 & $5 \uparrow$ & $\in D(1 \rightarrow 3)$ & $\in S_{D(1 \rightarrow 3)}$ \\
\hline 93 & 91 & $D^{k-1}\left(M_{2} \rightarrow M_{3}\right)$ & $D^{k-1}\left(M_{1} \rightarrow M_{3}\right)$ \\
\hline 93 & 9! & $D^{k-1}\left(M_{1} \rightarrow M_{3}\right)$ & $D^{k-1}\left(M_{2} \rightarrow M_{3}\right)$ \\
\hline 94 & $6 !$ & $Q_{i}$ & $Q_{i}=$ \\
\hline 95 & $4 \uparrow$ & $|X \cap Y|$ & $|\boldsymbol{X} \cup \boldsymbol{Y}|$ \\
\hline
\end{tabular}


